\title{
Photonic Integrated Circuits for Application in Optical Routing, Computing and Beamforming
}

\author{
Md Abduhu Ruhul Fatin \\ Department of Electrical and Computer Engineering \\ Carleton University, Ottawa \\ December, 2020
}

A thesis submitted to Carleton University in partial fulfillment of the requirements of the degree of

Master of Applied Science

CM. A. R. Fatin, 2020 


\section{Abstract}

Phase of the guided light is a fundamental and important property to control and operate a photonic integrated circuit (PIC). Silicon photonic PICs provide potential for low-cost, scalable solutions to a myriad of applications such as optical logic, optical communications and routing. This thesis presents a theoretical and experimental demonstration of controlling an on-chip optical phased array (OPA) - a photonic device for optical beamforming and beam steering. This thesis also presents a work on optical logic architectures where two different phase control schemes are applied on the same device. Finally, optical routing architectures are proposed that have ultracompact photonic switches as the building block. All these devices fall under the umbrella of programmable PICs and this thesis is focused on the design and control of programmable PICs of various applications.

Robust and efficient controlling of OPAs is an important task to support large-scale integration. In this thesis, we present a method to optimize the performance of multiple concentric ring OPAs through far field simulations. Using the far field simulation approaches we investigate the efficiency and computation cost of gradient descent based beam control. Afterwards, we propose a new closed loop control algorithm and compare them with other control mechanisms. We performed experimentations on an on-chip OPA to verify our simulated results.

PN junctions and phase change materials can be used to tune the phase of guided light. A novel optical device with microdisks has been proposed in this thesis that can be applied on a directed logic architecture. The primary focus of this design was to minimize the number of costly opto-electric conversions in the architecture. The design utilizes both 
carrier depletion and phase change material switching to support fast operation and wide tuning capability.

An ultracompact optical switch has been presented with serially coupled ring resonators that can be switched using optical phase change materials. The performances of this switch are compared with two different phase change materials and also compared with a directional coupler switch that uses the same switching scheme. Finally, the potential of large scale integration in a non-blocking architecture was investigated. 


\section{Acknowledgements}

I would first like to thank Professor Winnie Ye for her supervision and intellectual support throughout my Master's program. I am grateful for all the productive discussions on a wide range of photonic devices and motivating me to pursue multiple projects for my program. I am eternally indebted to Dr. Alan Scott, Dr. Hugh Podmore from Honeywell Aerospace to guide me on the Optical Phased Array (OPA) project. I also extend my gratitude to my fellow colleagues Dr. Dusan Gostimirovic, Tianyi Hao for involving me in crucial pedagogical discussions before deciding on my project. I would like to convey special thanks to Dr. Gostimirovic to collaborate with me on the optical logic project.

I am grateful to my family and friends who gave me constant support through thick and thin. 


\section{List of Symbols}

c Speed of light in vacuum

$n \quad$ Refractive index

$t$ Time

$e \quad$ Electronic charge

$m_{c e}^{*} \quad$ Effective mass of electron

$m_{c h}^{*} \quad$ Effective mass of hole

$\mu_{e} \quad$ Electron mobility

$\mu_{h} \quad$ Hole mobility

$N_{e} \quad$ Free electron concentration

$N_{e} \quad$ Free hole concentration

$n_{c o} \quad$ Refractive index of the core material

$n_{c l} \quad$ Refractive index of the cladding

$k$ Propagation constant

$A F \quad$ Array Factor

$I_{m} \quad$ Magnitude of element signals

$R_{\text {disk }} \quad$ Radius of disk resonator

$n_{\text {eff }} \quad$ Effective Refractive index

IL Insertion Loss 


\section{List of Abbreviations}

DNN Deep Neural Network. 38

EO Electro Optical. 8

FCD Free Carrier Dispersion. 5, 36

FPGA Field Programmable Gate Array. 1

FWM Four-wave mixing. 5

GSST $\mathrm{Ge}_{2} \mathrm{Sb}_{4} \mathrm{Se}_{2} \mathrm{Te}_{5} .8$

GST $\mathrm{Ge}_{2} \mathrm{Sb}_{2} \mathrm{Te}_{5} .8$

IL Insertion Loss. 58

LSI Large Scale Integration. 36

MZI Mach-Zehnder interferometer. 2

O-PCM Optical Phase Change Material. 8

OPA Optical Phased Array. 3

PIC Photonic Integrated Circuit. 1

PNN Photonic Neural Network. 36 
PSLL Peak Side Lobe Level. 21

SOI Silicon on Insulator. 1

TO Thermo Optic. 8

WDM Wavelength-Division Multiplexing. 4

XPM Cross-phase Modulation. 5 


\section{Table of Contents}

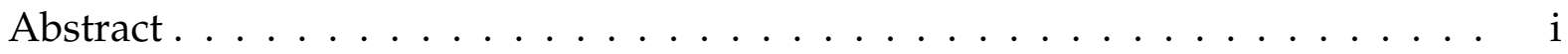

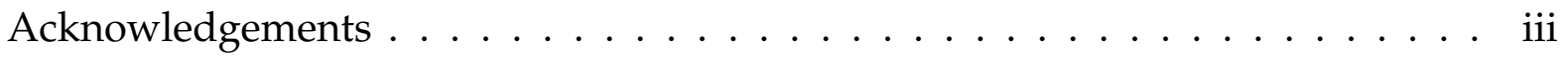

List of Symbols $\quad$ iv

List of Figures $\ldots \ldots \ldots \ldots \ldots \ldots \ldots \ldots \ldots \ldots \ldots \ldots$

List of Tables . . . . . . . . . . . . . . . . . . . . xvii

1 Introduction 1

1.1 Motivation . . . . . . . . . . . . . . . . . . 1

1.2 Optical Interconnection Networks in PICs . . . . . . . . . . . . . . . 4

1.3 Electronic Control of Photonic Components . . . . . . . . . . . . . . . . . 5

1.4 Phase Change Materials on Photonic Information Processing . . . . . . . . 8

1.5 Thesis Objectives and Orgranisation $\ldots \ldots \ldots \ldots$

2 Control and Optimization of Optical Phased Arrays (OPA) for Beam Steering 11

2.1 Phased Array Basics $\ldots \ldots \ldots \ldots$

2.2 Optical Phased Arrays . . . . . . . . . . . . . . . . . . . 15

2.3 Single Emitter Analysis . . . . . . . . . . . . . . . . . . . . . . 16

2.4 Far Field Simulation and Optimization of Antenna locations . . . . . . . . 20

2.5 Beam Steering Algorithm $\ldots \ldots \ldots$

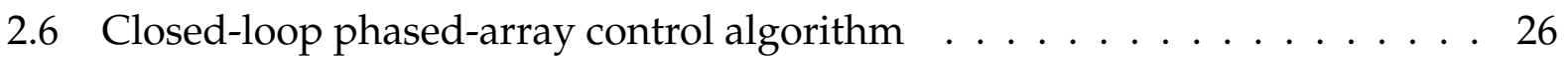

2.7 Experimental Setup and Results . . . . . . . . . . . . . . . . . . . 30

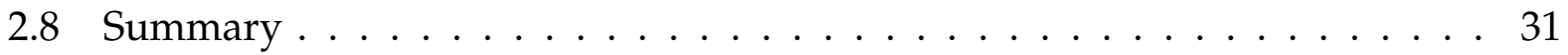


3 Reconfigurable Optical Logic Using Carrier Depletion and Optical Memory in Microdisk Resonators 36

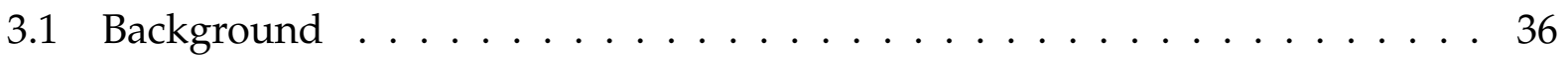

3.2 GSST cladded Disk Resonator . . . . . . . . . . . . . . . . . . . . . . . 39

3.3 Electrothermal Switching of O-PCM with ITO Heater . . . . . . . . . . . 42

3.4 Carrier Depletion in Silicon Microdisks . . . . . . . . . . . . . . . . 44

3.5 Switching and Logic Architecture . . . . . . . . . . . . . . . . . . . 45

3.6 Higher Order Logic . . . . . . . . . . . . . . . . . . . . . . . . . 48

3.6.1 N-x Logic Operation . . . . . . . . . . . . . . . . . . . . 48

$3.6 .2 \quad \mathrm{~N}$-bit Comparator . . . . . . . . . . . . . . . . . . . 48

3.6 .3 Optical Adder . . . . . . . . . . . . . . . . . . . . . . . . . 51

3.7 Summary . . . . . . . . . . . . . . . . . . . 51

4 Non-volatile Switching for Wavelength Selective Spatial Routing 53

4.1 Non-volatile Switching . . . . . . . . . . . . . . . . . . . . . 53

4.2 Wavelength-Selective Spatial Routing . . . . . . . . . . . . . . 54

4.3 Photonic Interconnection Network Architectures . . . . . . . . . . . . . . 55

$4.42 \times 2$ Photonic Switch with Coupled Ring Resonators . . . . . . . . . . . 56

4.5 Summary . . . . . . . . . . . . . . . . . . 61

5 Conclusion $\quad 63$

5.1 Summary of Thesis $\ldots \ldots \ldots \ldots \ldots$

5.2 Statement of Impact . . . . . . . . . . . . . . . . . . . . . . . 64

5.3 Future Works . . . . . . . . . . . . . . . . . . . . . 65

5.3.1 Improving Insertion Loss Performance $\ldots \ldots \ldots 5$

5.3.2 Full demonstration of our proposed control algorithm . . . . . . 66

5.3.3 Implementing Reconfigurable Switches on a Photonic Neural Network Architecture . . . . . . . . . . . . . . 66 


\section{List of Figures}

1.1 Typical silicon photonic optical transceiver with photonic components such as optical modulators (MOD), optical interface (I/O), power splitter/combiner (PS), wavelength filters (MUX/DEMUX), polarization control (PC) and photodetectors (PD). Reprinted from [13] . . . . . . . . . . . . . . . . . 2

1.2 a) A $2 \times 2$ optical gate. Here, $a_{1}(t)$ and $a_{2}(t)$ represent the input of the optical gate and $b_{1}(t)$ and $b_{2}(t)$ represent the output. b) The gate can be programmed at 'Bar', 'Cross' and 'Partial' (50-50 split) states, c),d) and e) The gate is programmed by controlling both the power coupling $\kappa$ and the phase delay $\Delta \phi$ through the use of phase shifters $p_{1}$ and $p_{2}$. Reprinted from $[4] \ldots \ldots \ldots \ldots \ldots \ldots \ldots$

1.3 Block diagram of a typical photonic interconnection link, reprinted from [3] 4

1.4 $4 \times 4 \times 4$ WDM crossconnect based on four cascaded $4 \times 4$ crossbar matrices that use active two-microring resonators, reprinted from [29] . . . . . . . . . 5

1.5 (a) Concentration of free electrons obtained through FEM simulations while $0 \mathrm{~V}$ and $-2 \mathrm{~V}$ biases are applied at the left and right electrodes respectively. (b) Wavelength shift due to biases applied at the electrodes obtained through FEM-FDTD simulations, reprinted from [17] $\ldots \ldots \ldots$ 
1.6 An application of phase change material: Tunable gradient metasurface. (a) Schematic of the tunable metasurface consisting of an array of GST nanorods with with different phase states and a Au mirror separated by a spacer layer. The reflection angle can be switched by modulating the phase state of the GST nanorods. (b) A three-level gradient phase modulation can be achieved by switching the GST nanorods. Reprinted from [14] . . . . . . 9

2.1 The geometry related to one radiator with excitation $V_{n} \ldots \ldots \ldots$

2.2 Ray diagram for linear receiving array, Reprinted $[20] \ldots \ldots$. . . . . . 14

2.3 Circular array of emitters, Reprinted [31] . . . . . . . . . . . . . . . . 15

2.4 Components of a typical OPA . . . . . . . . . . . . . . . . . 15

2.5 OPA with tunable laser source. The phase shifters and tunable laser both are used to steer the output in two directions. Schematic of the device showing only five channels for clarity. The red arrows indicate the direction of light flow. The beam is steerable along the $\theta$ direction using wavelength tuning and the phase shifters are used to steer in the $\psi$ direction. Reprinted $[21] \ldots \ldots \ldots \ldots$

2.6 A. Chip micrograph of a $4 \times 4$ OPA with fixed wavelength. Light after getting coupled through a grating coupler is guided to a network of Ysplitters. The network is then split into $16 \mathrm{p}-\mathrm{i}-\mathrm{n}$ phase modulators and finally fed into 16 grating couplers. The $4 \times 4$ OPA is designed with a rectangular arrangement. B. The projected far field pattern generating a sad face. Reprinted [1] . . . . . . . . . . . . . . . . . . . . . . 18

$2.7 \quad \mathrm{X}$ coordinates $(\mathrm{m})$ vs Intensity $\left(W / m^{2}\right) \ldots \ldots \ldots \ldots$ 
2.8 Far field pattern of a $3 \times 9 \mu m^{2}$ (a) rectangular aperture and (b) grating coupler of same dimensions. Here $U=\sin (\theta) \cos (\phi)$ and $V=\sin (\theta) \sin (\phi)$ where $\theta$ and $\phi$ are polar and azimuthal angles respectively. In a spherical coordinate system, the azimuthal angle is defined as the orthogonal projection of a point on a reference plane that passes through the origin and is orthogonal to the zenith, measured from a fixed reference direction on that plane. . . . . . . . . . . . . . . . . . . . . . . . . . 19

2.9 Schematic of emitter region of dual ring OPA . . . . . . . . . . . 20

2.10 Far Field Intensity profile with 100 emitters . . . . . . . . . . . . . . 21

2.11 Scan of power through 0 degree elevation angle with varying azimuthal angle for single ring OPAs of radii $125 \mu \mathrm{m}$ and $75 \mu \mathrm{m} \ldots \ldots$. . . . . . 21

2.12 Scan of power through 0 degree elevation angle vs azimuthal angle for dual ring OPAs with fixed inner ring radius at $43.5 \mu \mathrm{m}$ and outer ring radii at 66

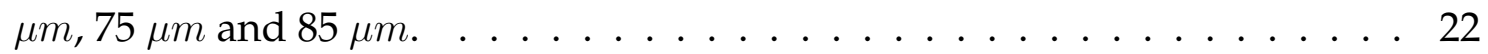

2.13 Peak Sidelobe level (PSLL in $\mathrm{dB}$ ) vs inner ring radius with outer ring radius fixed at $200 \mu m \ldots \ldots \ldots \ldots \ldots$

2.14 Optimizing the element position of four ring OPA $\ldots \ldots$. . . . . . . 23

2.15 Flowchart of the optimization process, reprinted from [1] . . . . . . . . . 24

2.16 Intensity profile of a steered beam at $4^{\circ} \ldots \ldots \ldots \ldots$

2.17 Scan of power in $\mathrm{dB}$ vs azimuthal angle for $0^{\circ}$ elevation cut with a) $0^{\circ}$ steer-

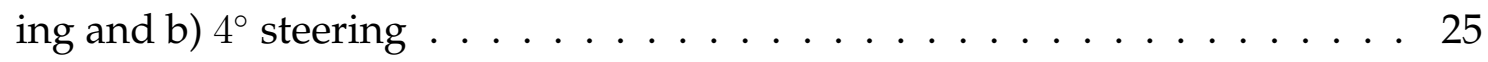

2.18 Steering angle vs PSLL $($ in $\mathrm{dB}) \ldots \ldots \ldots \ldots \ldots \ldots$

2.19 The proposed control algorithm . . . . . . . . . . . . . . . 26

2.20 The phase of all the emitters are modulated with a sinusoid signal. One of the emitters have a certain phase error. This phase error can be traced in

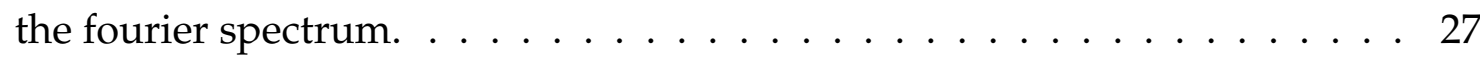


2.21 a) Time domain and b) Frequency domain signal that acts as an input into the 100 element OPA. All the emitters are modulated with frequencies between $92 \mathrm{~Hz}$ to $191 \mathrm{~Hz}$ with $1 \mathrm{~Hz}$ seperation. . . . . . . . . . . . . . . 27

2.22 Frequency domain output (in $\mathrm{Hz}$ ) of intensity pattern with all of the emitters modulated with a sinusoid signal (No bias applied at any of the emitters). We can see that there are no peaks between $92 \mathrm{~Hz}$ and $191 \mathrm{~Hz}$. . . . . . 28

2.23 Frequency domain output (in $\mathrm{Hz}$ ) of intensity pattern with all of the emitters modulated with a sinusoid signal with a) The emitter with $191 \mathrm{~Hz}$ has extra $45^{\circ}$ phase bias b) Four emitters are at $30^{\circ}$ phase bias. The four emitters are modulated at 168,172, 176 and $180 \mathrm{~Hz}$. We see the peaks in Fourier domain with a reduced intensity in comparison to $45^{\circ}$ phase bias. . . . . . 29

2.24 Micrograph of the 8 element OPA provided by Honeywell . . . . . . . . 31

2.25 Schematic of Ground Station Equipment (GSE) Setup . . . . . . . . . . . 32

2.26 Circuit diagram of voltage controlled current source to drive the phase shifters . . . . . . . . . . . . . . . . . . . 32

2.27 a) Photo of our experimental setup for OPA chip characterization, b) Output signal seen from a lumenera camera . . . . . . . . . . . . . 33

2.28 Experimental results of 8 element OPA where four emitters are modulated with frequencies $4 \mathrm{~Hz}, 5 \mathrm{~Hz}, 6 \mathrm{~Hz}$ and $7 \mathrm{~Hz}$. The emitter with $7 \mathrm{~Hz}$ has a) $2 \mathrm{~V}$ and b) $0.5 \mathrm{~V}$ constant bias. The upper graphs are plotting the summation of the pixel values of the OPA region vs number of frames. The lower graphs are showing the values in the frequency spectrum. . . . . . . . . . 34

2.29 Experimental results of 8 element OPA where three elements are modulated. The element under bias can be detected from the intensity spectrum. Same as figure 2.28 The upper graphs are plotting the summation of the pixel values of the OPA region vs number of frames. The lower graphs are showing the values in the frequency spectrum. . . . . . . . . . . 35 
3.1 a) Optical transmission of a phase change material in amorphous and crystalline states. The amorphous and crystalline states represent write and erase states respectively. As seen from the level scheme, crystalline state is more absorptive than amorphous. The insets show the atomic ordering of the states. Reprinted from [26] b) Cohesive energy plot of cubic and orthorhombic phases of Ge-Sb-Se-Te (GSST)- a phase change material with varying concentration of Se. Reprinted from [36] . . . . . . . . . . . . 38

3.2 (a)Schematic of GSST on Silicon Microdisk with ITO heater on top. (b) Cross-section of the right half of the disk. (c) Evolution of a quasi-TE mode traversing along a Si waveguide with GSST on top. (d) Demonstration of redshift of resonance wavelength with changing the GSST from amorphous to crystalline. The orange and blue plot indicates response in amorphous and crystalline states respectively. Lumerical's FDTD simulation were performed on a Silicon microdisk of $5 \mu \mathrm{m}$ radius and $0.22 \mu \mathrm{m}$ height. Other parameters are: $\mathrm{L}_{\mathrm{GSST}}=1.5 \mu \mathrm{m}, \mathrm{W}_{\mathrm{GSST}}=0.4 \mu \mathrm{m}, \mathrm{H}_{\mathrm{GSST}}=0.025 \mu \mathrm{m}$, height of ITO layer is $0.01 \mu \mathrm{m}$. The amount of redshift we see is around $10 \mathrm{~nm}$. . . 40

3.3 (a) Transmission spectra is redshifted as we gradually increase $\mathrm{L}_{\mathrm{cGSST}}$. As the length of the GSST is material is increased, it absorbs more light and shifts the value of effective refractive index causing the spectrum to be redshifted. b) The shift of the resonance peak with gradual increase in the $\mathrm{L}_{\mathrm{cGSST}}$ or $\mathrm{L}_{\mathrm{aGSST}}$. A plot like this helps us identify the length of GSST material required to achieve a certain shift in resonance. . . . . . . . . . . 41 
3.4 Electro-thermal switching from amorphous to crystalline through Joule heating and vice versa. a) Heat map produced by Joule heating. b) Temperature average in the GSST layer as a function of time for rectangular pulse heater. For amorphization, the temperature is kept above $900 \mathrm{~K}$ (amorphization temperature) by applying a single rectangular pulse of 24 V. c) For crystallization, the average temperature is kept above $523 \mathrm{~K}$ (crystallization temperature) but below $900 \mathrm{~K}$ for more than $20 \mu \mathrm{s}$. . . . . . . . . 43

3.5 (a) Schematic of the double-shift vertical PN junction. The graded region indicate the metal contacts to switch the microdisk. (b) Cross section of the microdisk buried in oxide layer with $\mathrm{p}, \mathrm{n}, \mathrm{p}+$ and $\mathrm{n}+$ regions defined. . . . 45

3.6 a) Carrier profile of free electrons of the vertical junction simulated through FEM simulation when $3.4 \mathrm{~V}$ of reverse bias is applied on the right of the microdisk. The right side of the disk shows a $45 \mathrm{~nm}$ of larger depletion width. b) Transmission response at the drop port of the microdisk with applied biases of $0 \mathrm{~V}$ and $3.4 \mathrm{~V} \ldots \ldots \ldots \ldots \ldots$

3.7 a) Schematic of the logic cell with $2 x$ logic operations. The PCM strip has a length of $400 \mathrm{~nm}$, a width of $200 \mathrm{~nm}$ and $25 \mathrm{~nm}$ of height. b) Transmission response under different states of PCM and PN-junction. Two logic operations can be carried out at wavelength $1.571 \mu \mathrm{m} \ldots \ldots \ldots \ldots$

3.8 (a) Schematic of a logic cell with $3 x$ operations in a single disk and at a fixed wavelength. This cell can be scaled up to nx operations. (b) Transmission response of a logic cell of similar functionality. The blue, red and green curves represent amorphous state with $0 \mathrm{~V}$ bias, mixed state with $1.7 \mathrm{~V}$ bias and crystalline state with $3.4 \mathrm{~V}$ bias respectively. The black and purple curves represent amorphous states with $1.7 \mathrm{~V}$ and $3.4 \mathrm{~V}$ applied Bias. We can see that by changing the state of the PCM we can shift the resonance peak and align them with the initial state. This can be utilized for our $3 x$ operation logic cell. . . . . . . . . . . . . . . . 
3.9 Schematic of a 4-bit comparator. The through port determines if two 4-bit numbers (A and $B$ ) are equal. The drop ports determine if $A$ is greater than B or not. . . . . . . . . . . . . . . . . . . . . . . . . . . . . 50

3.10 Schematic of a 2-bit optical adder. For $\mathrm{N}$ bit operations this cell would require $3 \mathrm{~N}-3$ disks. . . . . . . . . . . . . . . . . . . . . . . 52

4.1 Routing techniques available on photonic interconnection networks, reprinted [8]

4.2 (a) A $4 \times 4$ TorusNX topology reprinted from [2]. The $G$ labels represent the gateways and $X$ represent $4 \times 4$ non-blocking switches. (b) Layout of the gateway. (c) Layout of the $4 \times 4$ non-blocking switch. . . . . . . . . . . . 56

4.3 (a) Schematic of an $N \times N \times M$ switch with L Micro Ring Resonators per crosspoint (b) Unit cell of the switch (c) Layout of an $8 \times 4$ switch (d) Die Shot of the switch with I/O marked. Reprinted from [22] . . . . . . . . . . 57

4.4 Optical constants of (a) amorphous and (b) crystalline phases of GST (solid lines) and GSST (dashed lines) from the visible range to near-infrared. The black lines represent the refractive index $n$, and the red curves are the extinction coefficient k. Reprinted [35] . . . . . . . . . . . . . . . . . 58

4.5 Proposed 2x2 switch in [35]: (a) cross section and (b) perspective view of the switch. Here $w_{c}=512 \mathrm{~nm}, w_{s}=730 \mathrm{~nm}, w_{p}=400 \mathrm{~nm}, w_{g}=562 \mathrm{~nm}, \mathrm{~h}=$ $450 \mathrm{~nm}$, and $h_{p}=60 \mathrm{~nm}$; (c) and (d) FDTD simulated field intensity distribution in the device at the (c) amorphous (cross) and (d) crystalline (bar)

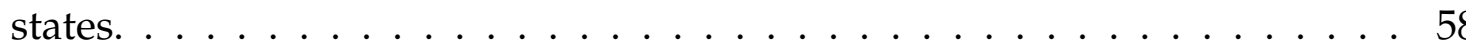

4.6 Proposed $2 \times 2$ ring resonator-based switch. Red and yellow indicate amorphous and crystalline states of the GST, respectively. (a) and (b): different states of the switch; (c) wavelength-selective crossbar switch. . . . . . . . . . 59

4.7 Modal intensity profiles of a Si-waveguide cladded with a GST strip in the (a) amorphous and (b) crystalline states. (c) FDTD simulated field intensity distribution in the amorphous state. 
4.8 Transmission response with (a) GST and (b) GSST overlays for the device proposed in $4.4 \ldots \ldots \ldots \ldots \ldots$

4.9 Schematic of a possible $2^{m} \times 2^{m}(\mathrm{~m}=2)$ Benes network switch. The blue path indicates a possible all-cross state. . . . . . . . . . . . . . . 60

4.10 Insertion loss of our proposed switch network at $1554 \mathrm{~nm} . \ldots 61$

5.1 Wrapped waveguide dual microdisk switches $\ldots \ldots \ldots 6$

5.2 An electro-optic neuron concept taken from [15]. They operate taking input from a WDM bus relayed through a set of ring resonators or from a parallel bus containing optical interferometers then converted to an electrical signal from optical signal with a photodiode. The signal then amplified by a TIA modulates a CW laser that produces a non-linear transfer function at the

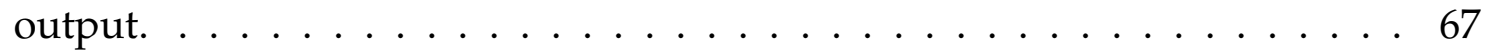

5.3 Gaussian fit of simulated data points for the device presented in Fig. 4.6. . . 67 


\section{List of Tables}

3.1 Parameters used for thermal simulations in COMOSOL Multiphysics . . . 43

3.2 Blueprint for $2 x \operatorname{logic}$ operation $\ldots \ldots \ldots \ldots$

3.3 Blueprint for $3 x \operatorname{logic}$ operation $\ldots \ldots \ldots \ldots$

4.1 Performance Comparison of $2 \times 2$ switch with double ring resonators _ . . 62 


\section{Chapter 1}

\section{Introduction}

\subsection{Motivation}

Photonic integrated circuits (PICs) have recently been pivotal for applications in communication, sensing and computing. Inspired from integrated circuits (ICs), PICs use photons instead of electrons and provides significant benefits in terms of size, weight, power and cost. On-chip optical waveguides, electro-optic modulators, optical couplers, lasers and photodetectors are mostly used to manipulate light in a PIC. PICs are mostly used in fiber-optic communications, over a broad range of transmission distances, but they are also useful in various applications such as quantum information processing, photonic logic, optical FPGA (Field Programmable Gate Array), photonic neural networks and biological or spectroscopic sensing. [4]

Silicon photonics is an excellent candidate for PIC owing to their compatibility with CMOS electronics fabrication processes. Silicon is transparent to wavelengths commonly used for optical transport (around $1.3-1.6 \mu \mathrm{m}$ ) and thus Silicon photonics is the ideal candidate for guiding light in a PIC. Silicon-on-insulator (SOI) is the typical platform to guide and control light in PICs. Figure 1.1 shows a Silicon photonic transceiver containing

optical passives such as couplers, routers, interferometers and actives such photodetectors and modulators. Most optical actives are made from III-V-based compounds such as 


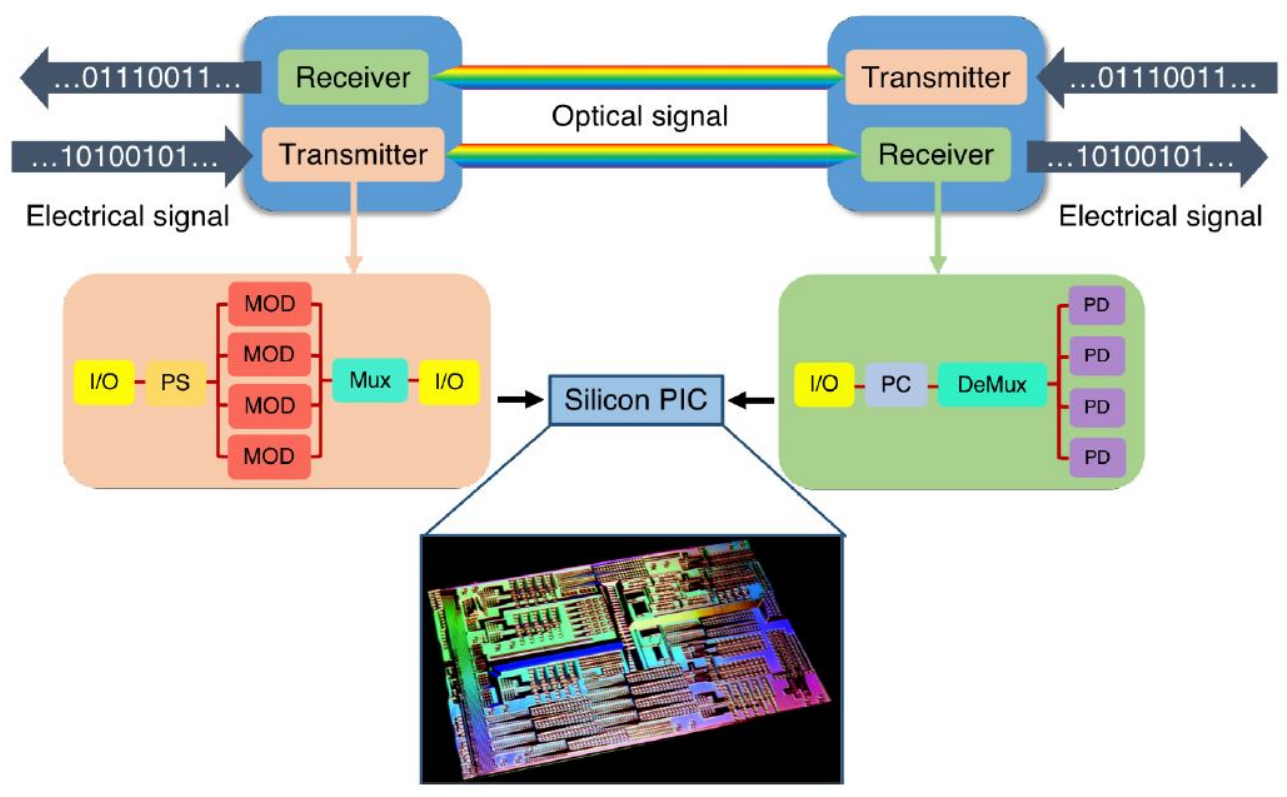

Figure 1.1: Typical silicon photonic optical transceiver with photonic components such as optical modulators (MOD), optical interface (I/O), power splitter/combiner (PS), wavelength filters (MUX/DEMUX), polarization control (PC) and photodetectors (PD). Reprinted from [13]

gallium arsendie (GaAs), indium photosphide (InP) or the electro-optical crystal lithium niobate $\left(\mathrm{LiNbO}_{3}\right)$. Recent advances has allowed us to integrate these active devices on a SOI platform through hybrid integration. Thus, SOI platforms have the potential to integrate photonic devices with existing electronics platforms as well as move to an all-optical platform. [13]

In a programmable PIC, the flow of light is controlled by placing various interferometric elements such as Mach-Zehnder Interferometers (MZIs), wavelength filters such as ring resonators, Bragg reflectors, and tuning the effective refractive index of the guided mode by applying active electrical or optical switching. Figure 1.2 shows an example of a $2 \times 2$ optical gate where the two optical outputs can be controlled by tuning the phase shifters $p_{1}$ and $p_{2}$. Suffice it to say, phase shifters are the key building block of programmable PICs and they can be realized using electrically controlled thermo-optic 


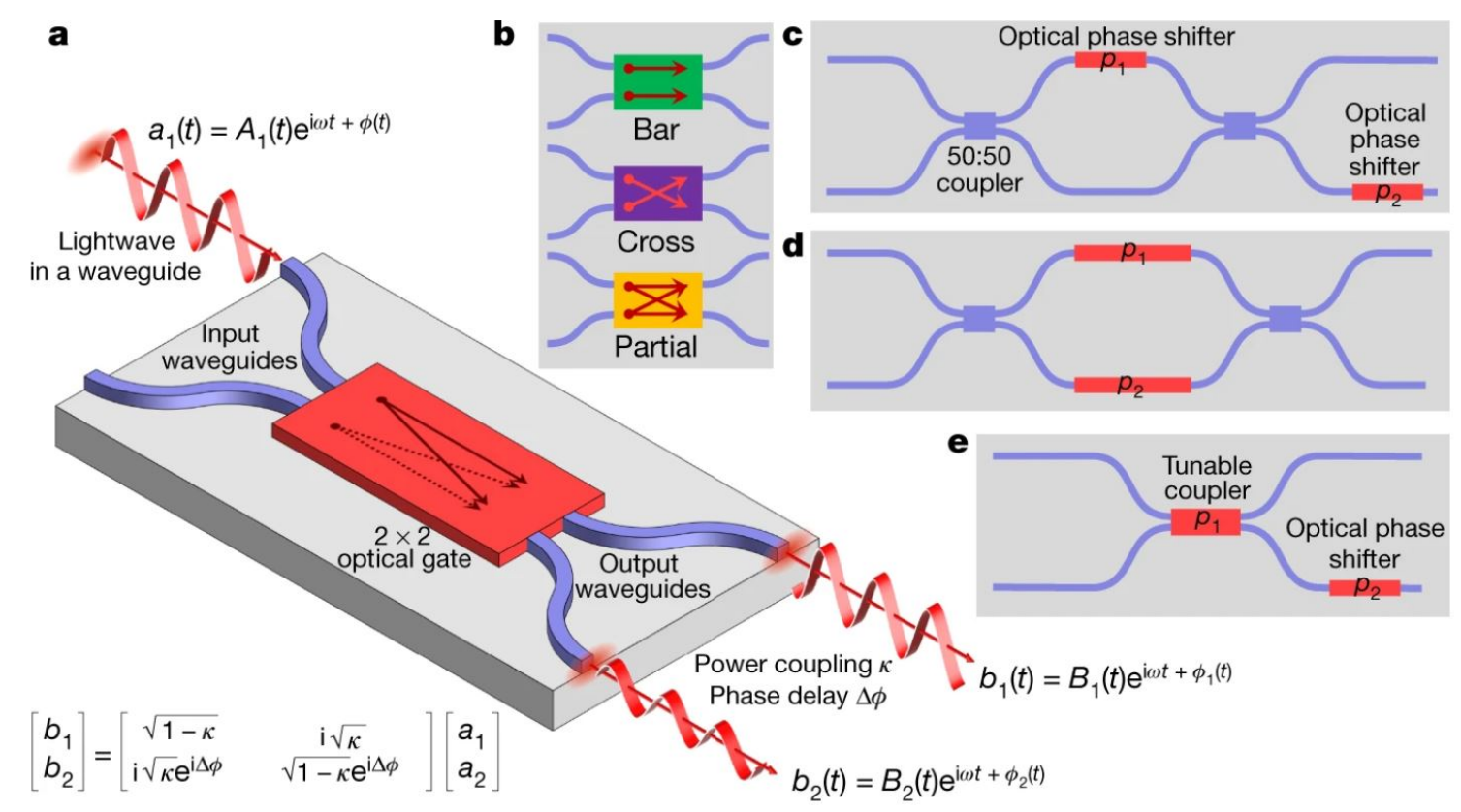

Figure 1.2: a) A $2 \times 2$ optical gate. Here, $a_{1}(t)$ and $a_{2}(t)$ represent the input of the optical gate and $b_{1}(t)$ and $b_{2}(t)$ represent the output. b) The gate can be programmed at 'Bar', 'Cross' and 'Partial' (50-50 split) states, c),d) and e) The gate is programmed by controlling both the power coupling $\kappa$ and the phase delay $\Delta \phi$ through the use of phase shifters $p_{1}$ and $p_{2}$. Reprinted from [4]

heaters, free carrier injection in semiconductor, materials with Pockels effect, phase change properties and optical non-linearities. [4]

In this thesis work, we propose design and control methodologies of programmable PICs with different applications. Novel switching architectures are proposed using phase change materials and carrier injection in Silicon that can be implemented in optical routing and logic applications. We also discuss, optical beamforming techniques with Optical Phased Arrays (OPA) and propose a novel control algorithm to perform beam steering and phase noise suppression. The following chapters provide a background on carrierinjection, phase-change materials and conclude with a brief summary of research objectives and contributions. 


\subsection{Optical Interconnection Networks in PICs}

Optical interconnection networks provide a significant advantage over electronic ICs in terms of footprint and bandwidth. Use of wavelength-division multiplexing (WDM) in photonic integrated circuits allows huge data streams to be sent in a single waveguide. As shown in figure 1.3 a complete interconnection network consists of three main sections generation, routing and reception. On-chip coherent light generation can be obtained by monolithically integrated hybrid III-V/silicon lasers. The key components for optical routing are couplers, waveguides, modulators and wavelength filters. Optical taps and photodetectors can be used to receive the encoded information in light [3].

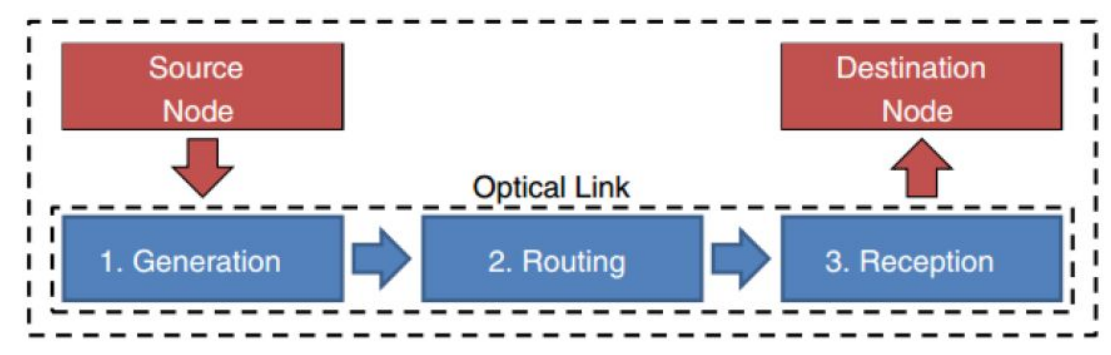

Figure 1.3: Block diagram of a typical photonic interconnection link, reprinted from [3]

Most of the interconnection networks use resonator structures for routing since they provide compact footprint and efficient energy consumption. These networks can generally be classified as either wavelength routing or spatial routing for guiding the coherent light through the chip. Wavelength routing involves the use of wavelength filters like ring resonators or Bragg reflectors and transmit to any destination by selecting the appropriate wavelength channel. Spatial routing on the other hand involves guiding an entire spectrum of channels in WDM link through the use of various switching mechanisms. Figure 1.4 shows a nonblocking $4 \times 4 \times 4$ crossconnect switch that contains $4 \times 4$ crossbar matrices each containing 4 distinct wavelengths. This is an example of wavelength-selective spatial routing where spatial routing is enhanced by dividing the wavelength channels into several WDM partitions. Here the microring resonators meet two purposes. First, they work as passive networks that help to route the signals toward certain destinations 
and second, as active networks which selectively filter certain wavelengths by tuning the resonators.

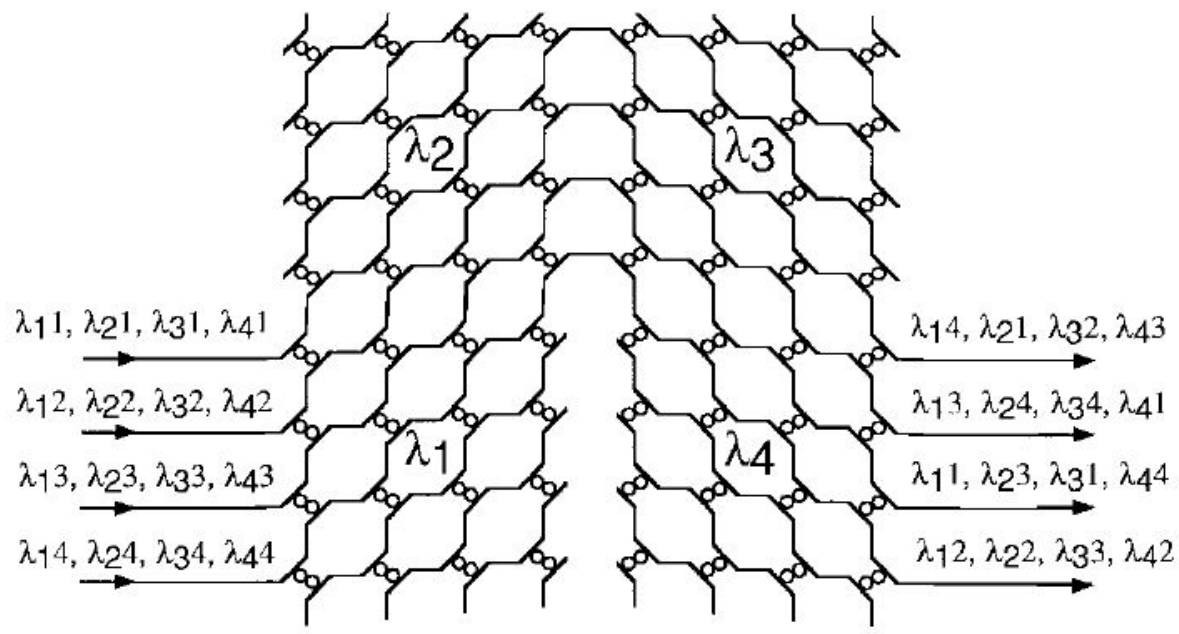

Figure 1.4: $4 \times 4 \times 4$ WDM crossconnect based on four cascaded $4 \times 4$ crossbar matrices that use active two-microring resonators, reprinted from [29]

\subsection{Electronic Control of Photonic Components}

One of the key requirements of programming PICs is tune the properties of the guided light. This can be done by applying an optical or electrical signal that induces a change in the refractive index of the material that guides the optical signal. High speed modulation is also achieved through optical nonlinearities such as cross-phase modulation (XPM) and four-wave mixing (FWM) in SOI waveguides. Nonetheless, it is widely accepted that the most efficient mechanism for optical switching is achieved through free-carrier dispersion (FCD) by injection or depletion. FCD effects change both the real and imaginary indices of the material. Derived from the Drude-Lorenz equation, the change in refractive index can be obtained from the following equation [25]:

$$
\Delta n=\frac{-e^{2} \lambda_{0}^{2}}{8 \pi^{2} c^{2} \epsilon_{0} n}\left(\frac{N_{e}}{m_{c e}^{*}}+\frac{N_{h}}{m_{c h}^{*}}\right)
$$


here, $c$ represents the speed of light in vacuum, $e$ the electronic charge, $\mu_{e}$ electron mobility, $\mu_{h}$ hole mobility, $m_{c e}^{*}$ effective mass of electrons, $m_{c h}^{*}$ effective mass of holes, $N_{e}$ the free electron concentration, $N_{h}$ the free hole concentration and $\lambda_{0}$ the free space wavelength. Figure 1.5 shows the simulations results of microdisk modulators when they are tuned with FCD effects. It is seen that it is possible to achieve $45 \mathrm{pm} / \mathrm{V}$ of resonance peak shift with energy consumption of $0.9 \mathrm{fJ} /$ bit.

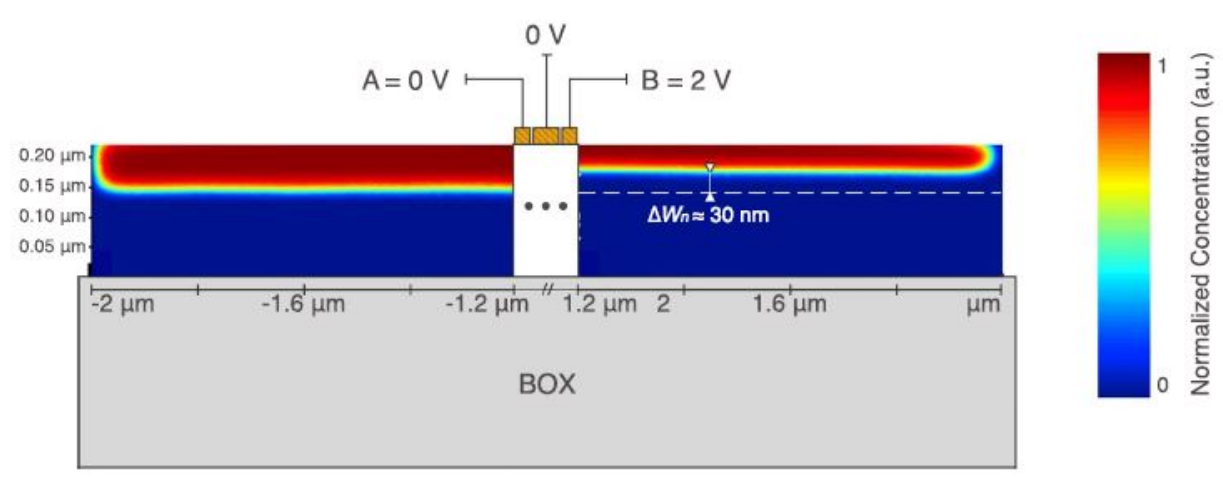

(a)

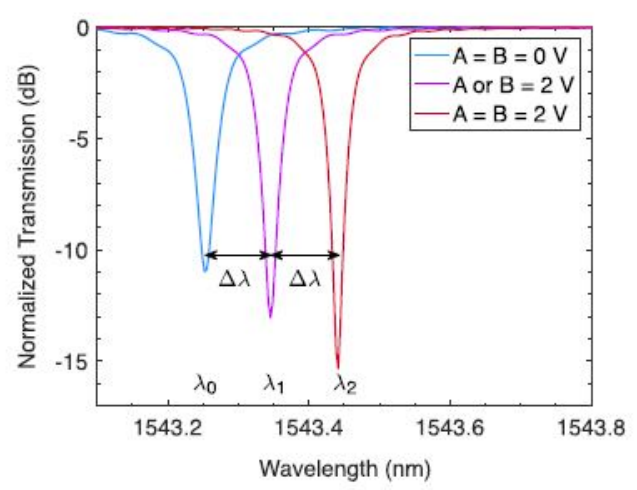

(b)

Figure 1.5: (a) Concentration of free electrons obtained through FEM simulations while 0 $\mathrm{V}$ and $-2 \mathrm{~V}$ biases are applied at the left and right electrodes respectively. (b) Wavelength shift due to biases applied at the electrodes obtained through FEM-FDTD simulations, reprinted from [17]

For applications where wider tuning is required such as in Optical Phased Arrays (OPA), thermal tuning is a good option since they apply a wide bandwidth of operation. A change of effective refractive index can be achieved by locally heating the waveguide 
at its core and cladding. Let us consider $n_{c o}$ and $n_{c l}$ be the refractive index of the core and cladding respectively. Thus, the at certain temperature $\mathrm{T}$, the refractive index of core of cladding:

$$
\begin{aligned}
& n_{c o}(T)=n_{c o}\left(T_{0}\right)+\frac{d n_{c o}}{d T} d T \\
& n_{c l}(T)=n_{c l}\left(T_{0}\right)+\frac{d n_{c l}}{d T} d T
\end{aligned}
$$

where $d T=T-T_{0}$ with $T_{0}$ being the room temperature and $\frac{d n_{c o} / c l}{d T}$ is the thermo-optic coefficient of the core/cladding material. The thermo-optic coefficient of Silicon is $1.86 \times$ $10^{-4} / \mathrm{K}$ that means if the waveguide material can be raised by $6 \operatorname{deg} C$, the refractive index changes by $1.1 \times 10^{-3}$.

The temperature of the waveguide material can be changed by applying heat through local resistive heating of a metal layer, called heater or thermo-optic phase shifter. The temperature variation $d T$ associated with the current flow can be determined as:

$$
d T(t)=\frac{P . t}{m \cdot C}
$$

where $\mathrm{m}$ is the mass of the heater, $\mathrm{t}$ is the time, $\mathrm{P}$ is the electrical power generated and $\mathrm{C}$ is heat capacity of the metal. The heat generated at the top cladding thus transmits to the core and the temperature variation induces a phase delay to the propagating light. The amount of phase change as a function of time can be expressed as:

$$
\Delta \phi(t)=\frac{2 \pi D}{\lambda_{0}} \frac{d n}{d T} d T(t)
$$

where $\mathrm{D}$ is the length of the device, $\lambda_{0}$ is the free-space wavelength, $d T$ is the change in temperature and $\frac{d n}{d T}$ is the thermo-optic coefficient. 


\subsection{Phase Change Materials on Photonic Information Pro- cessing}

Silicon photonic devices can be actively tuned by widely established thermo-optic (TO) or Electro-Optic (EO) effects which has a refractive index tuning range in the order of 0.01 and 0.0001 respectively. Thus, a large phase shift usually requires long waveguides of around hundreds of micrometers increasing the footprint and complexity of the device. Employing a resonator structure can circumvent this problem although it will at the cost of device bandwidth. Furthermore, these switching mechanisms are volatile and requires constant power supply. Devices based on optical phase change (O-PCM) materials have been studied because of their large index change properties and reconfigurability. The phase change introduced by these materials can be nonvolatile and can be transitioned from amorphous to crystalline state. Recent research has also shown that, some (O-PCM) materials like $\mathrm{Ge}_{2} \mathrm{Sb}_{2} \mathrm{Te}_{5}$ (GST) can be tuned quasi-continuously and the intermediate states between amorphous and crystalline state can be achieved at room temperature.

Chalcogenide PCMs can be reversibly switched by low-energy optical or electrical pulses with sub-ns timescale with long endurance up to $10^{15}$ cycles. Also, PCMs are higly scalable and can be simply deposited onto any substrate without lattice mismatch. Thus, PCMs are being used in low-energy, compact footprint and broadband programmable PICs for memories, switches and computing. Despite having high switching bandwidth, O-PCMs are usually held back by high optical absorption [26]. Recently, new O-PCMs have been derived with simple engineering of traditional GST materials. Ge-Sb-Se-Te or GSST have been derived from conventional GST by partially substituting Te with Se.

\subsection{Thesis Objectives and Orgranisation}

This thesis is focused on design, simulation and control of photonic integrated circuits for application in optical phased arrays (OPA), reconfigurable computing and optical rout- 
(a)

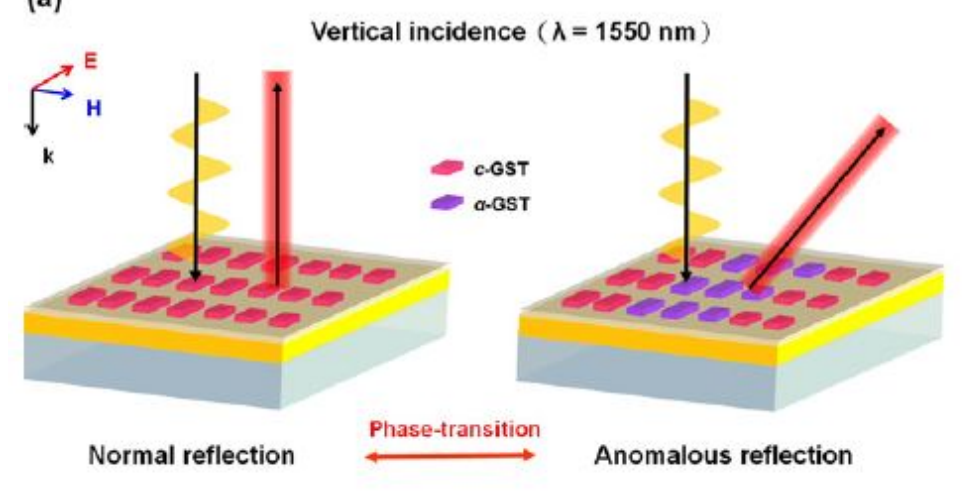

(b)

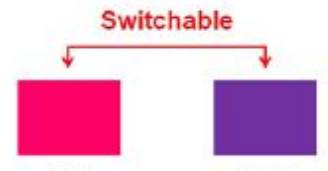

$\left(0^{\circ}\right)$

(120)

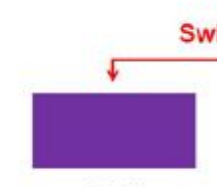

$\left(240^{\circ}\right)$
Switchable

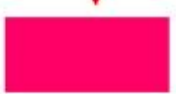

$\left(0^{\circ}\right)$

Equivalent

Figure 1.6: An application of phase change material: Tunable gradient metasurface. (a) Schematic of the tunable metasurface consisting of an array of GST nanorods with with different phase states and a Au mirror separated by a spacer layer. The reflection angle can be switched by modulating the phase state of the GST nanorods. (b) A three-level gradient phase modulation can be achieved by switching the GST nanorods. Reprinted from [14]

ing. The main improvements come from the proposition and demonstration of a novel algorithm that can aid the control of an optical phased array. In an effort to design seamlessly low power, high speed and high bandwidth optical devices for computing and routing, phase change materials have been introduced in the design. Their ability to change their physical state reversibly allows door for reconfigurable optical logic and non-blocking architectures.

In chapter 2 of this thesis we developed a script to optimize the arrangement of optical nanoantennas the fundamental building block of OPAs. Sections 2.1 through 2.3 presents the background required to develop the script. Section 2.4 presents the optimization method through the analysis of far-field intensity pattern simulated for each 
optical nanoantenna. Then in section 2.5 , a gradient descent based beam steering algorithm were described. In section 2.6 a closed-loop phased-array control algorithm were presented based on the frequency analysis of phase-modulated emitters and made comparisons with the more popular gradient descent based approach. In section 2.7, we verified some of our simulation results through experimental data obtained from an 8 element circular OPA.

In chapter 3, we present a reconfigurable optical logic architecture using carrier depletion effects and phase change materials. Sections 3.1 through 3.3 described the switching dynamics of phase change material cladded disk resonators. Then we delve into carrier depletion mechanism of switching in section 3.4. We then propose optical logic cells that can be designed keeping both mechanisms on the same disk in section 3.5. Finally, we propose some higher order logic architectures that can be suitable with our proposed logic cells.

In chapter 4 , we also proposed a $2 \times 2$ non-volatile switch based on serially coupled ring resonators with compact footprint and improved insertion loss performance. Our design is more compact compared to traditional directional coupler based switches.

In Chapter 5, a conclusion is made based on the designs and results presented in this thesis. In section 5.1 an overall summary is given on the work done for this thesis. In 5.2, several ideas were presented for future work. Finally, we provided an impact statement in section 5.3 . 


\section{Chapter 2}

\section{Control and Optimization of Optical}

\section{Phased Arrays (OPA) for Beam Steering}

\subsection{Phased Array Basics}

Phased arrays, well known in radio frequency have gained recent interest in optical frequencies owing to it's large-scale integration capabilities. While they have a wide range of applications, they can be viable for space-based communication since the generated beam can be electronically steered without any need for mechanical pointing strategies. Thus, the primary motivation behind this project is to develop a wafer scale integrated circuit that has the capability to replace fast steering mirrors in optical inter-satellite links reducing cost and footprint.

Mathematically a phased array is basically an N-slit diffraction example. We can consider each of these slits as a pixel in a phased array and we have the freedom to assign optical phase to each of these pixels [31]. The far-field radiation pattern of this system is the multiplication of the far field of an individual nanoantenna and that of the array factor.

Before discussing circular arrays, we begin the fundamentals with linear arrays since several aspects of circular arrays are built on the theory behind linear arrays. The basic 
linear array has a number of discrete radiating elements, equally oriented and equally spaced along a straight line. Each element is viewed as an electric or magnetic current source, which gives rise to a radiated field, the solution to Maxwell's equations. We assume that the normalized radiation function for one such element, n, is $E l_{n}(r)$ where $r$ is the direction and distance from a reference point in the element to the field point. The reference point has the global coordinates $\left(R_{n}\right)$, and the complex excitation of the element is $V_{n}$. Thus, the radiated field from this element, including polarization, is given by $V_{n}\left(E l_{n}\right)(r)$. Summing over all elements, we obtain the radiated field at the field point $\mathrm{P}$ given by the coordinates (Fig 2.1)

$$
E(r)=\sum_{n} V_{n} E l_{n}\left(r-R_{n}\right) e^{-j k\left|r-R_{n}\right|}
$$

Here, $\mathrm{k}$ is the propagation constant.

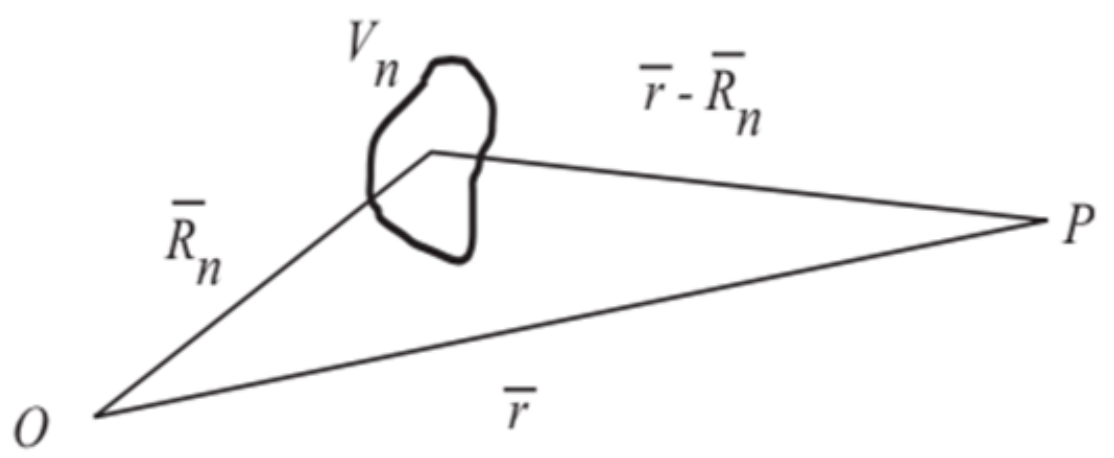

Figure 2.1: The geometry related to one radiator with excitation $V_{n}$

In the far field ( $\mathrm{r}$ large), the dependence of the amplitude on the distance will be approximately the same for all radiators. Since the elements were assumed to be identical and identically oriented, we can bring the element radiating function, except the relative phases, outside the summation in Eq. 2.1 and we get the far-field equation: 


$$
E(r)=E l(r) \sum_{n} V_{n} e^{-j k\left|r-R_{n}\right|}
$$

Discarding the common phase and amplitude dependence we write the radiation function in the following form:

$$
E(\theta, \phi)=E l(\theta, \phi) \sum_{n} V_{n} e^{-j k \cdot R_{n}}=E l(\theta, \phi) A F(\theta, \phi)
$$

where $(\theta, \phi)$ are the angular coordinates and AF is the array factor. In phased arrays most focus is placed on the array factor since the important figures of merit such as beamwidth, sidelob, radiation pattern are mostly dependent on the array factor.

Now, we consider that each element in a phased array is excited with a signal at an amplitude of 1, but since the transmission paths between the elements are not equal, the phase shift of each element will be different. Thus, array factor:

$$
A F=e^{j \epsilon_{0}}+e^{j \epsilon_{1}}+e^{j \epsilon_{2}}+\ldots+e^{j \epsilon_{N-1}}
$$

where, $\epsilon_{N}$ are the phases of an incoming plane wave at the element locations $\mathrm{m}=0,1, \ldots$, referenced to some point such as the origin.

Now, a case is considered where all of the elements of the array are separated by the same distance $d$. From figure 2.1, we can see that the phase of element $\mathrm{m}+1$ leads that phase of element $\mathrm{m}$ by $k \cdot d \cdot \cos (\theta)$, since the path length to element $\mathrm{m}+1$ is $d \cdot \cos (\theta)$ meters longer than that to m. If we arbitrarily set the reference point to element 0 , so that $\epsilon_{0}=0$, so we can write

$$
A F=1+e^{j k d \cos (\theta)}+e^{j k 2 d \cos (\theta)}+\ldots+e^{j k(N-1) \operatorname{dcos}(\theta)}
$$


Now, if we take arbitrary element excitation by re-writing the array factor expression as

$$
A F=I_{0}+I_{1} e^{j k d \cos (\theta)}+I_{2} e^{j k 2 d \cos (\theta)}+\ldots+I_{N-1} e^{j k(N-1) d \cos (\theta)}
$$

where, $I_{m}$ represents the magnitudes of the element signals.

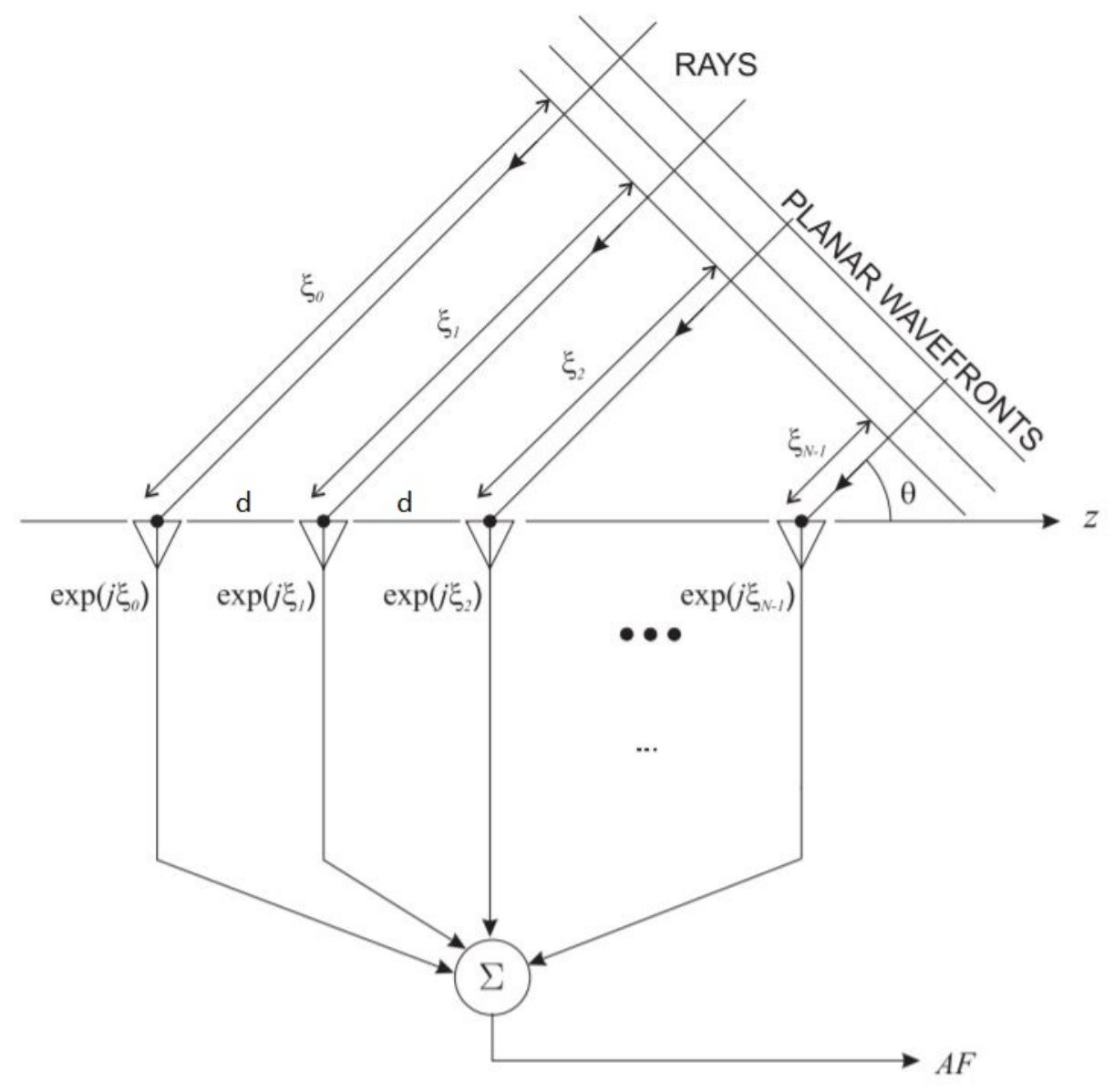

Figure 2.2: Ray diagram for linear receiving array, Reprinted [20]

Now, we consider a phased array that has a circular arrangement. The angle between elements is assumed to be uniform (fig 2.3), such that

$$
\Delta \phi=\phi_{n+1}-\phi_{n}=\frac{2 \pi}{N}
$$




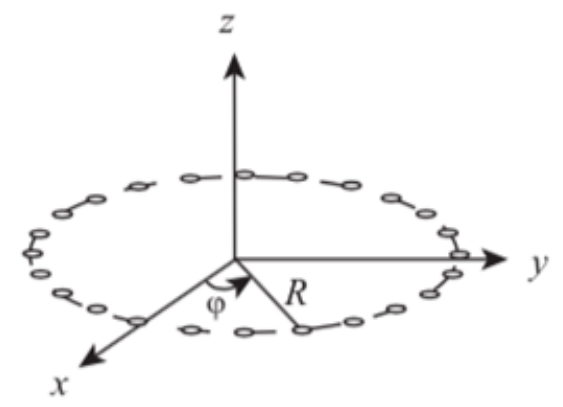

Figure 2.3: Circular array of emitters, Reprinted [31]

If this is the case, the position vector of the nth element can be written as

$$
r_{n}=a \cos \phi \hat{x}+a \sin \phi \hat{y}
$$

where

$$
\phi_{n}=n \Delta \phi=\frac{2 \pi}{N} n
$$

The corresponding array factor expression from eq (2.6) is [20]

$$
A F=\sum_{n}^{N-1} I_{n} e^{j k a\left[\cos \frac{2 \pi n}{N} \sin \theta \cos \phi+\cos \frac{2 \pi n}{N} \sin \theta \sin \phi\right]}
$$

\subsection{Optical Phased Arrays}

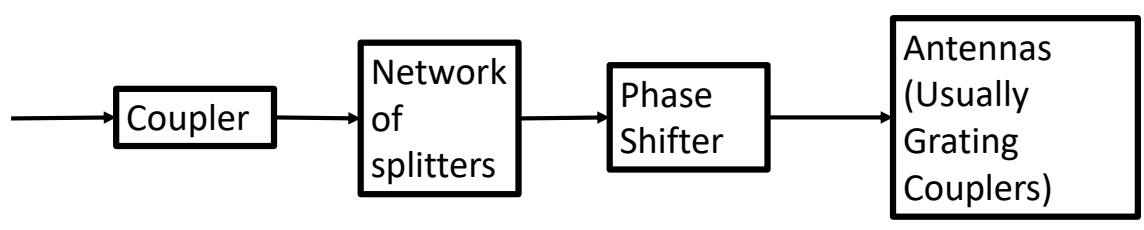

Figure 2.4: Components of a typical OPA 
A typical on-chip OPA includes 4 important sections as shown in Figure 2.4. Usually light is edge coupled into an OPA since it allows polarization independent operation without any complexity in design. Then light is relayed through a network of splitters such as Multimode Interference Couplers (MMIs) or Star couplers [34]. MMI coupler trees can be used for uniform distribution of power in the output. This can be advantageous depending on the type of operation we want from our OPA. Star couplers provide high fan-out at small pitch and provides potential for sub-wavelength pitch. Phase shifters are used to electrically control or program the OPA. Thermo-optic and PN-diode phase shifters are usually used to control the phase of the optical mode. The guided light is then radiated outward by grating couplers. The important performance metrics for an OPA to support free space optical communications are: peak side lobe level, crosstalk, field of vision, phase-shifter bandwidth, angular range, angular resolution, footprint, radiation efficiency and width of radiated beam.

There are two classes of OPAs depending on the type of laser input. OPAs with tunable lasers use the tunability of light's wavelength to steer the emitted beam. They are usually designed with 1D array of grating couplers such as the one in Figure 2.5. For such OPAs the beamwidth scaling is proportional to the number of grating emitters and thus largely simplifies the control electronics. They also have the advantage of large steering range (maximum of $80^{\circ}$ has been achieved) and good angular resolution (minimum beam divergence of $0.14^{\circ}$ ) [21]. The other class of OPAs use a fixed wavelength system where the emitters are arranged in a 2D plane. The amplitude and phase of the 2D array of emitters can be controlled for full dynamic beam shaping (Figure 2.6). This class of OPA can be promising for 3D imaging and holographic purposes.

\subsection{Single Emitter Analysis}

In this project, we are considering diffraction from circular and rectangular aperture for single emitter analysis. For approximating the near field diffraction we use the Fresnel 


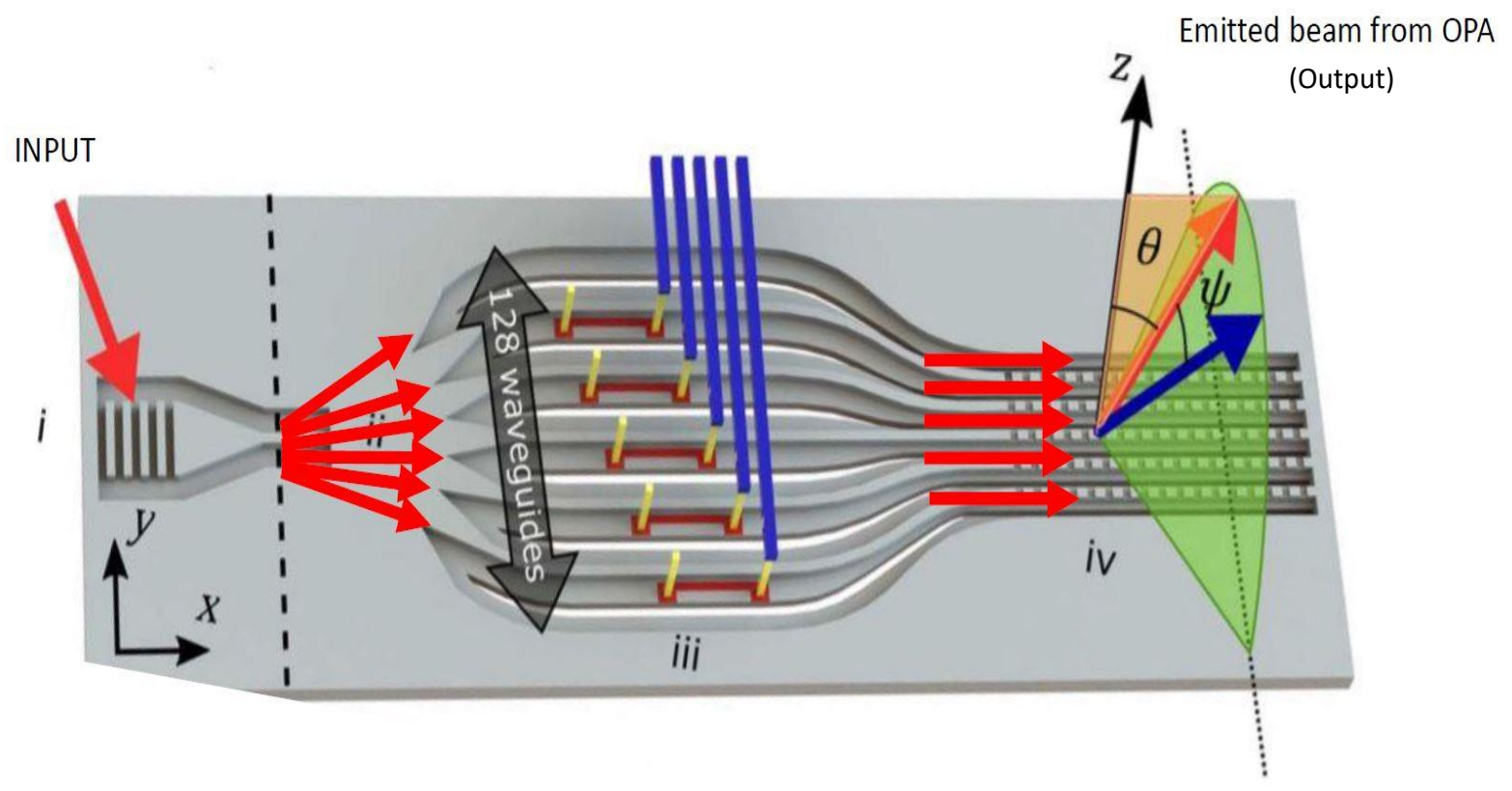

Figure 2.5: OPA with tunable laser source. The phase shifters and tunable laser both are used to steer the output in two directions. Schematic of the device showing only five channels for clarity. The red arrows indicate the direction of light flow. The beam is steerable along the $\theta$ direction using wavelength tuning and the phase shifters are used to steer in the $\psi$ direction. Reprinted [21]

equation. And for farfield we use Fraunhofer diffraction equation. For optical communication we need to prioritize the far field results and thus we use the Fraunhofer equation for modeling the far-field profile.

The diffraction from a circular aperture in the far field produces airy functions or also known as airy rings. The analytical expression for light diffracted from circular aperture [16]:

$$
I(\theta)=I_{0}\left[\frac{2 J_{1}(k a \sin \theta)}{k a \sin \theta}\right]^{2}=I_{0}\left[\frac{2 J_{1}(x)}{x}\right]^{2}
$$

We plot the intensity of light diffracted from circular aperture for different aperture radius: 


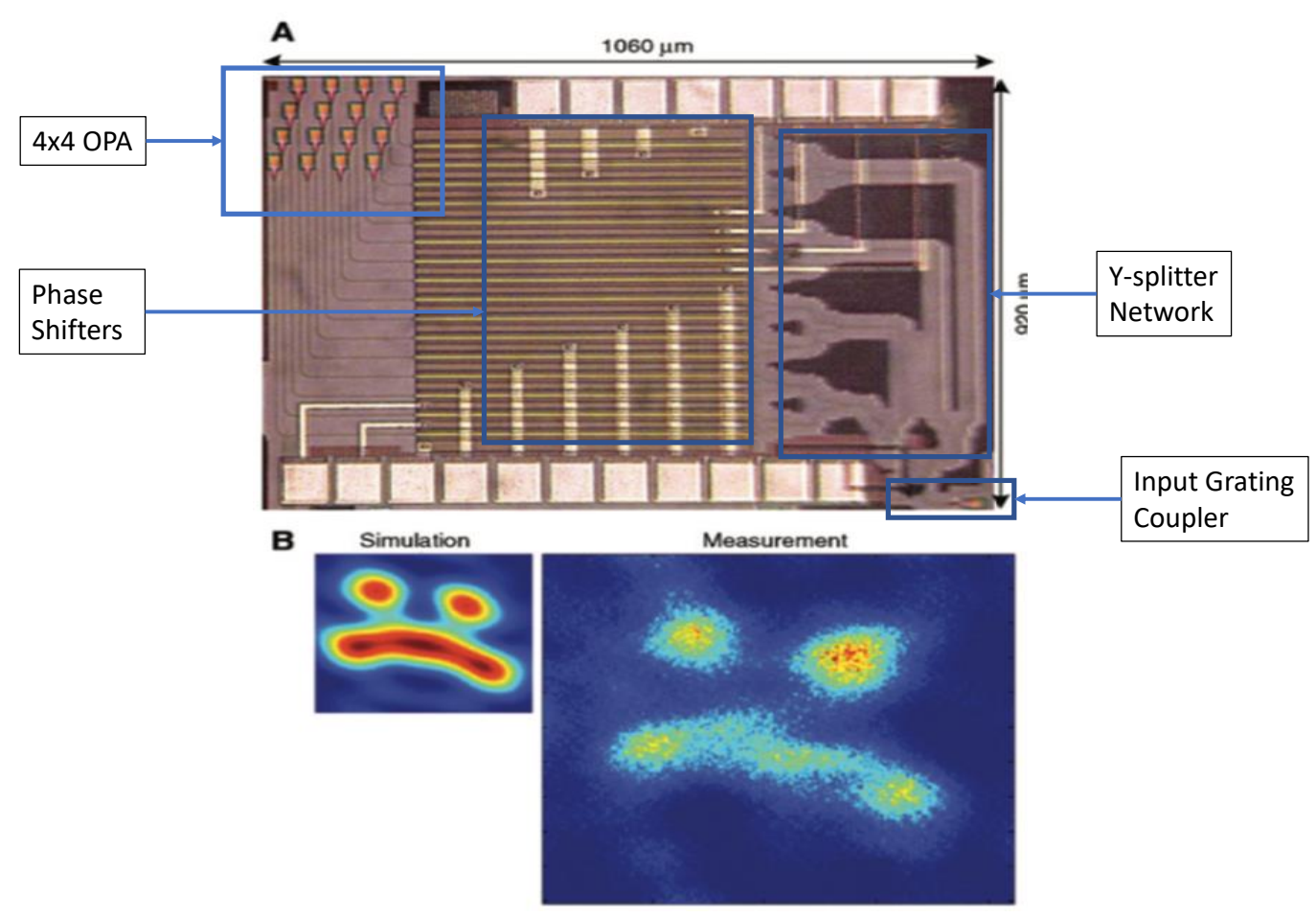

Figure 2.6: A. Chip micrograph of a $4 \times 4$ OPA with fixed wavelength. Light after getting coupled through a grating coupler is guided to a network of Y-splitters. The network is then split into $16 \mathrm{p}$-i-n phase modulators and finally fed into 16 grating couplers. The $4 \times 4$ OPA is designed with a rectangular arrangement. B. The projected far field pattern generating a sad face. Reprinted [1].

Figure 2.7 shows us that, as we increase the aperture radius, the far field profile becomes more narrow. Ideally we want the far-field profile to be as wide as possible because from phased-array point of view that would give us more angular range. But for practicality, 3 um radius is a good choice for aperture radius.

We also did analysis for a rectangular aperture of width $W$ and length $H$, the analytical expression for light diffracted from rectangular aperture [16]:

$$
I(\theta, \phi)=I_{0}\left[\frac{k W \sin \theta}{2} \cdot \frac{k H \sin \phi}{2}\right]
$$




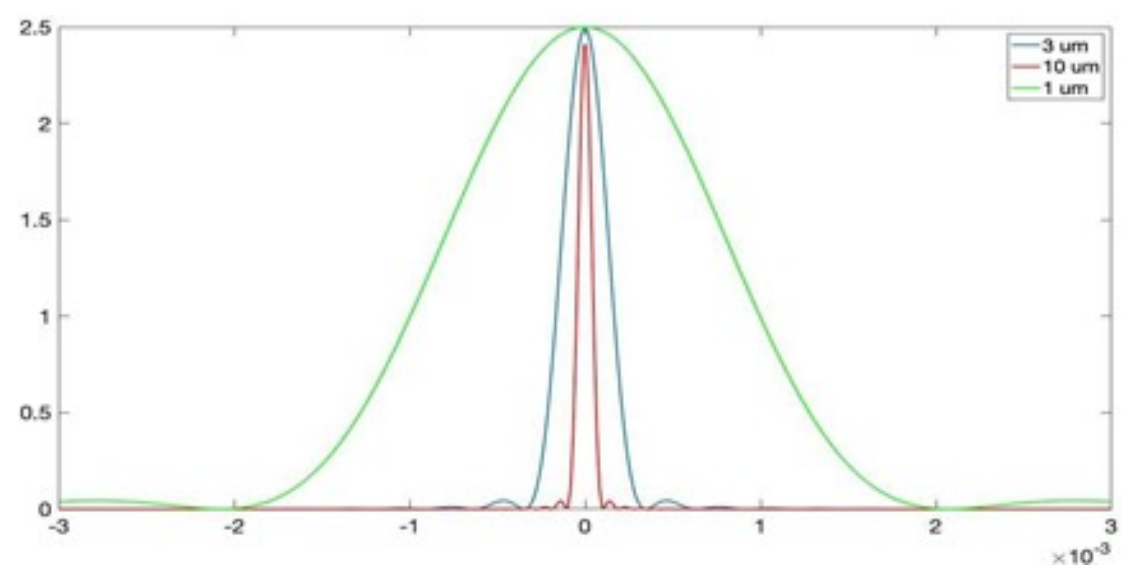

Figure 2.7: $\mathrm{X}$ coordinates $(\mathrm{m})$ vs Intensity $\left(W / m^{2}\right)$

We plot the far field pattern of a $3 \times 9 \mu \mathrm{m}^{2}$ rectangular aperture and the pattern of a grating coupler of same dimensions in Figure 2.8. We use these profiles for our OPA far field analysis.

a)

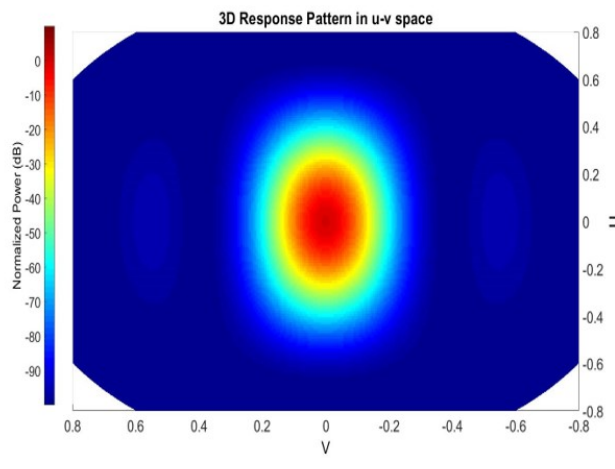

b)

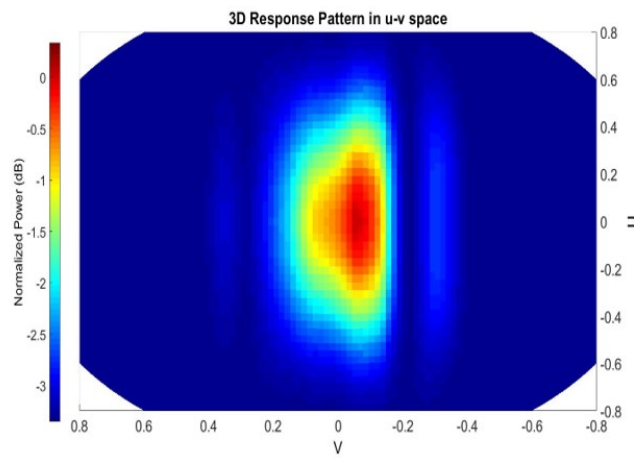

Figure 2.8: Far field pattern of a $3 \times 9 \mu \mathrm{m}^{2}$ (a) rectangular aperture and (b) grating coupler of same dimensions. Here $U=\sin (\theta) \cos (\phi)$ and $V=\sin (\theta) \sin (\phi)$ where $\theta$ and $\phi$ are polar and azimuthal angles respectively. In a spherical coordinate system, the azimuthal angle is defined as the orthogonal projection of a point on a reference plane that passes through the origin and is orthogonal to the zenith, measured from a fixed reference direction on that plane. 


\subsection{Far Field Simulation and Optimization of Antenna lo- cations}

For this project we consider a circularly arranged OPA with two or more rings with 100 emitter elements as shown in Figure 2.9. Circularly arranged OPAs are convenient for space based communications since the circular shaped collimated beam will be compatible with telescope apertures. As reported in literature circularly arranged OPA can provide better sidelobe performance.

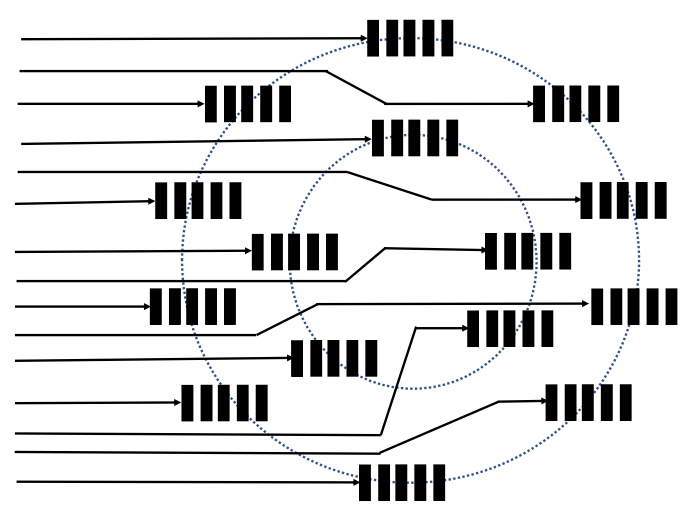

Figure 2.9: Schematic of emitter region of dual ring OPA

Equation 2.10 is used to calculate the far field of a circularly arranged OPA. As can be seen from the equation, we need the phase information of each array element. Delay and sum beamforming algorithm [31] can be used to find the phase information to output a plane wave at a certain angle.

We now take a look at the far-field outputs with different emitter configuration. Figure 2.10 shows the far field output of a dual ring OPA with a total of 100 emitters.

In order to improve the peak sidelobe performance of circularly arranged OPAs we take a look at the far field profile of two single ring arrangements with different radii (Figure 2.11). We see that reducing the radius of the ring will push the sidelobes further away from the main beam. 


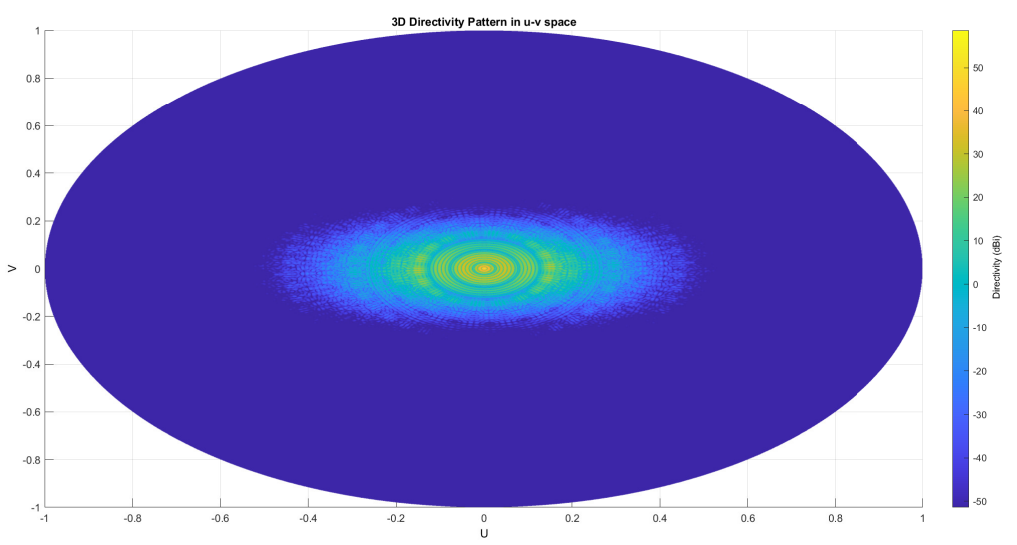

Figure 2.10: Far Field Intensity profile with 100 emitters

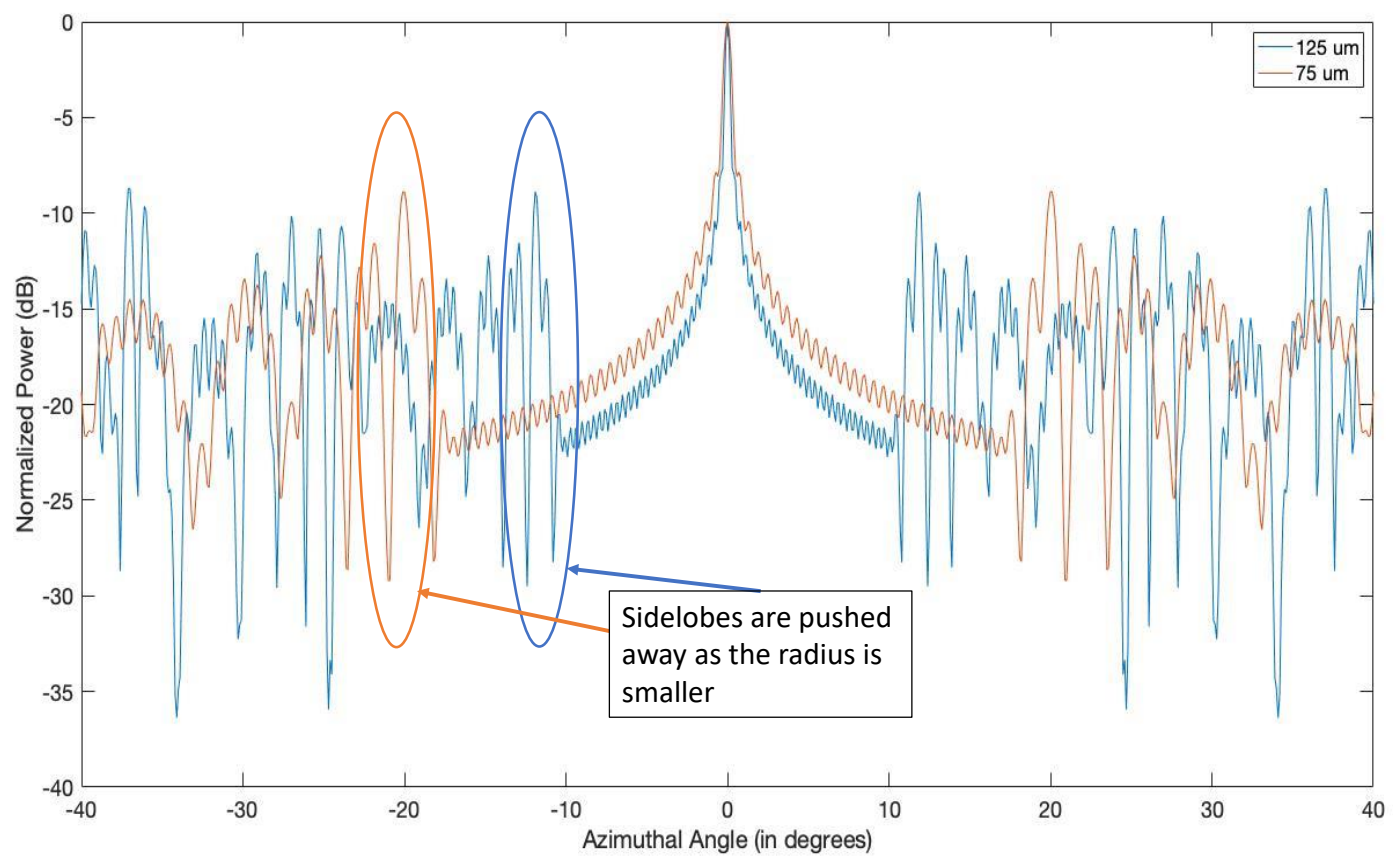

Figure 2.11: Scan of power through 0 degree elevation angle with varying azimuthal angle for single ring OPAs of radii $125 \mu \mathrm{m}$ and $75 \mu \mathrm{m}$

Figure 2.12 shows the far field pattern of dual ring OPA with a fixed inner radius at $43.5 \mu \mathrm{m}$. We see that as we increase the outer ring radius the sidelobes closer to the main beam reduce while sidelobes further away are increased. Thus, for our optimization program we start with deciding the outer ring radius by keeping a fixed inner ring. The 
inner ring is fixed based on our routing abilities taking into consideration the dimension of the grating couplers and the minimum bend radius. For our current optimization problem, we chose $100 \mu \mathrm{m}$ as the After fixing the outer ring, we do a sweep of the inner ring radius as shown in Figure 2.13. We see that for various antenna spacing we have PSLL (in $\mathrm{dB}$ ) below $-13 \mathrm{~dB}$. This gives us a range of options to choose for our OPA design and can decide on the optimal choice for routing the grating couplers.

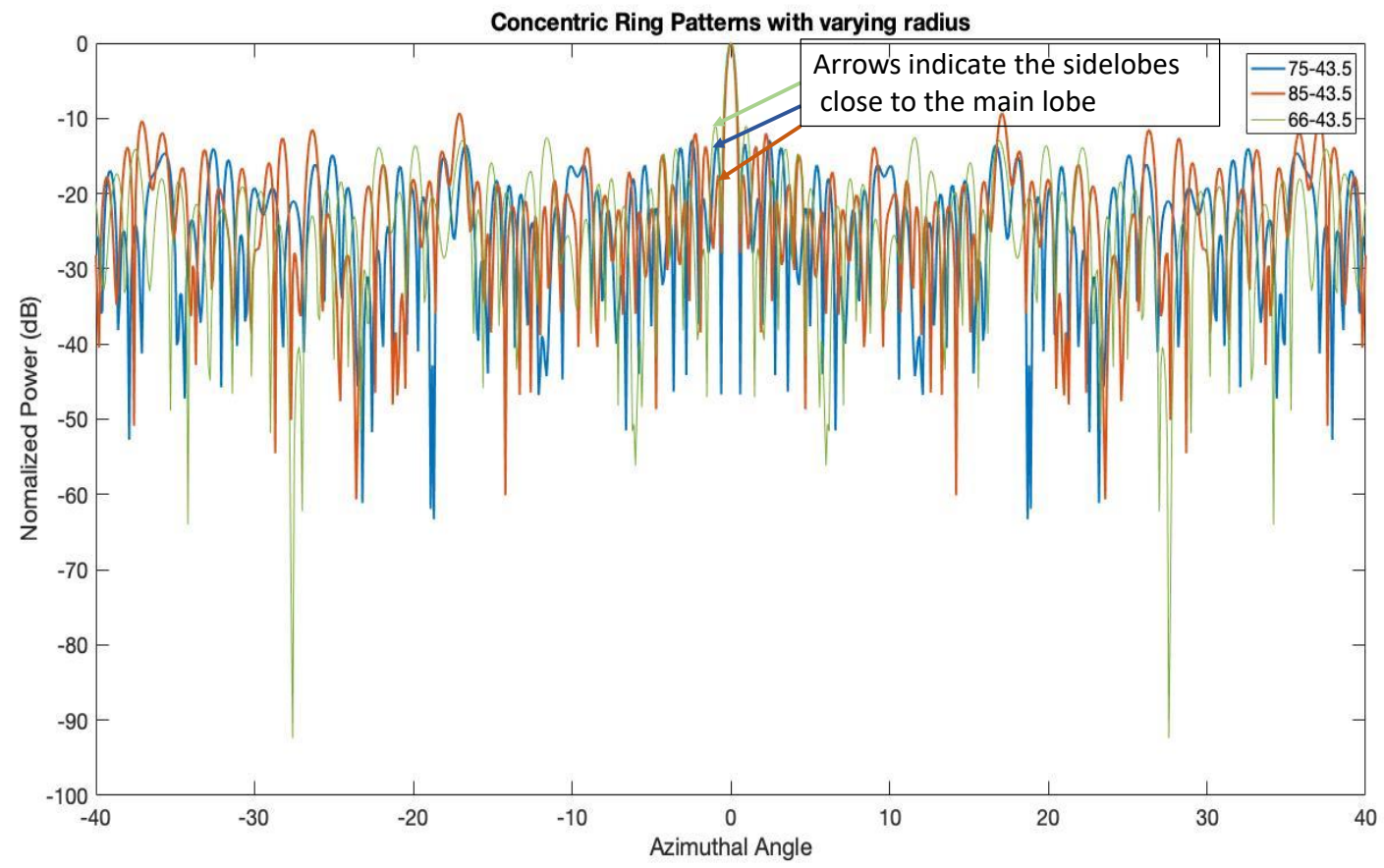

Figure 2.12: Scan of power through 0 degree elevation angle vs azimuthal angle for dual ring OPAs with fixed inner ring radius at $43.5 \mu \mathrm{m}$ and outer ring radii at $66 \mu \mathrm{m}, 75 \mu \mathrm{m}$ and $85 \mu \mathrm{m}$.

We also wrote an optimization algorithm for an $\mathrm{N}$ number concentric rings OPA with a fixed number of total elements (for example: we used 100 elements for our analysis) as shown in figure 2.14. We took the spacing between elements and the radii of the rings as the driving parameters for our optimization. We keep R1 fixed at $75 \mathrm{um}$ and sweep $\mathrm{R} 2, \mathrm{R} 3, \mathrm{R} 4$ and record the lowest peak sidelobe level. Then we sweep $\mathrm{d} 1, \mathrm{~d} 2, \mathrm{~d} 3$ and $\mathrm{d} 4$ keeping in mind the constraint that the total number of elements cannot cross 100 . The 


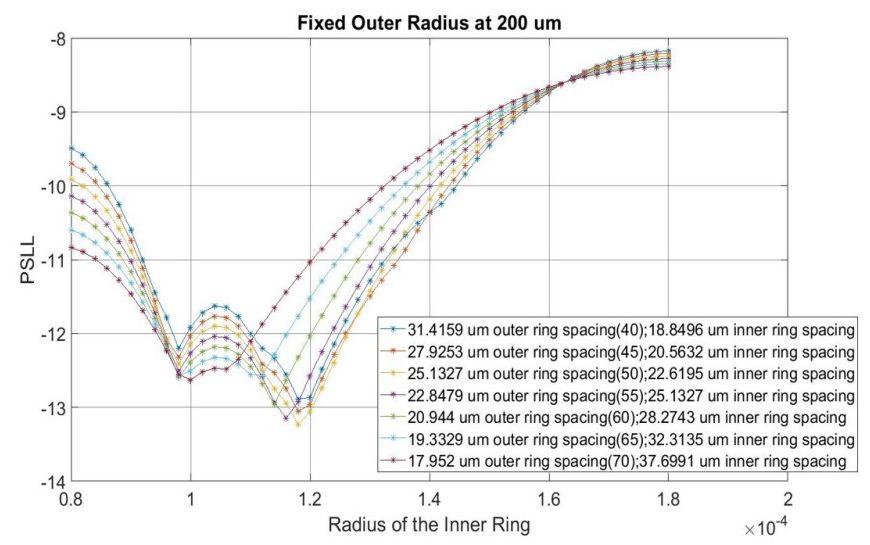

Figure 2.13: Peak Sidelobe level (PSLL in $\mathrm{dB}$ ) vs inner ring radius with outer ring radius fixed at $200 \mu m$

lowest peak sidelobe level we could achieve using four rings is $-15.3 \mathrm{~dB}$ which is around $17 \%$ of the main lobe.

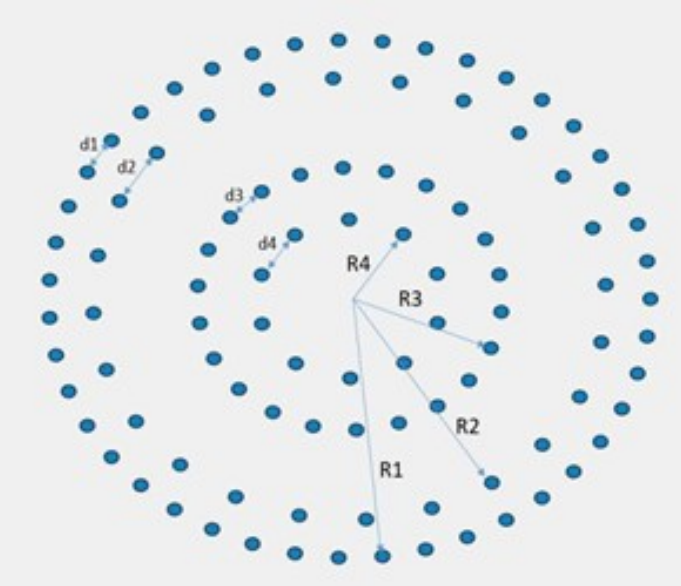

Figure 2.14: Optimizing the element position of four ring OPA

\subsection{Beam Steering Algorithm}

This section focuses on the beam steering algorithm that would be useful if we wish to steer an optical phased array. Figure 2.15 shows a flow-chart of the optimization process presented in [1]. 


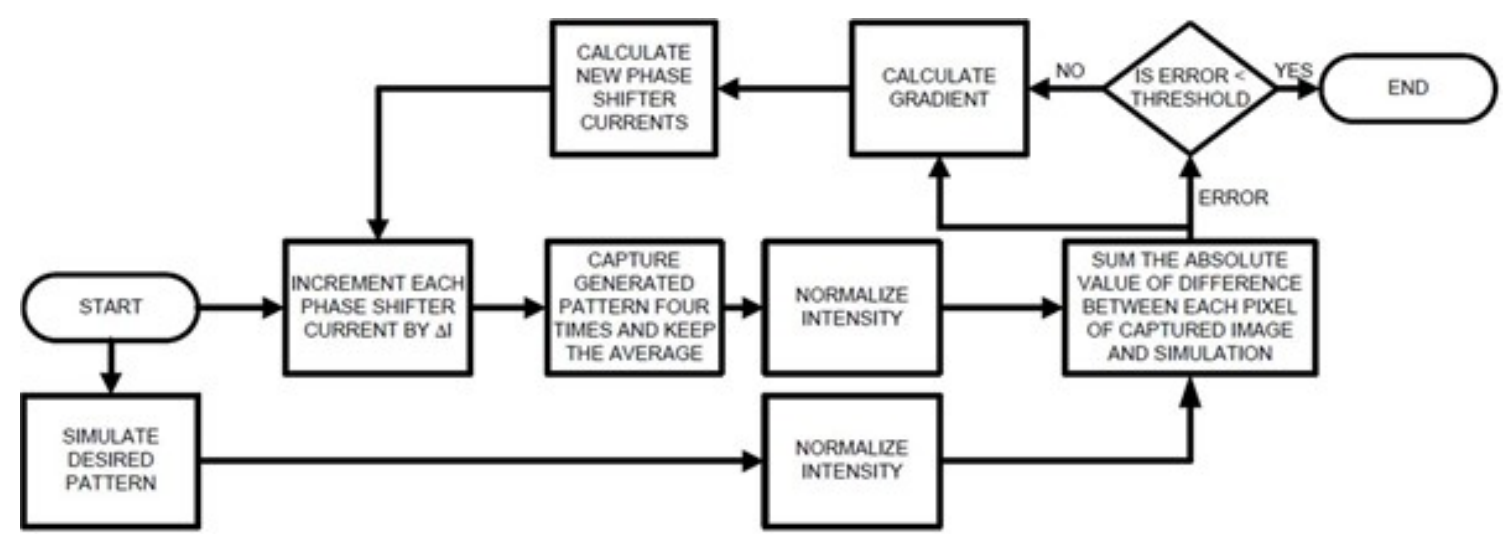

Figure 2.15: Flowchart of the optimization process, reprinted from [1]

In the optimization process, the image captured by a camera corresponding to the main lobe of the phased-array pattern is compared against that of a simulated phasedarray pattern. The error is defined as the pixel-wise Euclidean distance between the simulated pattern and the captured image and then used in the gradient descent optimizer. In each iteration, the gradient of the error is calculated by increasing the current of each phase shifter and observing the changes in the captured pattern as well as the error (For simulation purposes we manually update the phase of each phase shifters). At the end of each iteration, the currents of the phase shifters are modified by a small amount in the opposite direction of the gradient.

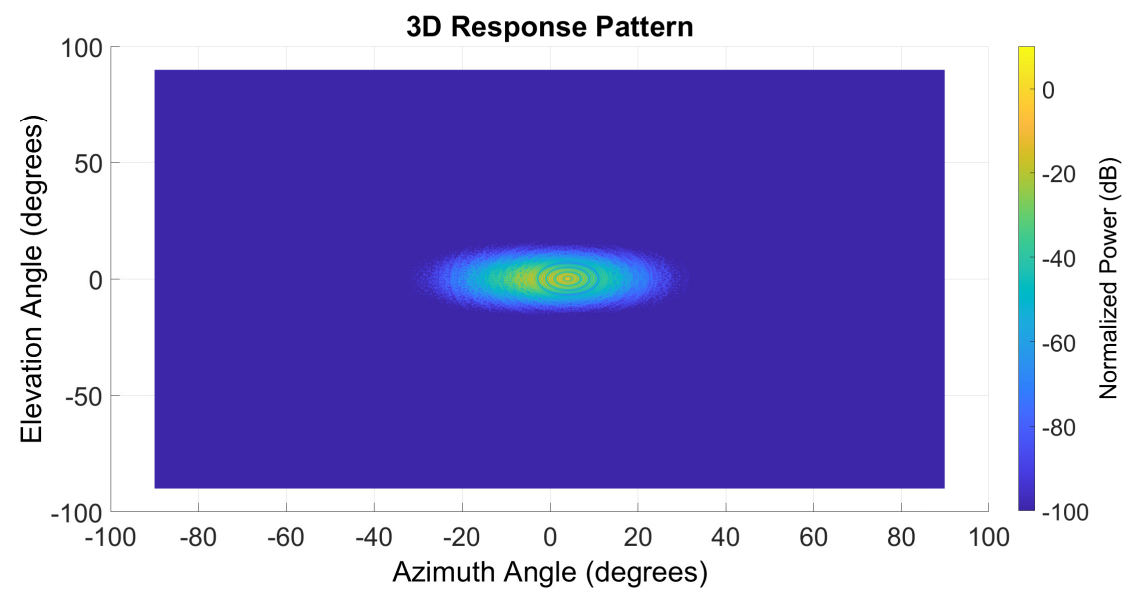

Figure 2.16: Intensity profile of a steered beam at $4^{\circ}$ 
a)

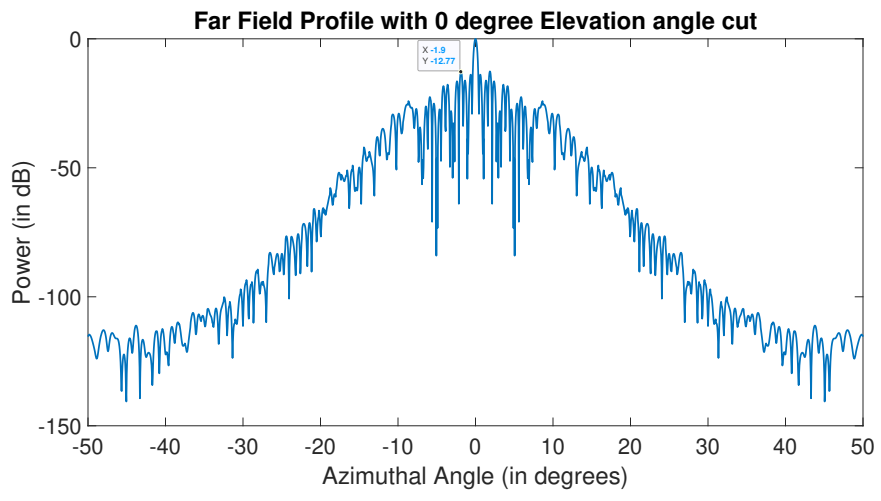

b)

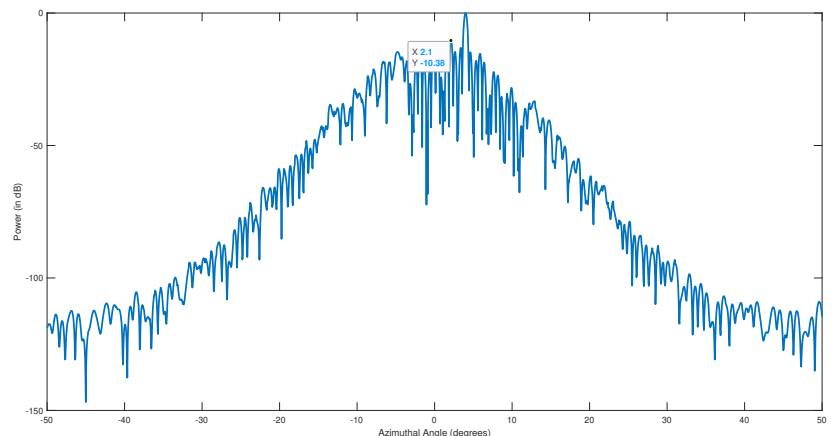

Figure 2.17: Scan of power in $\mathrm{dB}$ vs azimuthal angle for $0^{\circ}$ elevation cut with a) $0^{\circ}$ steering and b) $4^{\circ}$ steering

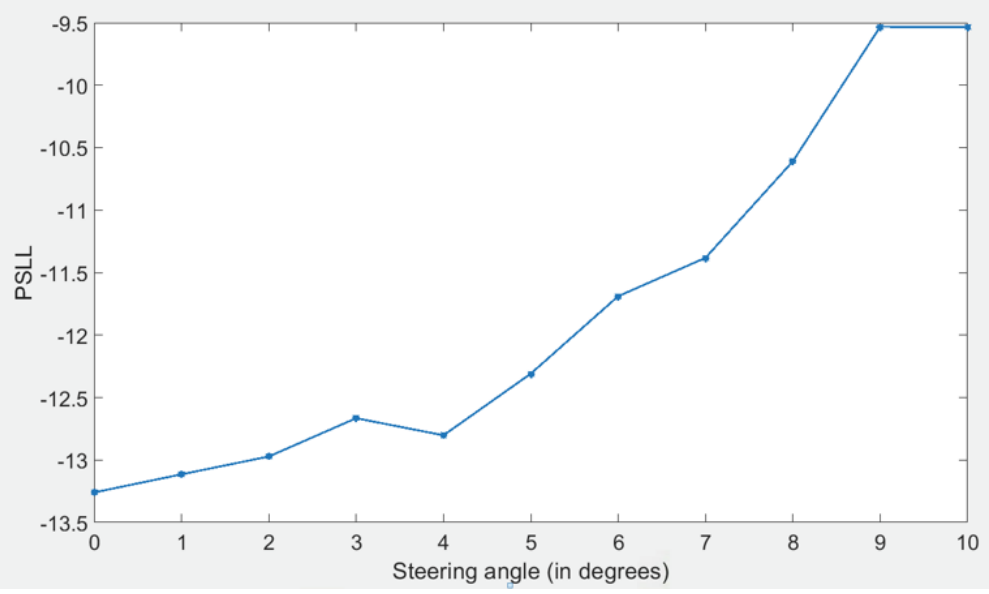

Figure 2.18: Steering angle vs PSLL (in dB)

We simulate the flowchart in Figure 2.15 by calculating the far field output after updating the phase information in equation 2.10. Figure 2.16 shows the intensity profile of a steered beam at $4^{\circ}$. 
From Figure 2.17, we see that the steered beam has a higher sidelobe. Thus, we can calculate the highest steering range where the PSLL has to be kept below a certain level. Figure 2.18 shows the PSLL (in $\mathrm{dB}$ ) vs the steered angle for a 100 element dual ring OPA with $55 \mu m$ inner ring and $75 \mu m$ outer ring OPA.

\subsection{Closed-loop phased-array control algorithm}

In the previous section, we described a gradient descent approach for beam steering. One disadvantage of this approach is that it requires the evaluation of a large number of intensity patterns to obtain the desired phase information. Thus, evaluation and gradient optimization requires huge time and computing resources. Figure 2.19 shows the flowchart of our proposed closed loop control algorithm that performs computation in the frequency domain to control our OPA.

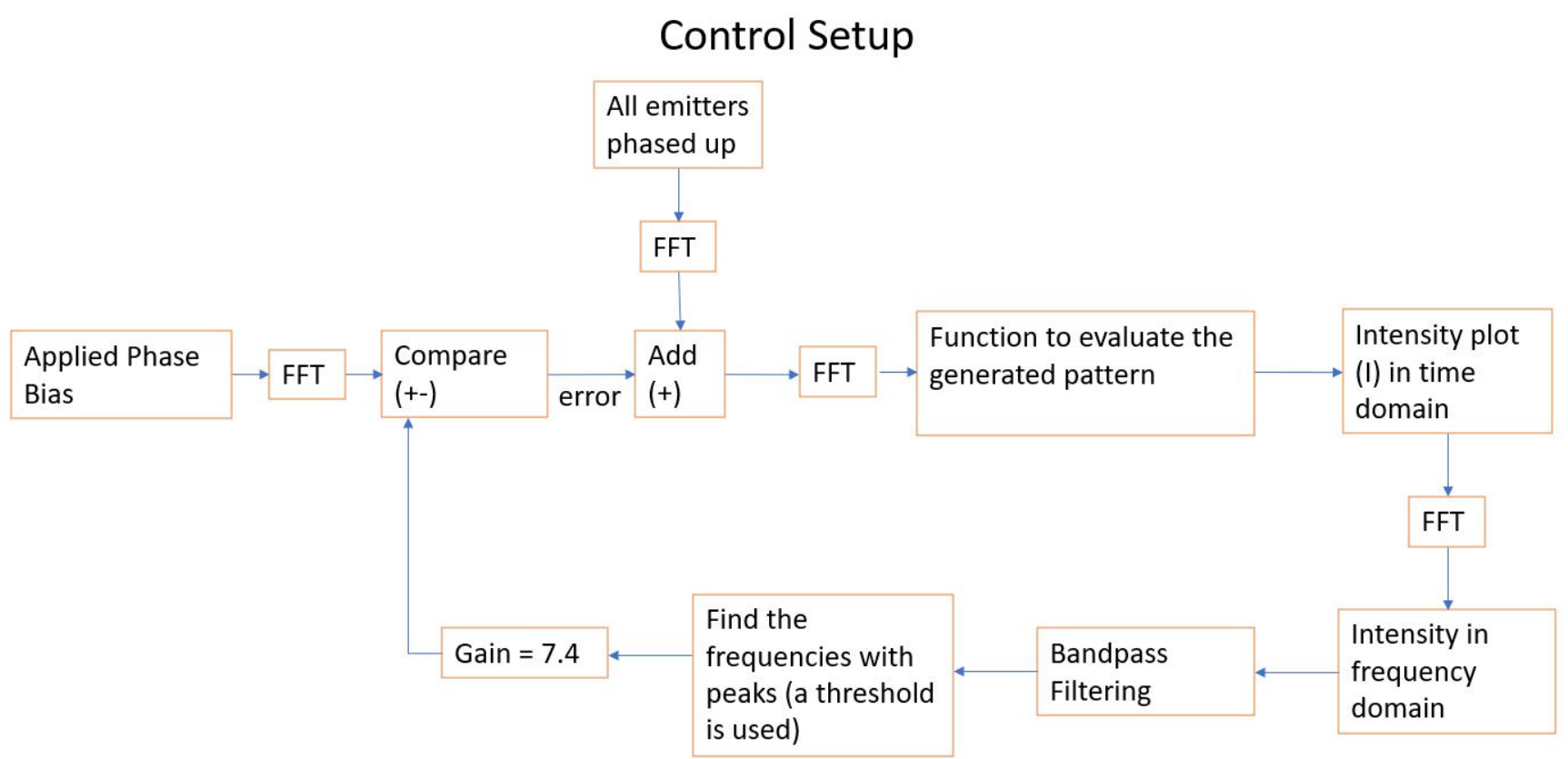

Figure 2.19: The proposed control algorithm

We walk through our control algorithm by simulating a 100 element OPA with dual ring configuration. First, we start with phasing up all the emitters of the OPA with a 


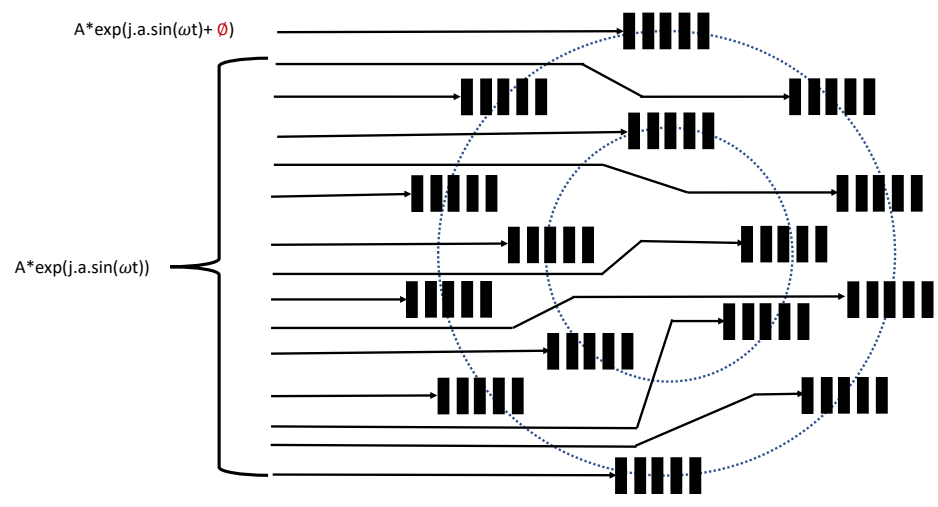

Figure 2.20: The phase of all the emitters are modulated with a sinusoid signal. One of the emitters have a certain phase error. This phase error can be traced in the fourier spectrum.
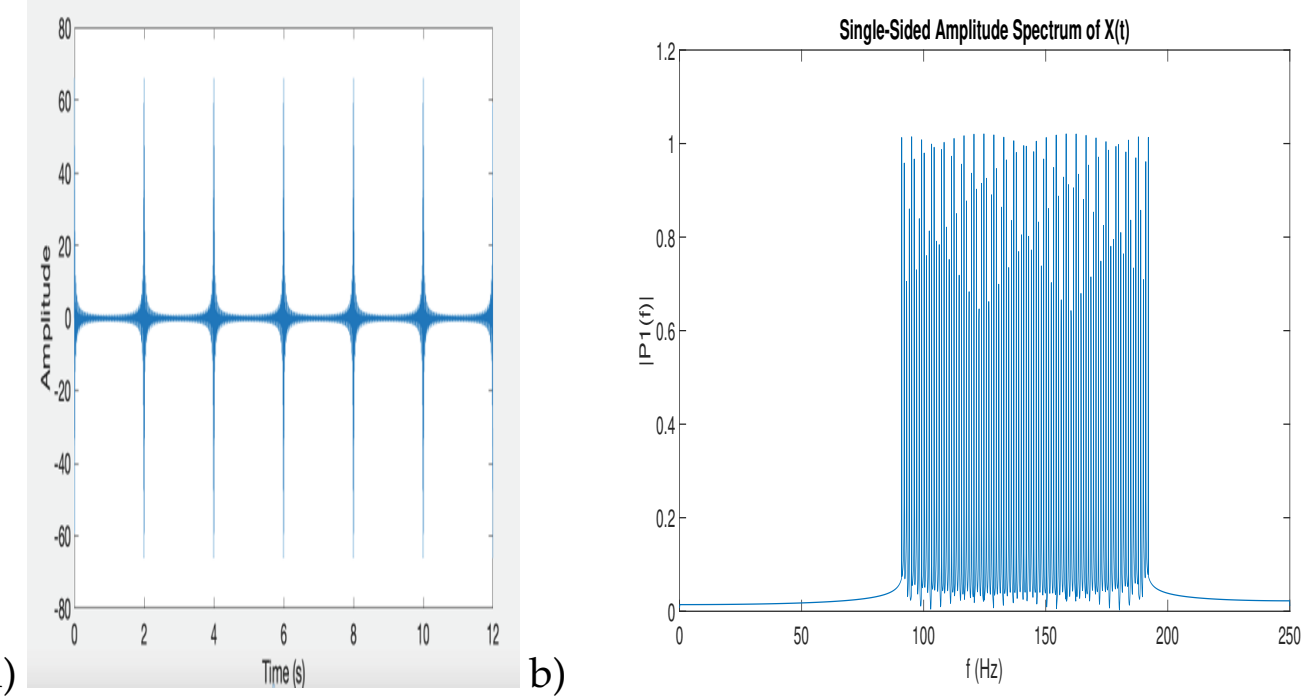

Figure 2.21: a) Time domain and b) Frequency domain signal that acts as an input into the 100 element OPA. All the emitters are modulated with frequencies between $92 \mathrm{~Hz}$ to $191 \mathrm{~Hz}$ with $1 \mathrm{~Hz}$ seperation.

certain amount of bias as shown in Figure 2.20. We modulate the phase of all of the emitters by $a \cdot \sin (\omega t)$ where $a=\pi$. Figure 2.21 shows the input signal in frequency and time domain. The emitters are modulated with frequencies between $92 \mathrm{~Hz}$ to $191 \mathrm{~Hz}$ with $1 \mathrm{~Hz}$ separation. Equation 2.10 can be used to calculate the far field intensity pattern with the current phase information we have and obtain the amount of power in the main lobe for different time values. We simulate for a duration of $14 \mathrm{~s}$ time with $500 \mathrm{~Hz}$ of 


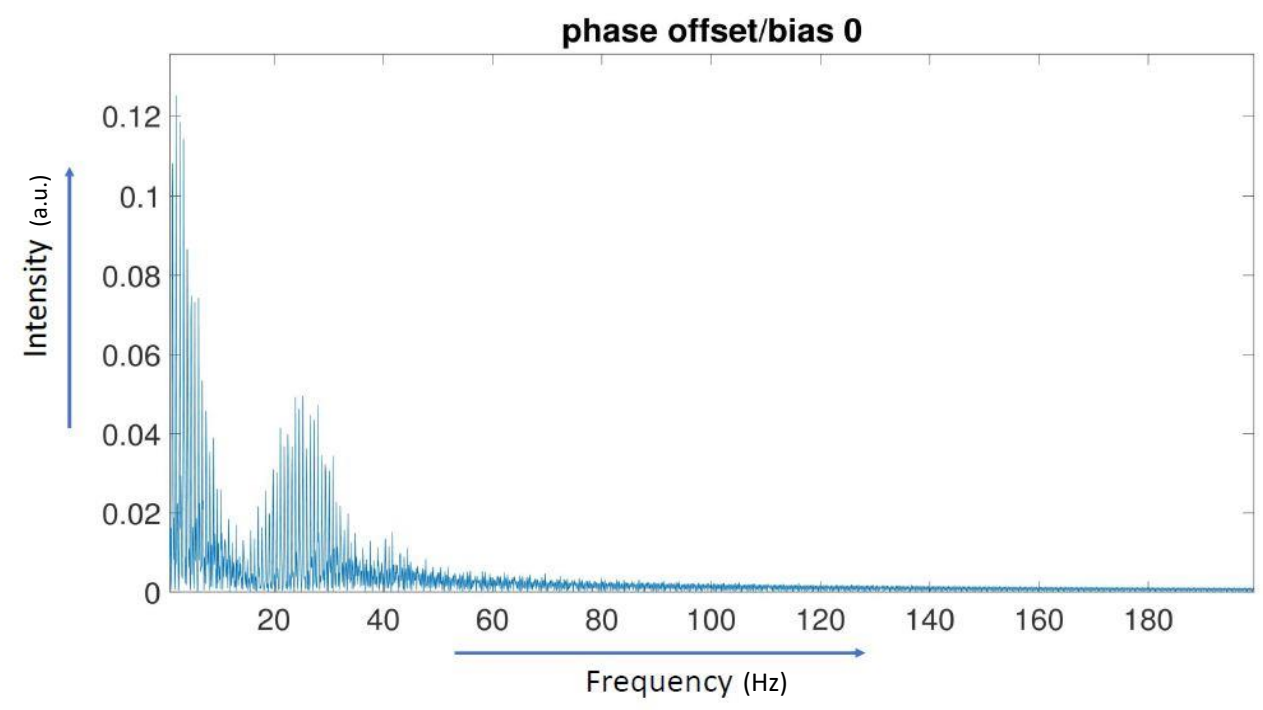

Figure 2.22: Frequency domain output (in $\mathrm{Hz}$ ) of intensity pattern with all of the emitters modulated with a sinusoid signal (No bias applied at any of the emitters). We can see that there are no peaks between $92 \mathrm{~Hz}$ and $191 \mathrm{~Hz}$.

sampling rate. The generated pattern in time domain is then fast fourier transformed into the frequency domain and we can see the results in figure 2.22. We see that there are no peaks between $92 \mathrm{~Hz}$ to $191 \mathrm{~Hz}$, the frequencies with which we have modulated the phase shifters. The interference pattern appears at frequencies double the modulated signals.

We now look at the output spectrum with one emitter with extra bias applied to its phase. Figure 2.23a) shows that as we apply an extra bias of $\phi=15^{\circ}$ to the emitter that is modulated at $191 \mathrm{~Hz}$, we see an intensity peak at the same frequency in our intensity spectrum. Figure 2.23b) presents a case with 4 emitters with $\phi=45^{\circ}$ and one with $\phi=15^{\circ}$. This shows that the magnitude of intensity peak appearing at the output is dependent on the amount of bias applied.

Now, we go back to our flowchart in figure 2.19. Once the peaks are obtained, they are compared in the frequency domain with the desired intensity pattern. For the purpose of our simulations we designed a simple proportional control loop that drives down the phase error of all the emitters. We see that, with a gain of 7.4 applied to the intensity spectrum, we can drive down any phase errors of the system. We can perform beam steer- 


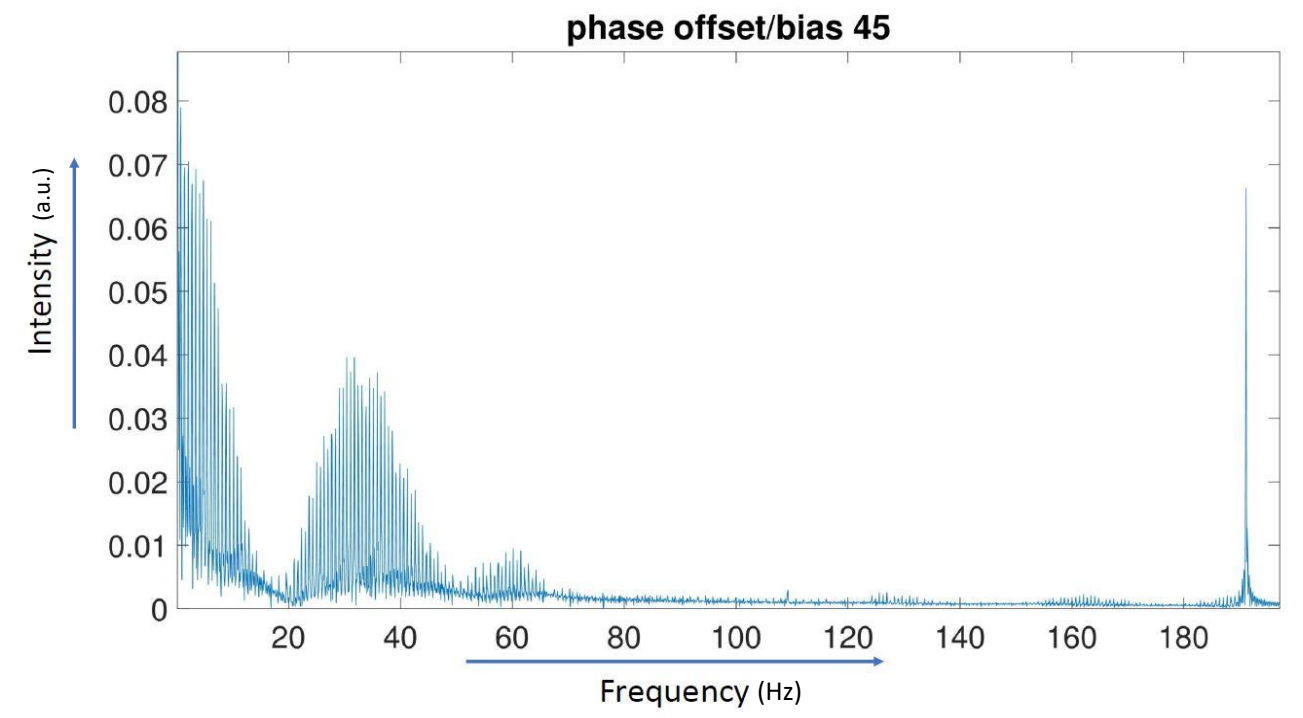

a)

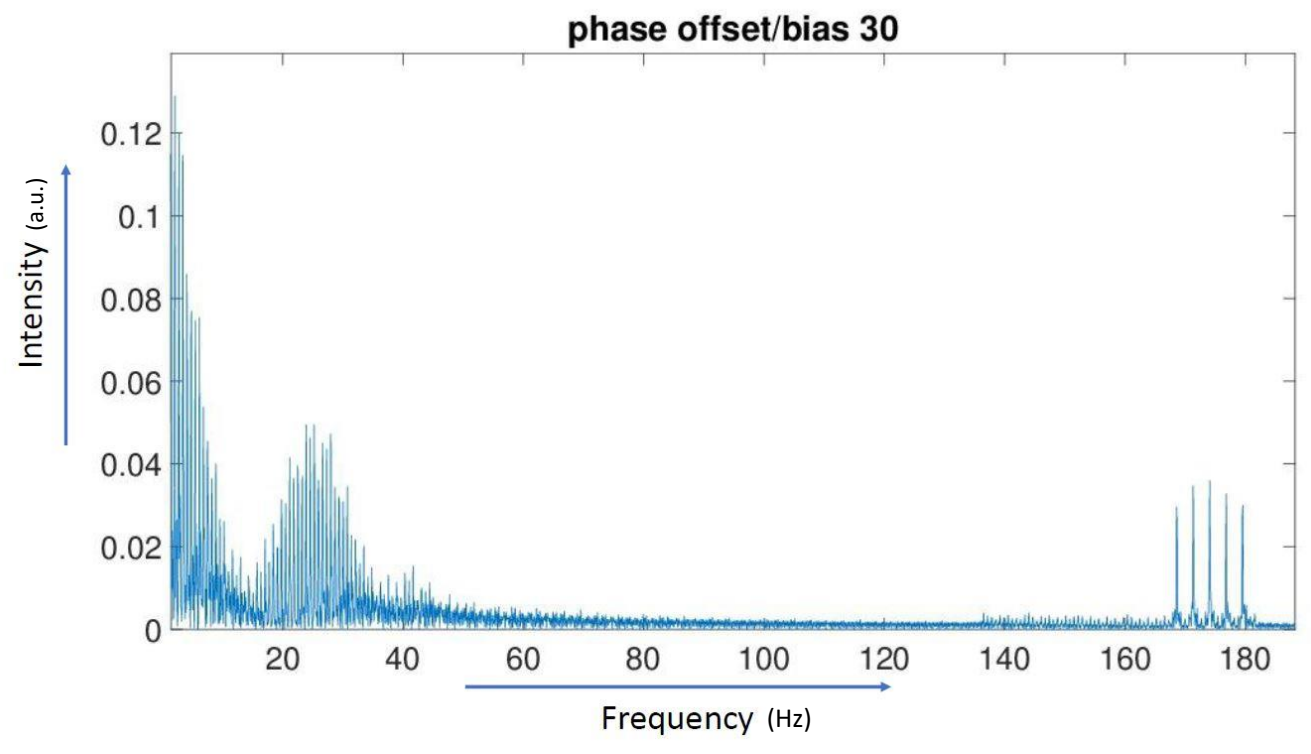

Figure 2.23: Frequency domain output (in $\mathrm{Hz}$ ) of intensity pattern with all of the emitters modulated with a sinusoid signal with a) The emitter with $191 \mathrm{~Hz}$ has extra $45^{\circ}$ phase bias b) Four emitters are at $30^{\circ}$ phase bias. The four emitters are modulated at 168, 172, 176 and $180 \mathrm{~Hz}$. We see the peaks in Fourier domain with a reduced intensity in comparison to $45^{\circ}$ phase bias.

ing using a similar control strategy as well. This approach saves a significant amount of computation cost since it requires fewer function evaluations in the loop. For our control algorithm, we required a total function evaluation of 4 times whereas a gradient descent based approach would require more than 30 evaluations. 


\subsection{Experimental Setup and Results}

We perform experiments with an 8 element circularly arranged OPA provided from Honeywell Aerospace. A microscopic image of the OPA chip is shown in Figure 2.24. The circular arrangement has a radius of $4 \mu \mathrm{m}$ with an overall footprint of $7.6 \mathrm{~mm} \times 1.2 \mathrm{~mm}$. The OPA has $3 \mathrm{~mm}$ long thermo-optic heater deposited above $\mathrm{SiN}$ waveguides with thermooptic coefficients of $2.45 \times 10^{-5}$. We can use equation (1.5) to calculate the amount of phase change that can be achieved for a $3 \mathrm{~mm}$ long Au heater that dissipates $200 \mathrm{~mW}$ of power. Figure 2.25 shows the schematic of our experimental setup to characterize our OPA. A tapered fiber is edge coupled to the OPA with the help of a 6-axis stage. The tapered fiber feeds $780 \mathrm{~nm}$ light into the chip and the emitted light is seen through a zoom stereomicroscope. A Lumenera camera captures the OPA output and the images are then used for data processing. We used National Instruments Data Acquision (DAQ) card (NI 9264) and voltage controlled current source to control the phase shifters. NI 9264 has 16 analog output channels with nominal voltage range of $0-10 \mathrm{~V}$ and maximum current drive of 4 $\mathrm{mA}$ per channel. For our phase shifters we need at least $16 \mathrm{~mA}$ of current to achieve $\pi$ phase shift. Figure 2.26 shows the circuit diagram of the voltage controlled current source that we used to control the phase shifter. We could drive the phase shifters upto $64 \mathrm{~mA}$ of current. Our experimental setup and OPA output are shown in figure 2.27.

As presented in previous section, we modulated all the phase shifters with some of them under constant bias. We could see the effect in the frequency domain. We performed our experiment with 4 different cases and the results are shown in Figures 2.28 and 2.29. From figure 2.28a) we see that when four emitters are modulated with 4, 5, 6 and $7 \mathrm{~Hz}$ with the $7 \mathrm{~Hz}$ element under $2 \mathrm{~V}$ bias we see an intensity peak pop up around $7 \mathrm{~Hz}$ in the frequency spectrum and in 2.28b) we see that as we reduce the amount of bias to $0.5 \mathrm{~V}$ the

peak becomes less visible. Figure 2.29a) and 2.29b) shows two cases where 3 emitters are modulated and we can pinpoint the element under bias in the Fourier spectrum. All these results provide verification that we can identify the emitters with applied phase bias from 


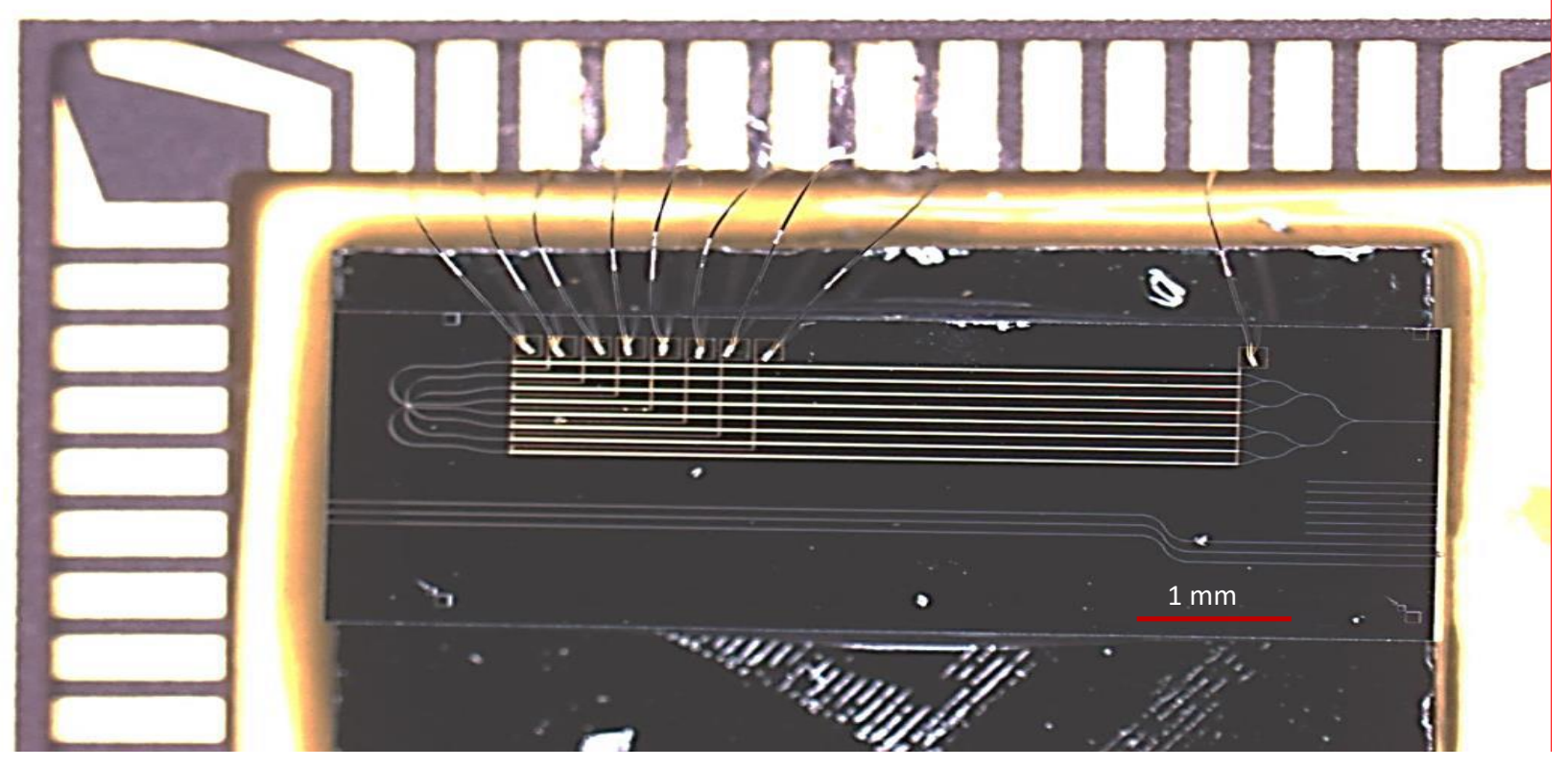

Figure 2.24: Micrograph of the 8 element OPA provided by Honeywell

the frequency domain analysis and the strength of the peaks determine the amount of bias applied. These two important findings provide justification of our proposed control algorithm presented in Figure 2.19.

\subsection{Summary}

In this chapter, we explored the basics of phased array and derived the array factor equation for circularly arranged phased array. We described a method to optimize the positions of circularly arranged OPAs. We proposed a control algorithm to drive down the phase bias of an OPA and compared our algorithm with more popular gradient descent algorithm. 


\section{GSE setup Diagram}

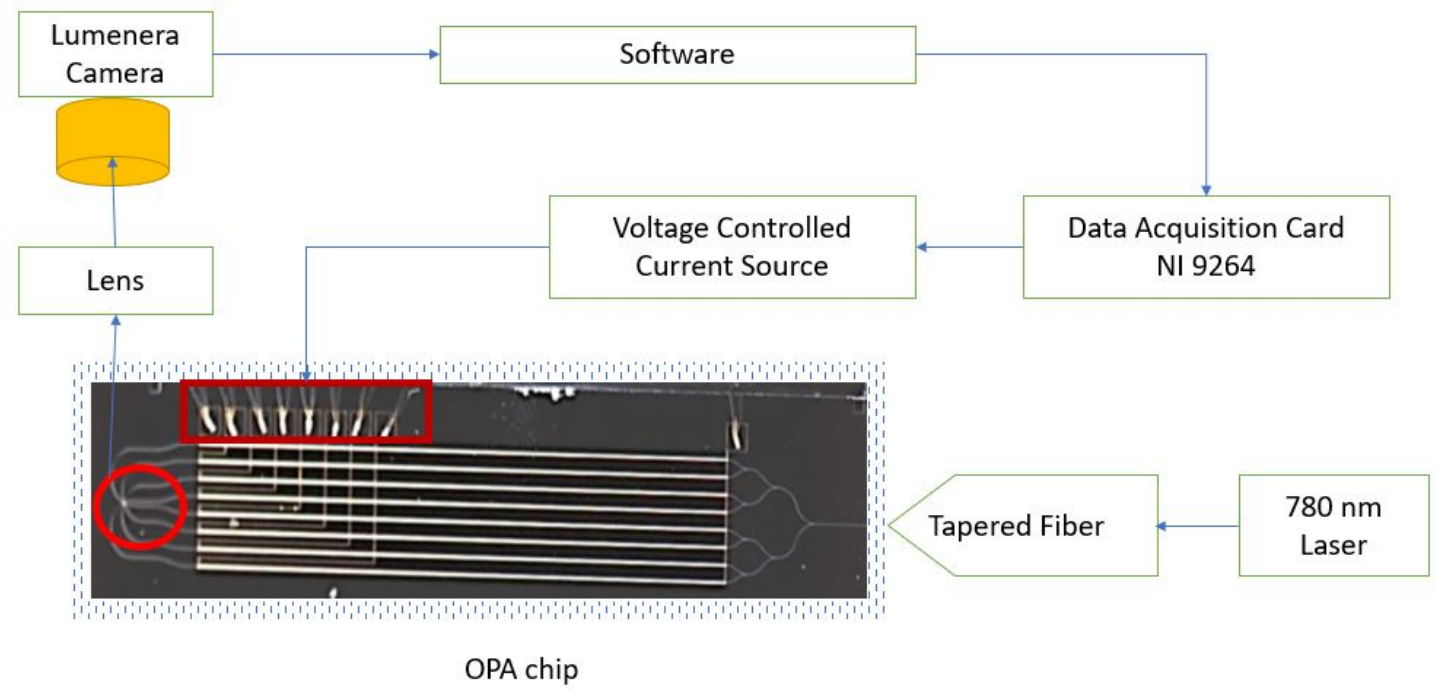

Figure 2.25: Schematic of Ground Station Equipment (GSE) Setup

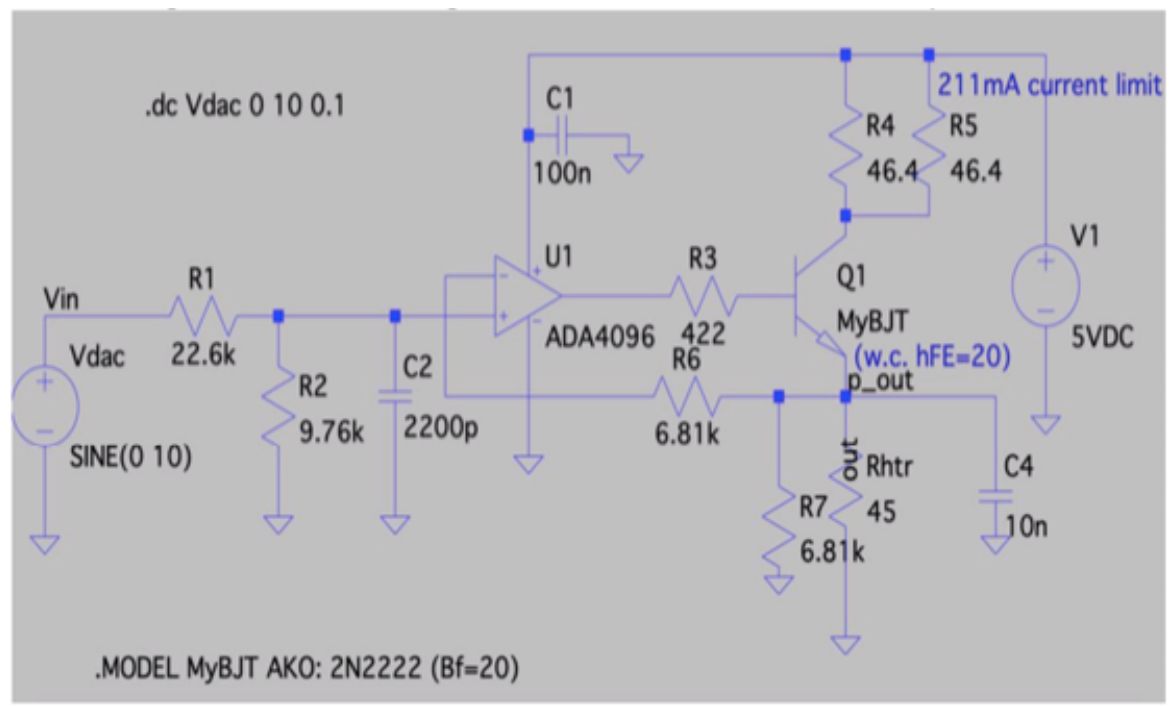

Figure 2.26: Circuit diagram of voltage controlled current source to drive the phase shifters 

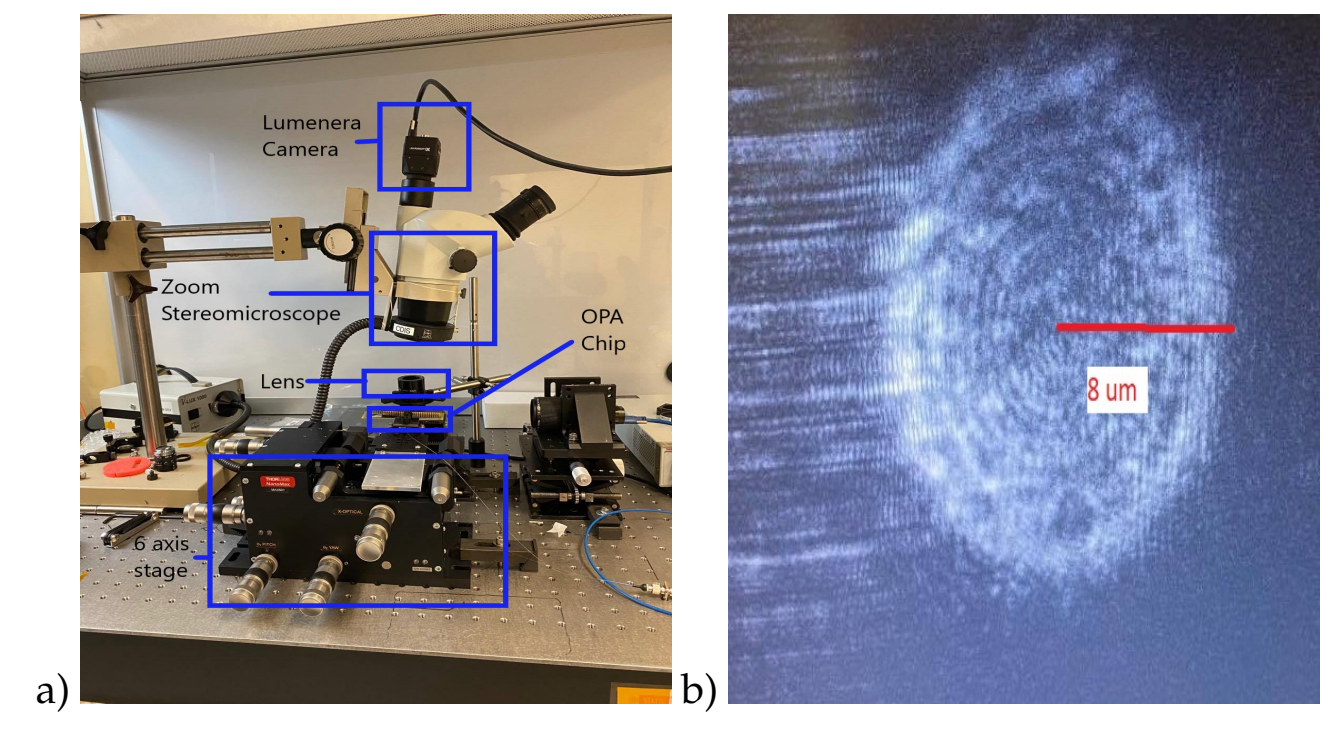

Figure 2.27: a) Photo of our experimental setup for OPA chip characterization, b) Output signal seen from a lumenera camera 


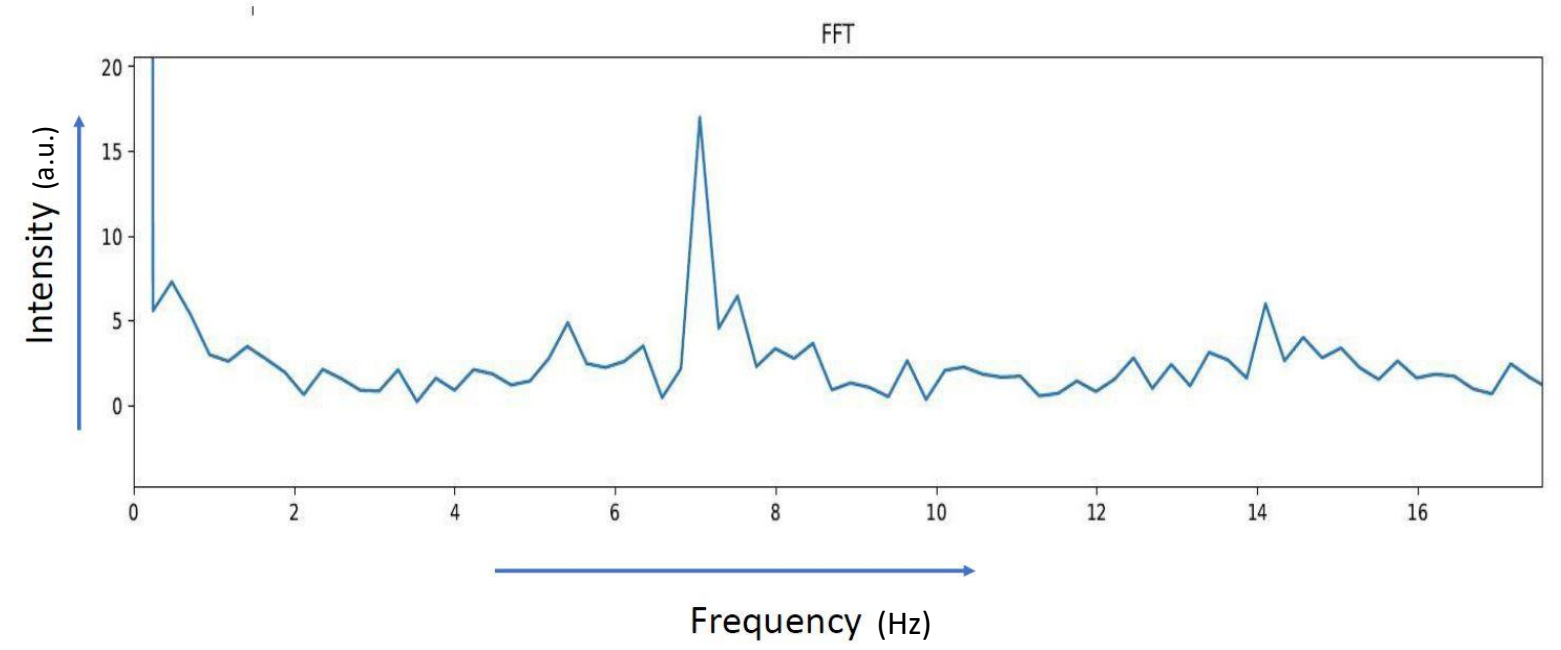

(a) $4 \mathrm{x}$ emitters modulated with 4, 5, 6 and $7 \mathrm{~Hz}$ with the $7 \mathrm{~Hz}$ element under $2 \mathrm{~V}$ bias

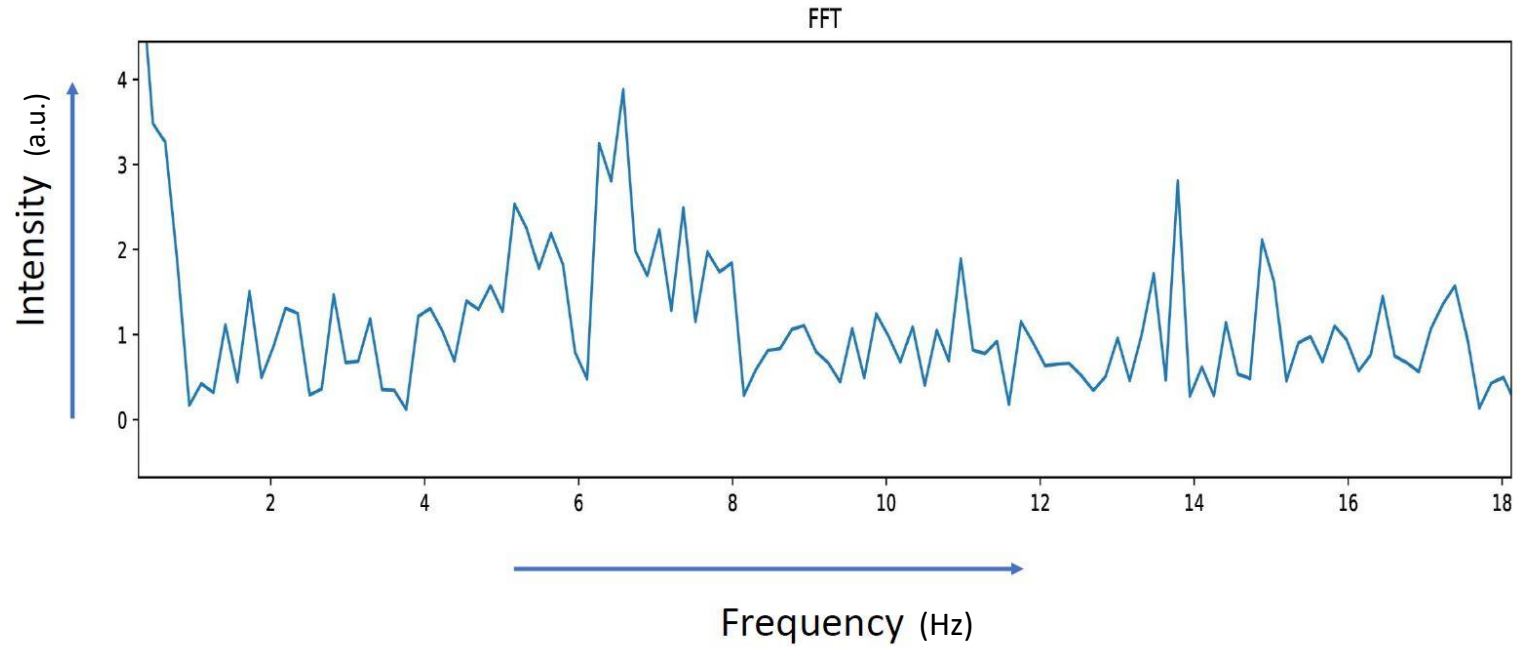

(b) 4x emitters modulated with 4, 5, 6 and $7 \mathrm{~Hz}$ with the $7 \mathrm{~Hz}$ element under $0.5 \mathrm{~V}$ bias

Figure 2.28: Experimental results of 8 element OPA where four emitters are modulated with frequencies $4 \mathrm{~Hz}, 5 \mathrm{~Hz}, 6 \mathrm{~Hz}$ and $7 \mathrm{~Hz}$. The emitter with $7 \mathrm{~Hz}$ has a) $2 \mathrm{~V}$ and b) $0.5 \mathrm{~V}$ constant bias. The upper graphs are plotting the summation of the pixel values of the OPA region vs number of frames. The lower graphs are showing the values in the frequency spectrum. 


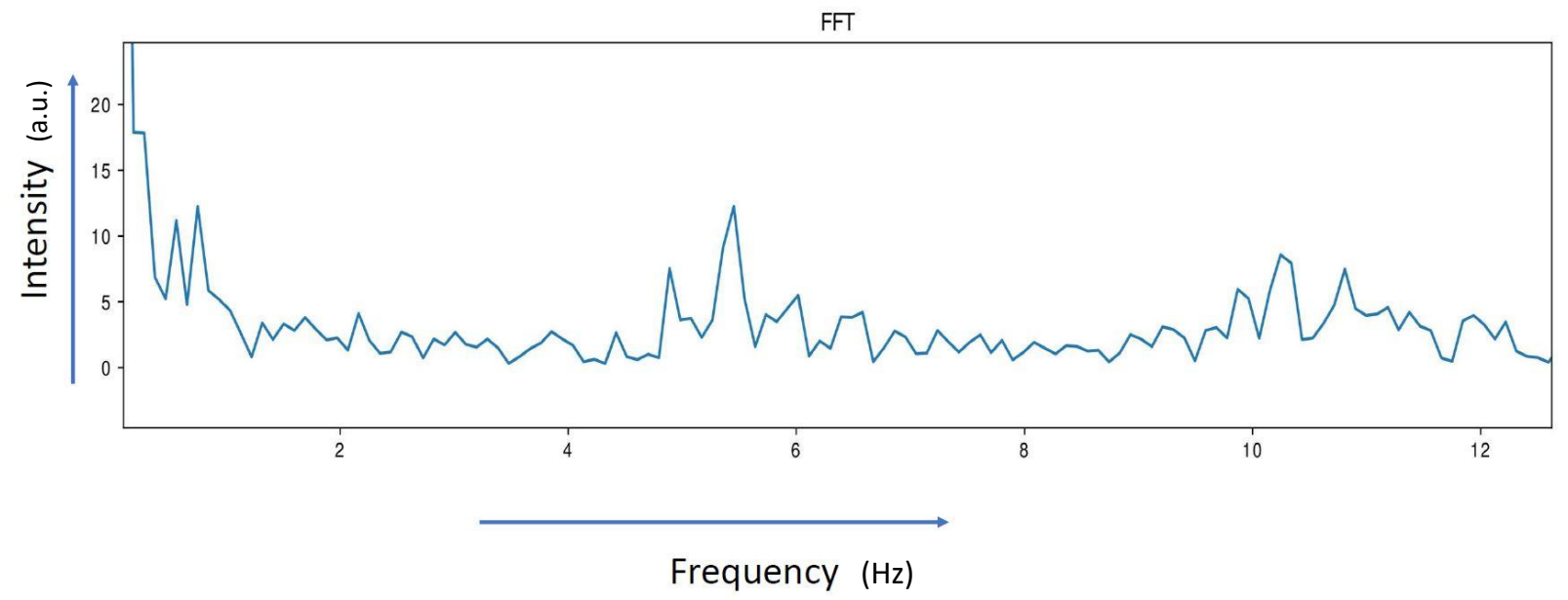

(a) 3x emitters modulated with 4,5 and $6 \mathrm{~Hz}$ with the $5 \mathrm{~Hz}$ element under $2 \mathrm{~V}$ bias

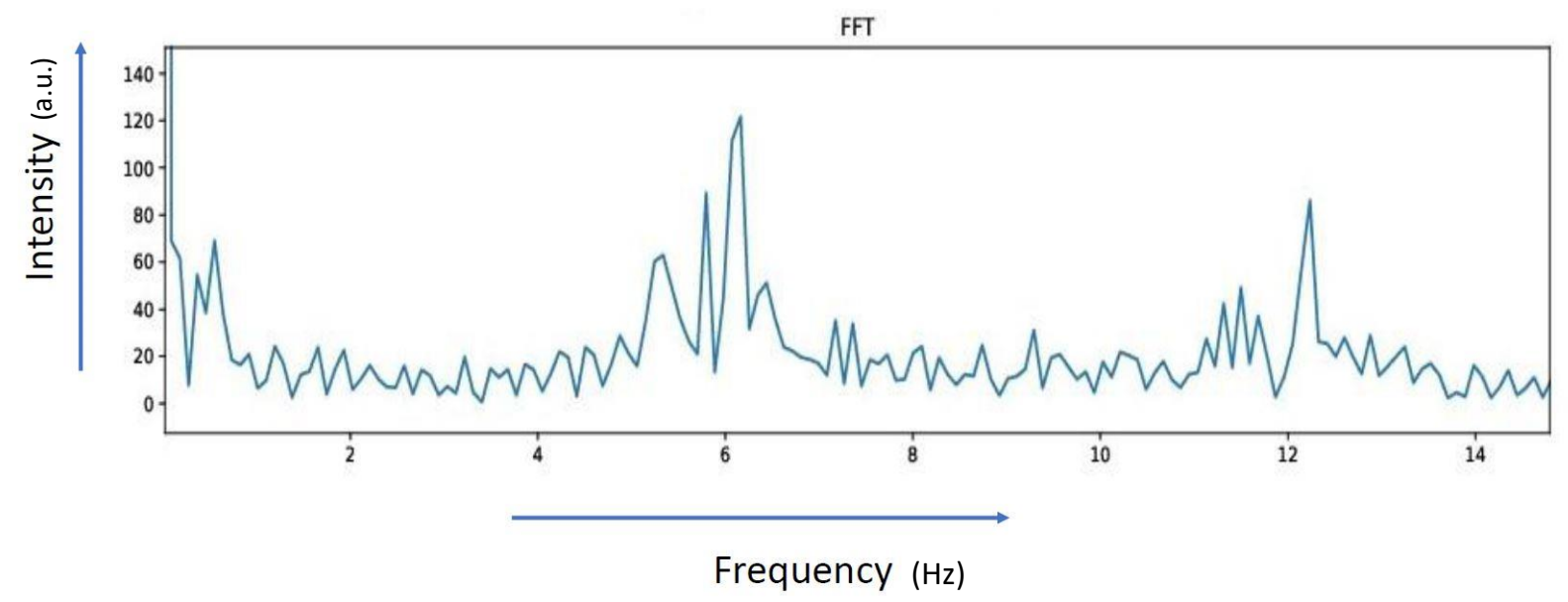

(b) 3x emitters modulated with 5,6 and $7 \mathrm{~Hz}$ with the $6 \mathrm{~Hz}$ element under $2 \mathrm{~V}$ bias

Figure 2.29: Experimental results of 8 element OPA where three elements are modulated. The element under bias can be detected from the intensity spectrum. Same as figure 2.28 The upper graphs are plotting the summation of the pixel values of the OPA region vs number of frames. The lower graphs are showing the values in the frequency spectrum. 


\section{Chapter 3}

\section{Reconfigurable Optical Logic Using}

\section{Carrier Depletion and Optical Memory in Microdisk Resonators}

\subsection{Background}

Limited by the von Neumann bottleneck (computer architectures where data operation and instruction fetch cannot occur at the same time) current CPU processors fail to operate at ultrafast speeds for real-time applications such as video analysis, detection of fast-moving objects and LIDAR analysis. Recent trends in transistor size also provides evidence that the Moore's law is reaching its saturation. Photonic systems offering broad bandwidth and high speeds gives us the ambition to shift to a completely different computing paradigm and develop systems such as Photonic Neural Networks (PNN) [7, 28], Photonic Quantum Information [32] and Optical Field Programmable Gate Arrays (FPGA) [19,27]. Optical logic gates with reconfigurable switching modes can be an important piece in a large-scale integrated (LSI) optical logic architecture - an optical equivalent of FPGA. 
Free carrier depletion (FCD) effects have been used widely to perform optical logic operations because of their high speed and low power consumption. FCD based microresonators can be as fast as $128 \mathrm{~Gb} / \mathrm{s}$ with electro-optic bandwidth of $50 \mathrm{GHz}$. [30]. But FCD effects are volatile and not inherently reconfigurable. Phase-Change materials can circumvent this problem with the potential of high-density integration. Electro-thermal switching of PCM materials are relatively slow, of the order of $500 \sim 1000 \mathrm{~Kb} / \mathrm{s}$ [36] and thus we utilize the advantage of both switching mechanisms to propose our reconfigurable logic architecture.

Silicon photonic devices can be actively tuned by widely established thermo-optic (TO) or Electro-Optic (EO) effects which has a refractive index tuning range in the order of 0.01 and 0.0001 respectively. Thus, a large phase shift usually requires long waveguides of around hundreds of micrometers increasing the footprint and complexity of the device. Employing a resonator structure can circumvent this problem although it will at the cost of device bandwidth. Furthermore, these switching mechanisms are volatile and require constant power supply. A non-volatile switch remembers its state without any external power, until a signal is sent, the switch will stay in that state and the process is reconfigurable. Phase Change Materials (PCMs) are good candidates for non-volatile switching since they have large refractive index contrast between their covalent-bonded amorphous state and the resonant-bonded crystalline state over a broad spectral range [35]. Figure 3.1a) optical transmission of a phase change material in amorphous and crystalline states. The large change in refractive index in PCMs can be attributed to the change in cohesive energy of the PCM states. Devices based on optical phase change (O-PCM) materials have been studied because of their large index change properties and reconfigurability [35]. The material can be reversibly transitioned from amorphous to crystalline state and the change of state can introduce a phase change to the flow of light. Figure 3.1b) shows the bandgap energies of the phases of Ge-Sb-Se-Te (GSST)- a phase change material under varying concentration of Se. It can be seen that the cohesive energy of crystalline phase is higher than the amorphous phase making it a more absorptive material. Recent research 
has also shown that, some (O-PCM) materials like Ge2Sb2Te5 (GST) can be tuned quasicontinuously and the intermediate states between amorphous and crystalline state can be achieved at room temperature [37].

O-PCM materials have opened doors for applications in on-chip switching, routing, optical signal processing and optical neuromorphic computing. Due to its sub-ns 'write' speeds when excited by photonic laser pulses, O-PCM materials are envisioned to be used for spike-based neuromorphic processing in the optical domain [6]. These proposed architectures can mimic the behavior of the biological neuron and can be applied to deep neural networks (DNN) and recurrent neural networks (RNN). Their reconfigurability has also been used for controlling the phase front of light. [11]

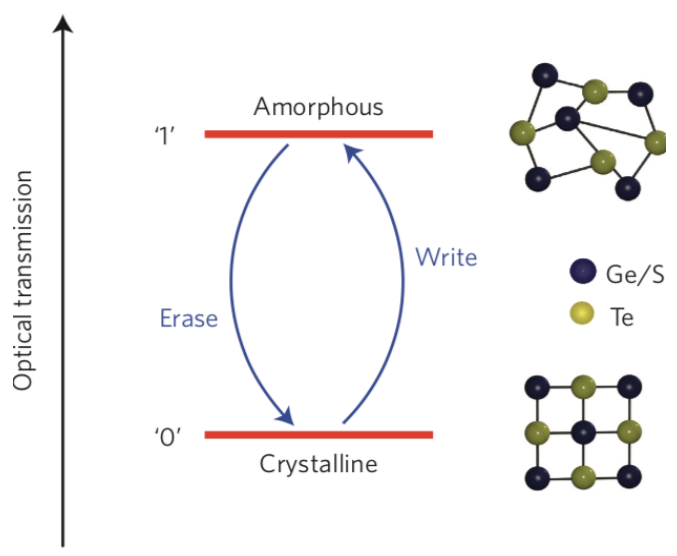

a)

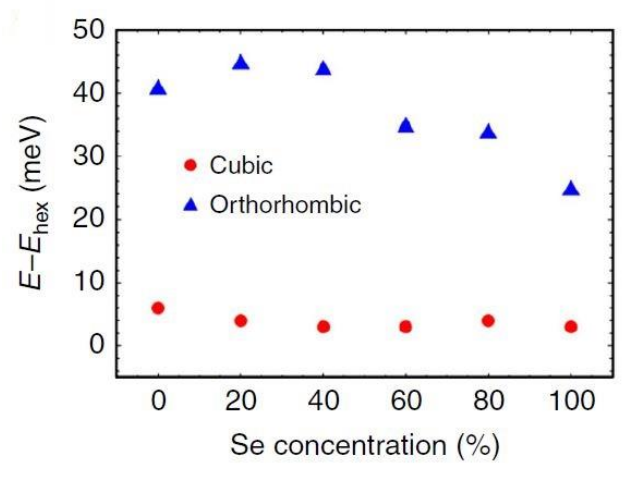

b)

Figure 3.1: a) Optical transmission of a phase change material in amorphous and crystalline states. The amorphous and crystalline states represent write and erase states respectively. As seen from the level scheme, crystalline state is more absorptive than amorphous. The insets show the atomic ordering of the states. Reprinted from [26] b) Cohesive energy plot of cubic and orthorhombic phases of Ge-Sb-Se-Te (GSST)- a phase change material with varying concentration of Se. Reprinted from [36] 
In this thesis, we present a novel optical switch that can be applied to a directed logic architecture that is scalable and reconfigurable. Our proposed switching employs both carrier depletion and phase-change material-based switching on the same device. In a typical reconfigurable switch, two types of resonance tuning are used. One is for the switching controlled by the logic signal and the other is to change the operation mode of the switch. We choose to use carrier depletion to switch the logic signal while the phase-change material to switch the operation modes. Since, we are using a non-volatile switching on the operation modes, they can be reconfigurable without exerting any external power source.

\subsection{GSST cladded Disk Resonator}

In this work, we propose a disk resonator cladded with a relative new class of Optical Phase Change Material (O-PCM), Ge-Sb-Se-Te (GSST) derived from the conventional GeSb-Te (GST) by partially substituting Te with Se. Using either optical or electrical (through Joule heating) excitations, the O-PCM can be reversibly transitioned from low loss amorphous state to high loss and absorptive crystalline state. Optical heating by applying laser pulses provides fast switching speed (switching speed can be of sub-ns timescale) and efficient energy consumption. But this method is not feasible for large-scale integration since the task of aligning laser pulses on each of the O-PCM samples can be slow, diffraction-limited and inaccurate. O-PCMs can also be switched by evanescent coupling of optical pulses from waveguides to O-PCMs but would introduce complex light routing. In this work, we propose using external indium tin oxide (ITO) heaters to switch the PCM states that is a viable option for large-scale integration with switching speed of around 100 microseconds.

We consider a disk resonator with a GSST element cladding as shown in Figure 3.2(a). Wave entering through the INPUT port gets partially coupled to the whispering gallery 
a)

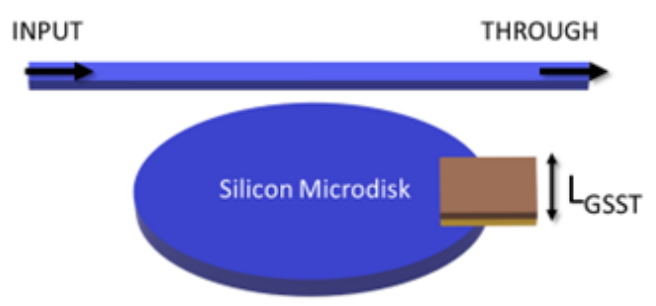

b)

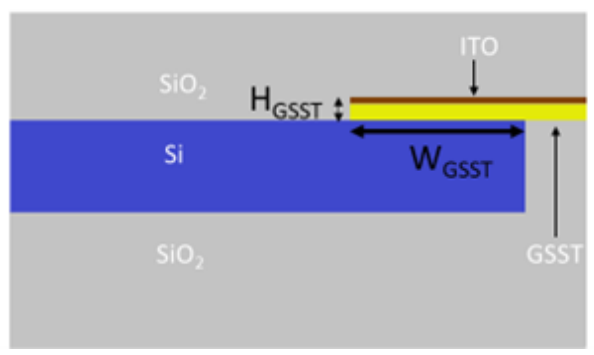

c)

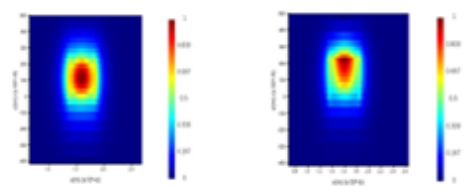

d)

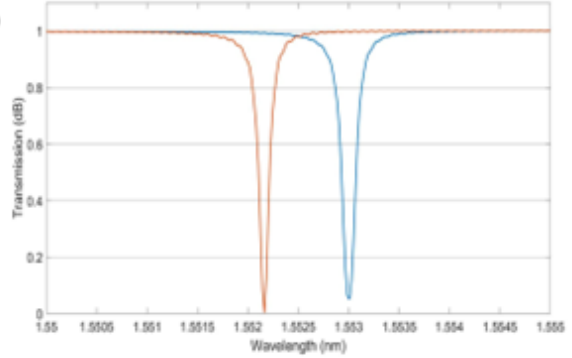

Figure 3.2: (a)Schematic of GSST on Silicon Microdisk with ITO heater on top. (b) Crosssection of the right half of the disk. (c) Evolution of a quasi-TE mode traversing along a Si waveguide with GSST on top. (d) Demonstration of redshift of resonance wavelength with changing the GSST from amorphous to crystalline. The orange and blue plot indicates response in amorphous and crystalline states respectively. Lumerical's FDTD simulation were performed on a Silicon microdisk of $5 \mu \mathrm{m}$ radius and $0.22 \mu \mathrm{m}$ height. Other parameters are: $\mathrm{L}_{\mathrm{GSST}}=1.5 \mu \mathrm{m}, \mathrm{W}_{\mathrm{GSST}}=0.4 \mu \mathrm{m}, \mathrm{H}_{\mathrm{GSST}}=0.025 \mu \mathrm{m}$, height of ITO layer is $0.01 \mu \mathrm{m}$. The amount of redshift we see is around $10 \mathrm{~nm}$.

mode inside the disk. Using simple Fabry-Perot theory we can calculate the resonant modes [12]:

$$
2 \pi R_{\text {disk }} n_{\text {eff }}=m \lambda_{m}
$$

Eq. 3.1 provides the resonant condition (at wavelength $\lambda_{m}$ ) for the ring resonator of radius $R_{d i s k}$ where $n_{e f f}$ is the effective refractive index of the whispering gallery mode. Fig. 3.2b shows the cross-section of the microdisk (schematic not in scale). In Figure 3.2c, we present the modal intensity profiles of a quasi-TE mode through a Si waveguide loaded 
with GSST. When the state of the GSST is in amorphous state, the mode is more spread across $\mathrm{Si}$ and GSST materials with an effective refractive index of $2.80281+0.000111$. On the other hand, when the GSST is in crystalline state the mode is more absorbed towards the GSST material with a higher refractive index of $2.8057+0.00057 \mathrm{i}$. In Figure $1 \mathrm{~d}$ we see the redshift due to changing the state of the GSST material. The dimensions of the microdisk simulations are provided in the figure description.

a)

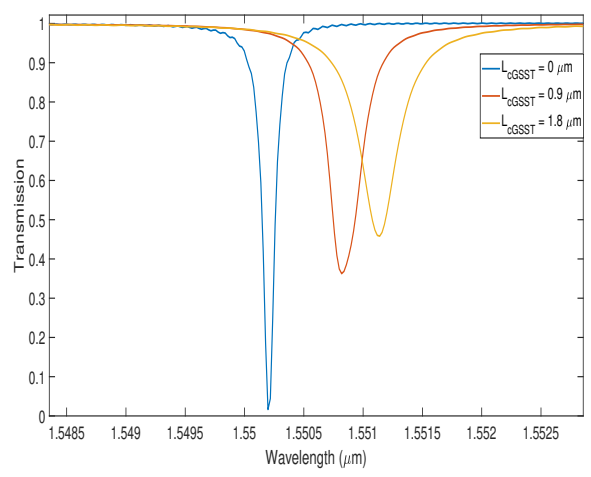

b)

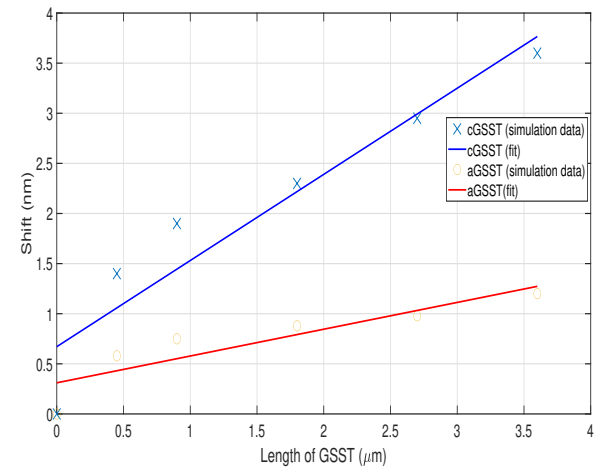

Figure 3.3: (a) Transmission spectra is redshifted as we gradually increase $\mathrm{L}_{\mathrm{cGSST}}$. As the length of the GSST is material is increased, it absorbs more light and shifts the value of effective refractive index causing the spectrum to be redshifted. b) The shift of the resonance peak with gradual increase in the $\mathrm{L}_{\mathrm{cGSST}}$ or $\mathrm{L}_{\mathrm{aGSST}}$. A plot like this helps us identify the length of GSST material required to achieve a certain shift in resonance.

Figure 3.3a shows the evolution of Transmission spectra as the length of GSST strip is increased. It is seen that the shift in resonance peak follows a linear pattern (shown in Fig 3.3b). After fitting the results, we see that the resonant shift per unit GSST length $(\Delta \lambda / L)$ for amorphous and crystalline GSST are $0.2674 \mathrm{~nm} / \mu \mathrm{m}$ and $0.8596 \mathrm{~nm} / \mu \mathrm{m}$ respectively around $1550 \mathrm{~nm}$. Thus, we can express the spectral shift quantitively as [37]:

$$
\frac{\Delta \lambda}{L_{G S S T}} \approx \frac{\Delta n_{e f f} \lambda_{0}}{2 \pi R_{D I S K} n_{e f f, 0}}
$$


Where $n_{e f f, 0}$ is the effective refractive index of the whispering gallery mode (with no GSST overlay) and $\Delta n_{e f f}$ is the real part of the effective index change. Evaluation equation 3.2 using our simulation parameters we find $\Delta / L$ to be $0.30288 \mathrm{~nm} / \mu \mathrm{m}$ and $0.8535 \mathrm{~nm} / \mu \mathrm{m}$ for amorphous and crystalline GSST respectively which is close to our fitted value in Figure 3.3b.

In this section, we have quantitively described the modulation effects and resonance shift as a result of switching the GSST material and increasing it length. This is crucial in deciding the length of GSST we need to align both the carrier depletion resonance and GSST effect resonance at the same wavelength. We will describe the operations in the next sections.

\subsection{Electrothermal Switching of O-PCM with ITO Heater}

In this section we describe the details of 3D time-dependent simulations performed to model the heat transfer of GSST material. Table 3.1 shows the simulation parameters we used in our Multiphysics simulations.

We use a rectangular ITO heater of dimensions $3 \times 3 \mu \mathrm{m}^{2}$ and height $10 \mathrm{~nm}$ to heat the GSST layer. Figure 3.4a shows the heat map produced by Joule heating the GSST material. Figure $3.4 \mathrm{~b}$ shows the switching dynamics of amorphous to crystalline and figure $3.4 \mathrm{c}$ shows the vice versa. Around $900 \mathrm{~K}$ of temperature is required to melt the GSST into amorphous state, whereas for inducing re-crystallization, the GSST needs to be heated above the crystallization temperature $(\sim 523 \mathrm{~K})$ but below the melting point, for a critical amount of time, thus requires multiple pulses [23].

The crystallization to amorphization process takes approximately $1.1 \mu \mathrm{J}$ per switch cycle with cycle length of $1.5 \mu \mathrm{s}$. The reverse process takes almost $4.1 \mathrm{~mJ}$ per switch cycle with duration of approximately $70 \mu \mathrm{s}$. 
Table 3.1: Parameters used for thermal simulations in COMOSOL Multiphysics

\begin{tabular}{|c|l|l|l|}
\hline Material & $\begin{array}{l}\text { Thermal } \\
\text { Conduc- } \\
\text { tivity } \\
(\mathrm{W} / \mathrm{m} . \mathrm{K})\end{array}$ & $\begin{array}{l}\text { Heat } \\
\text { Capacity } \\
\left(\mathrm{MJ} / \mathrm{m}^{3} / \mathrm{K}\right)\end{array}$ & $\begin{array}{l}\text { Electrical } \\
\text { Con- } \\
\text { ductiv- } \\
\text { ity }(\mathrm{S} / \mathrm{m})\end{array}$ \\
\hline ITO Heater $(10 \mathrm{~nm})$ & $1.16[33]$ & $2.6[33]$ & $\begin{array}{l}8.6 \times 10^{3} \\
{[18]}\end{array}$ \\
\hline GSST (amor) & $0.18[23]$ & $1.5[23]$ & $0.2[36]$ \\
\hline GSST (crys) & $0.43[23]$ & $1.9[23]$ & $5000[36]$ \\
\hline
\end{tabular}

a)
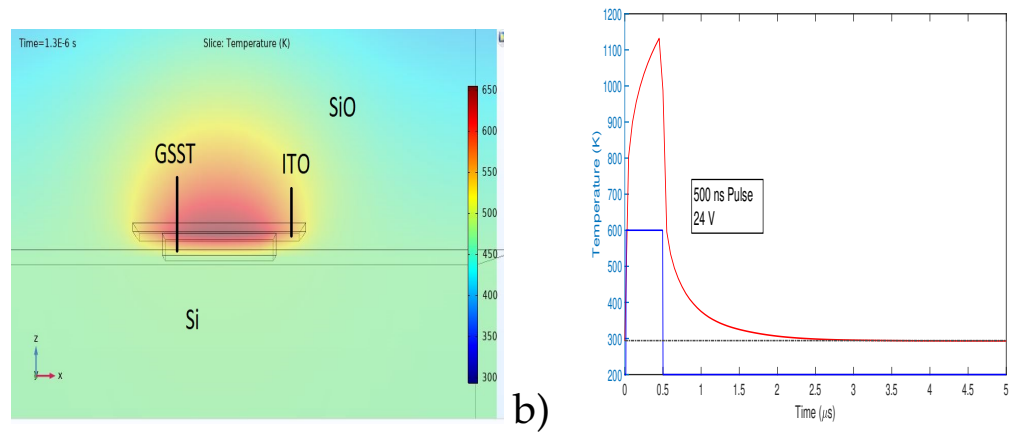

c)

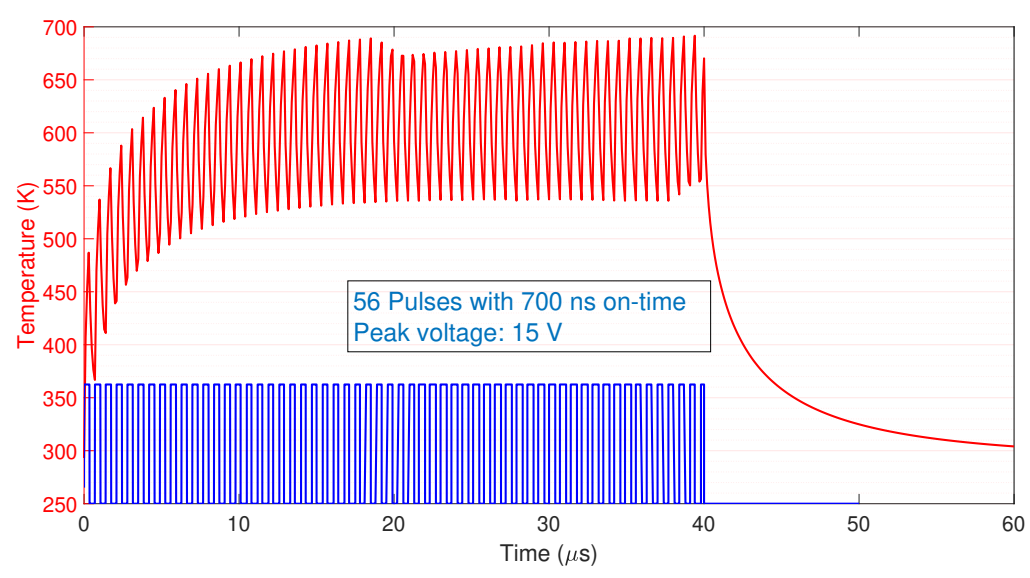

Figure 3.4: Electro-thermal switching from amorphous to crystalline through Joule heating and vice versa. a) Heat map produced by Joule heating. b) Temperature average in the GSST layer as a function of time for rectangular pulse heater. For amorphization, the temperature is kept above $900 \mathrm{~K}$ (amorphization temperature) by applying a single rectangular pulse of $24 \mathrm{~V}$. c) For crystallization, the average temperature is kept above $523 \mathrm{~K}$ (crystallization temperature) but below $900 \mathrm{~K}$ for more than $20 \mu \mathrm{s}$. 


\subsection{Carrier Depletion in Silicon Microdisks}

In this section we present microdisk modulators with a double-shift vertical PN junction. The vertical PN junction is more desired than lateral junctions because the depletion region developed in vertical junctions is more distributed along the whispering gallery mode and thus reduces the voltage requirement. Figure 3.5a presents a schematic of PN junction controlled microdisk modulator. We perform Finite Element Method (FEM) simulations using Lumerical's DEVICE software to calculate the charge density profile that would eventually be imported into MODE model to simulate the refractive index change due to applied bias. For our purpose of implementing logic operations, we choose a $\mathrm{Si}$ disk of radius $5 \mu \mathrm{m}$ buried in oxide. We believe this device size is optimum in having both carrier depletion and PCM switching in the same microdisk. The bus waveguides are $0.4 \mu \mathrm{m}$ wide and the spacing between the disk and waveguides are $0.16 \mu \mathrm{m}$. Figure $3.5 \mathrm{~b}$ shows a cross section of the vertical junction with defined concentration regions. We use n-doped Silicon of concentration of $4 \times 10^{18} \mathrm{~cm}^{-3}$ and p-doped Silicon of concentration $2 \times 10^{18} \mathrm{~cm}^{-3}$ at the edge of the Silicon disk. Underneath the anode we have highly doped $\mathrm{p}+$ Silicon of concentration $1 \times 10^{20} \mathrm{~cm}^{-3}$ and below the cathodes we have highly doped $\mathrm{n}+$ Silicon of concentration $2 \times 10^{20} \mathrm{~cm}^{-3}$. Figure 3.6a shows the depletion region for the vertical junction with an applied bias of $3.4 \mathrm{~V}$. The transmission response of the disks are simulated using varFDTD approach in Lumerical. The applied bias shifts the resonance peak by $72.3 \mathrm{pm} / \mathrm{V}$ ( $246 \mathrm{pm}$ for $3.4 \mathrm{~V}$ applied bias). The disk has a $\mathrm{Q}$ factor of approximately 4020 with FWHM $=390.74 \mathrm{pm}$. In figure $3.6 \mathrm{~b}$ the transmission response of the disc resonator in the drop port is presented with applied biases of $0 \mathrm{~V}$ and $3.4 \mathrm{~V}$. The total switching delay is defined as $\tau_{\text {switch }}=\tau_{R C}+\tau_{\text {photon, }}$, where $\tau_{R C}$ is the RC constant of the metallic contacts and $\tau_{\text {photon }}$ is the photon lifetime calculated to be $3.35 \mathrm{ps}$. 

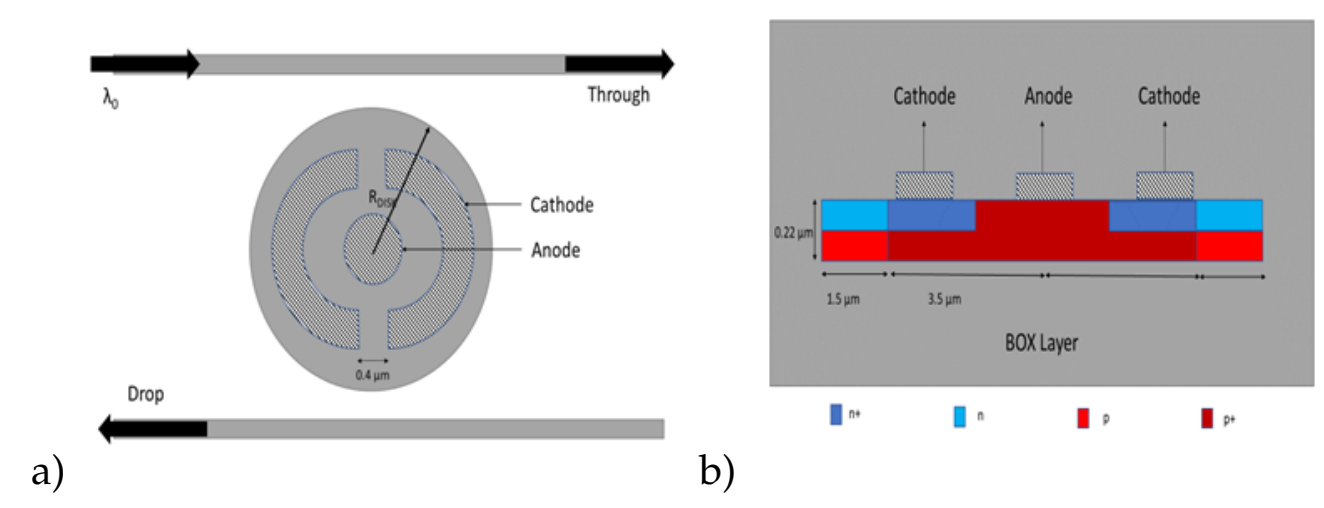

b)

Figure 3.5: (a) Schematic of the double-shift vertical PN junction. The graded region indicate the metal contacts to switch the microdisk. (b) Cross section of the microdisk buried in oxide layer with $\mathrm{p}, \mathrm{n}, \mathrm{p}+$ and $\mathrm{n}+$ regions defined.

\subsection{Switching and Logic Architecture}

For our switching we use a disc resonator with vertical PN junction as shown in figure 3.7a. Figure $3.7 \mathrm{~b}$ shows the transmission response at the drop port of the device under different switching states of the PCM and PN-junction.

Figure $3.2 \mathrm{~d}$ shows us that by changing the state of the PCM we can obtain a red shift in the resonance peak. Thus, we can find a specific amount of bias in the PN-junction that will produce an amount of blue shift in the spectrum. We can use this to tune the disk to be at block/pass, pass/block or block/block mode.

In a reconfigurable switch, two types of resonance tuning are used. One is used for logic operation while the other for sending a reconfigurable signal. By blue shifting the resonance peak we can use the microdisk to any specific logic operation such as NAND gate. The state of the PCM material is changed to change the operating mode of the disk and thus we can switch between logic operations such NAND to OR and vice versa. Figure 3.7 shows a way to realize two logic operations at a single wavelength. When the disk is under resonance, the disk blocks the path of the light for the through port and most light passes along the through port when the disk is off-resonance. Thus, we can label a photodetector along the through port with specific logic operations and switch the 


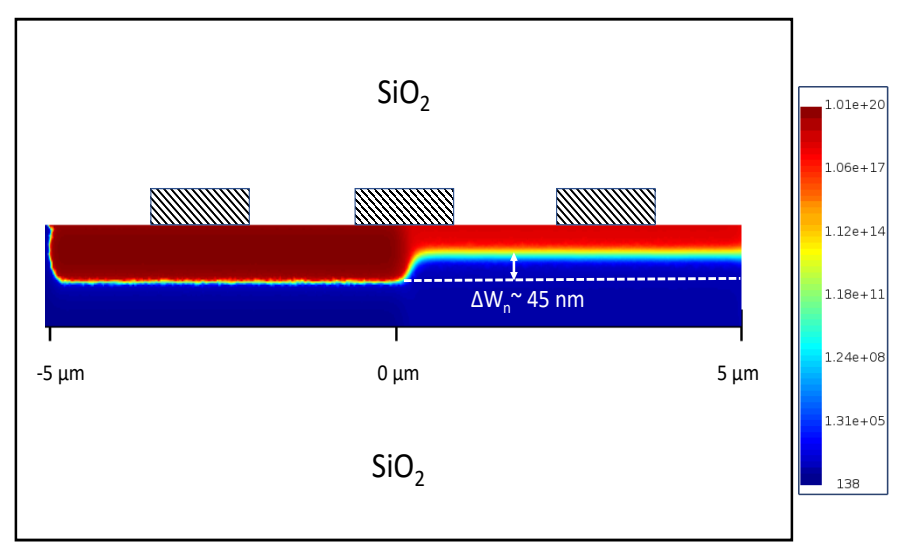

a)

b)

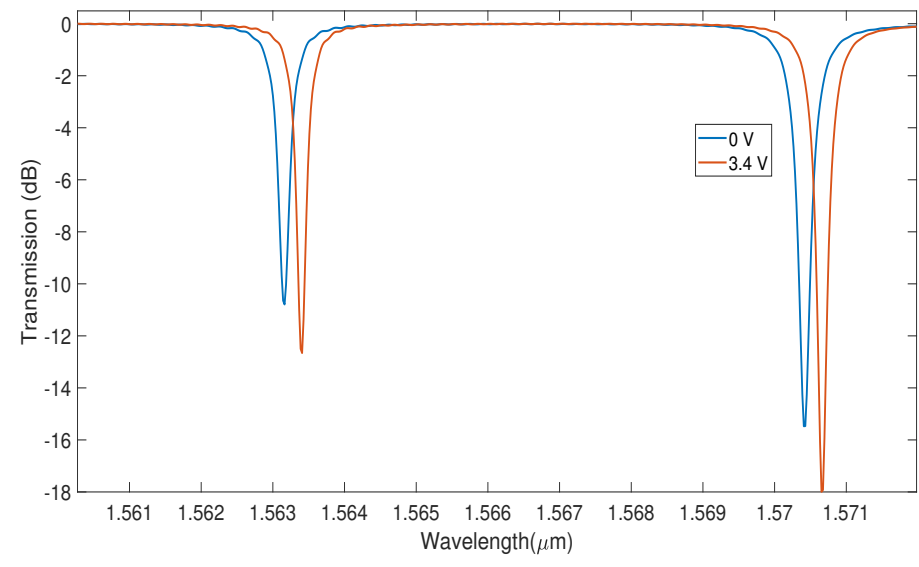

Figure 3.6: a) Carrier profile of free electrons of the vertical junction simulated through FEM simulation when $3.4 \mathrm{~V}$ of reverse bias is applied on the right of the microdisk. The right side of the disk shows a $45 \mathrm{~nm}$ of larger depletion width. b) Transmission response at the drop port of the microdisk with applied biases of $0 \mathrm{~V}$ and $3.4 \mathrm{~V}$.

pass/block states to perform logic operations. This labelling will also depend on which state we are keeping the PCM material. Table 3.2 shows the states of the PCM and PNjunction bias applied to demonstrate logic operations. 


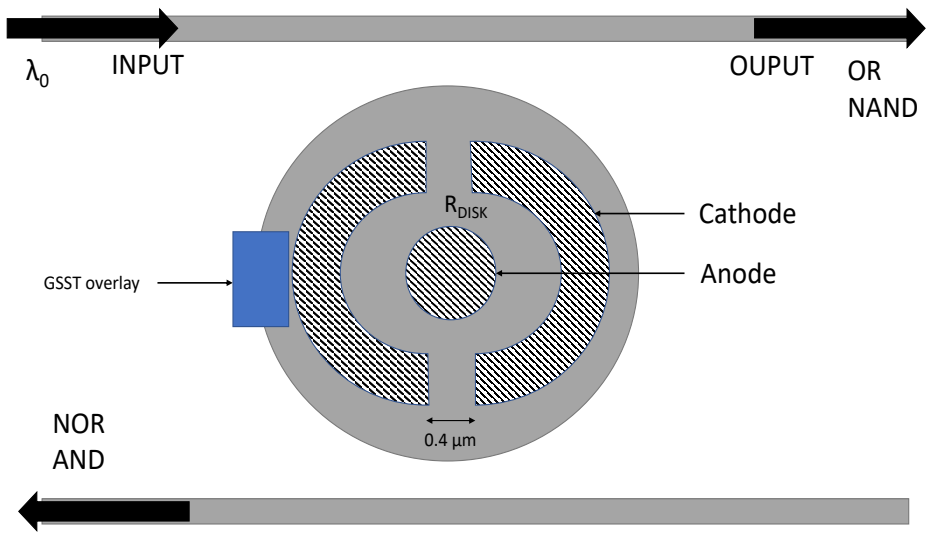

a)

b)

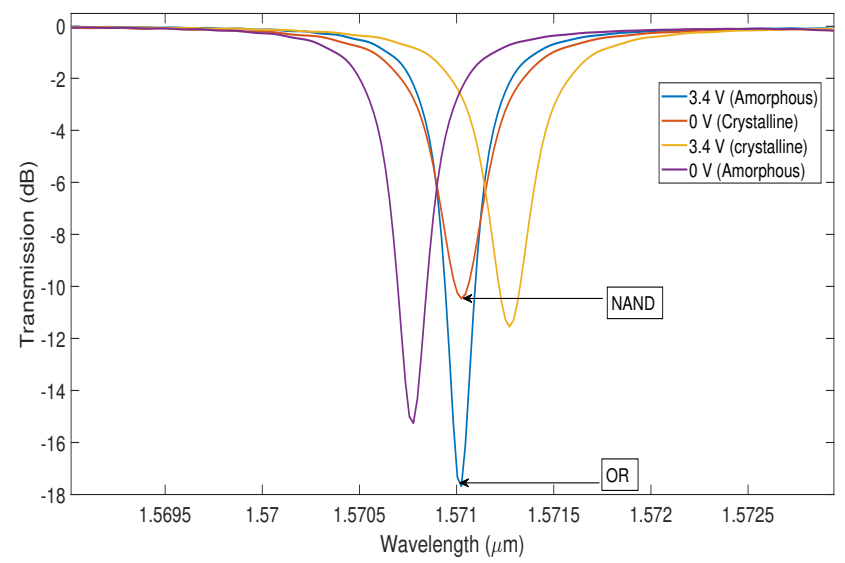

Figure 3.7: a) Schematic of the logic cell with 2x logic operations. The PCM strip has a length of $400 \mathrm{~nm}$, a width of $200 \mathrm{~nm}$ and $25 \mathrm{~nm}$ of height. b) Transmission response under different states of PCM and PN-junction. Two logic operations can be carried out at wavelength $1.571 \mu \mathrm{m}$

Table 3.2: Blueprint for 2x logic operation

\begin{tabular}{|c|l|l|}
\hline Logic Operation & $\begin{array}{l}\text { PN Junc- } \\
\text { tion } \\
\text { reverse } \\
\text { Bias }\end{array}$ & PCM State \\
\hline NAND & $0 \mathrm{~V}$ & Crystalline \\
\hline OR & $3.4 \mathrm{~V}$ & Amorphous \\
\hline
\end{tabular}




\subsection{Higher Order Logic}

The optical logic cell that we described in previous section can serve as the elementary unit of a higher order logic. One common approach to designing higher order logic is to cascade the logic cells with OE conversions in between. But OE conversions add inherent delays and increase overall footprint. We propose directed logic architectures where a number of microdisks are cascaded and operated based on which disks are on or off resonance. The introduction of optical memory allows fewer disks in our architectures and thus improves the overall latency. We propose an N-bit comparator and N-bit adder network in this work.

\subsubsection{N-x Logic Operation}

Figure 3.8a shows the schematic of a logic cell with $3 x$ logic operations in a single disk. We can achieve $n-x$ operations of logic in a single microdisk by having more PCM strips on a single disk considering the fact that more PCM strips will increase the overall insertion loss of the disk and will require higher bandwidth of operation. Figure $3.8 \mathrm{~b}$ shows the transmission response of a $5 \mu \mathrm{m}$ microdisk with two PCM strips for reconfigurable operations and a U-type pn-junction switching for logic operation. Table 4.1 shows the states of the PCM and PN-junction bias applied to demonstrate logic operations. A Utype $\mathrm{PN}$-junction [5] is used to demonstrate $3 \mathrm{x}$-operation because of lower insertion loss. Microring modulators with U-shaped PN-junctions have been demonstrated in [?] with improved modulation efficiency and lower loss. Thus, with better modulation efficiency and lower loss resonator structures we have the ability to demonstrate n-x logic operations in a single disk.

\subsubsection{N-bit Comparator}

Figure 3.9 shows a 4-bit optical comparator. A comparator determines if two numbers are equal or one is greater than the other. The reconfigurable states $M_{0}$ and $M_{1}$ determine the 


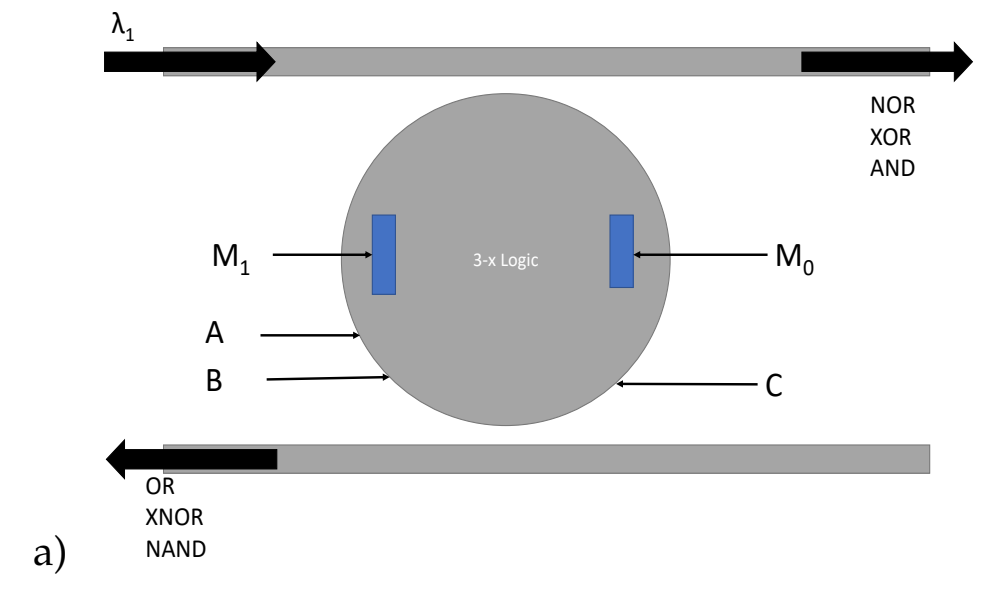

b)

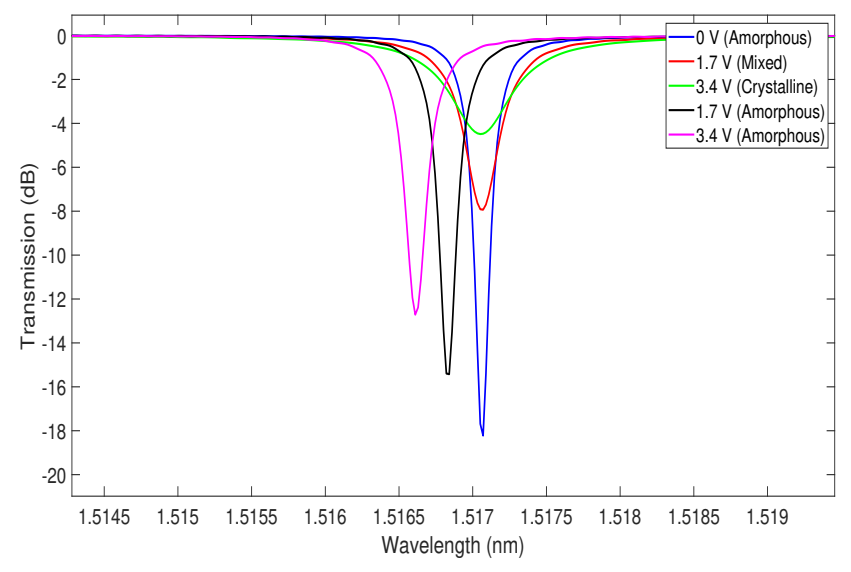

Figure 3.8: (a) Schematic of a logic cell with 3x operations in a single disk and at a fixed wavelength. This cell can be scaled up to nx operations. (b) Transmission response of a logic cell of similar functionality. The blue, red and green curves represent amorphous state with $0 \mathrm{~V}$ bias, mixed state with $1.7 \mathrm{~V}$ bias and crystalline state with $3.4 \mathrm{~V}$ bias respectively. The black and purple curves represent amorphous states with $1.7 \mathrm{~V}$ and $3.4 \mathrm{~V}$ applied Bias. We can see that by changing the state of the PCM we can shift the resonance peak and align them with the initial state. This can be utilized for our $3 x$ operation logic cell. 
Table 3.3: Blueprint for $3 x$ logic operation

\begin{tabular}{|c|l|l|}
\hline Logic Operation & $\begin{array}{l}\text { PN Junc- } \\
\text { tion } \\
\text { reverse } \\
\text { Bias }\end{array}$ & PCM State \\
\hline NAND & $0 \mathrm{~V}$ & Amorphous \\
\hline XNOR & 1.7 & $\begin{array}{l}\text { Mixed } \\
\text { (Amorphous- } \\
\text { Crystalline) }\end{array}$ \\
\hline OR & $3.4 \mathrm{~V}$ & Crystalline \\
\hline
\end{tabular}

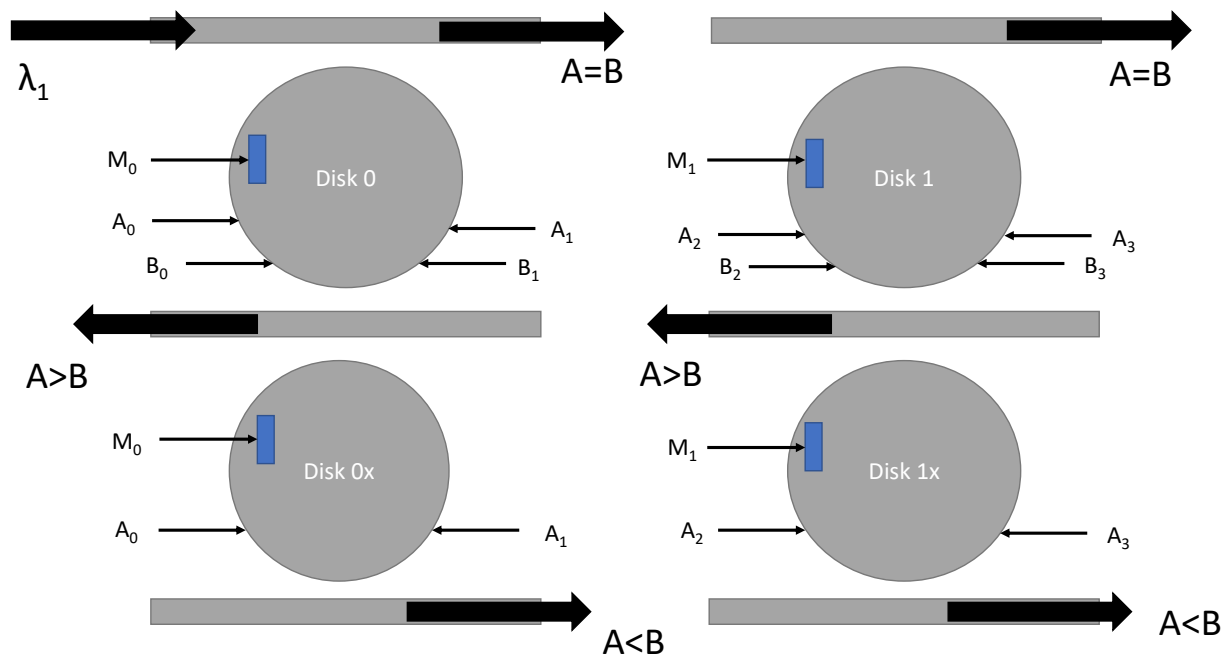

Figure 3.9: Schematic of a 4-bit comparator. The through port determines if two 4-bit numbers $(\mathrm{A}$ and $\mathrm{B})$ are equal. The drop ports determine if $\mathrm{A}$ is greater than $\mathrm{B}$ or not.

operating mode of the disks. When $M_{0}$ is on, disk 0 performs the $A_{0} \oplus B_{0}$ operation for the through port and so on. If $A_{0} \neq B_{0}$, disk 0 works on resonance and turns on $A_{0}>B_{0}$ if disk $0 \mathrm{x}$ is off resonance. If both disks 0 and $0 \mathrm{x}$ are on resonance, then $A_{0}<B_{0}$. The presented architecture requires $\mathrm{N} / 2$ number of disks for N-bit comparator operation. In comparison to the work presented in [17] that required N-disks for N-bit comparator, this architecture requires $\mathrm{N} / 2$ disks with $\mathrm{N} / 2$ switching of the PCM state which is slower than FCD switches as presented in previous sections. Thus, with a trade-off of speed and 
operational complexity we can achieve a more compact and power-efficient architecture with this design.

\subsubsection{Optical Adder}

Figure 3.10 shows the possible schematic of a 2-bit optical adder with our logic cell. This design utilizes the reconfigurability of the PCM material and requires fewer disks than presented in [17] with a trade-off of overall operational speed and additional O/E conversion. The outputs of the full adder can be defined as:

$$
\begin{array}{r}
S_{1}=A_{1} \oplus B_{1} \oplus C_{\text {in }} \\
S_{2}=C_{\text {out }}=A_{1} \cdot B_{1}+C_{\text {in }}\left(A_{1} \oplus B_{1}\right)
\end{array}
$$

Based on the state of $M_{0}$ the cell performs the adder operation with a requirement of 3N-3 disks for an N-bit operation. Disk 1 produces $S_{0}$ and $C_{i n}$ as functions of $A_{0} \oplus B_{0}$ and $A_{0} B_{0}$ respectively. Disk 2 and 3 either produces $S_{1}$ or $S_{2}$ based on the state of $M_{0}$. For the operation of $S_{1}$, Disk 2 performs the operation $A_{1} \oplus B_{1}$ and Disk 2 finally performs $A_{1} \oplus B_{1} \oplus C_{i n}$ when $M_{0}=0$. For the operation of $S_{2}$, disk 2 performs the $A_{1} B_{1}$ operation and disk 3 performs $C_{i n}\left(A_{1} \oplus B_{1}\right)$ when $M_{0}=1$.

\subsection{Summary}

In this chapter we described the dynamics of O-PCM switching on a disk resonator. We found a quantitative way to find the amount of resonance shift due to the introduction of PCM on a disk resonator. This chapter also presented the simulation results of pnmodulated disks and proposed logic cells with both PCM and pn-modulated microdisks. Finally, higher order logic architectures were proposed that required fewer resonators than previous works. 

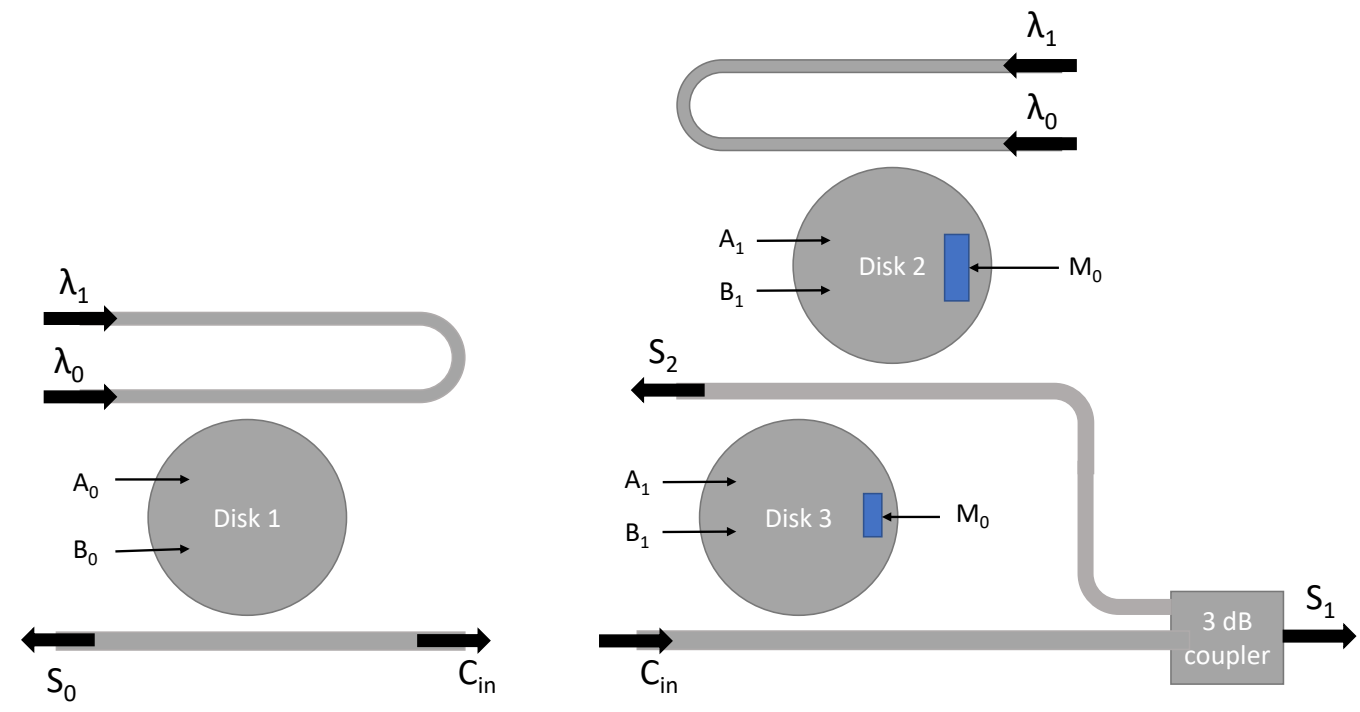

Figure 3.10: Schematic of a 2-bit optical adder. For $\mathrm{N}$ bit operations this cell would require 3N-3 disks. 


\section{Chapter 4}

\section{Non-volatile Switching for Wavelength Selective Spatial Routing}

\subsection{Non-volatile Switching}

Non-volatile switching means the switch remembers its state without any external power. Until a signal is sent, the switch will stay in that state and the process is reconfigurable. Phase Change Materials (PCMs) are good candidates for non-volatile switching since they have large refractive index contrast between their covalent-bonded amorphous state and the resonant-bonded crystalline state over a broad spectral range [35]. Waveguides loaded with PCMs are the basic components to construct non-volatile switching devices. It is possible to realize non-blocking architectures with arbitrary network complexity. These low-loss, nonvolatile switching technology can be found in various applications such as Photonic Neural Networks, Optical Communications, Optical Signal Processing and Field-Programmable Reconfigurable Photonics. [24] 


\subsection{Wavelength-Selective Spatial Routing}

From network architecture perspective, photonic interconnection networks can be classified into wavelength routing, spatial routing and time division multiplexing as shown in Figure 4.1. Wavelength routing is usually done with passing microring or microdisk switches where the optical signals are routed according to their wavelength divisions. This technique provides orders of magnitude higher bandwidth density and thus can accommodate spectrally-parallel streams of data in a single waveguide. However, this architecture is limited by the need of large numbers of light sources and switches operating at specific wavelengths. [8]

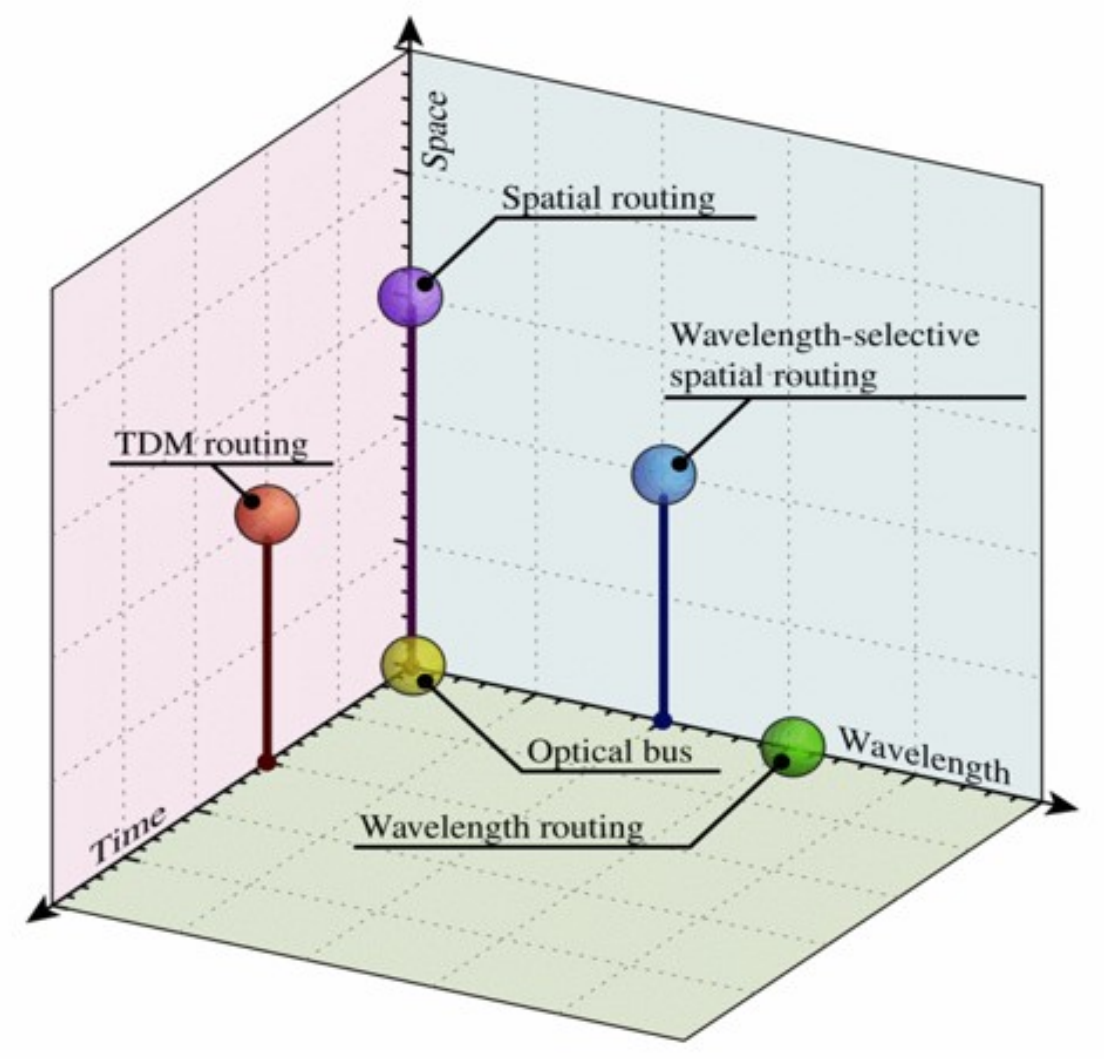

Figure 4.1: Routing techniques available on photonic interconnection networks, reprinted [8] 
The time division multiplexed (TDM) routing is based on temporal positioning of the optical signals. TDM routing establishes node-to-node optical lightpaths by using resonator structures throughout the network, which are tuned to transmit under a continuous series of time frames called time slot. Using a global clock, the resonators are used to guide an optical signal by tracking the time slot of each switch.

In the space domain, it is possible to route an entire spectrum of channels along an optical path [10]. The switching action can be triggered by actively controlling ring resonators or Mach-Zehnder Interferometers (MZIs). It is also possible to achieve wavelengthselective spatial routing where the network architecture provides greater path diversity and energy efficient performance.

\subsection{Photonic Interconnection Network Architectures}

In this section, we present some of the interconnection network architectures proposed with photonic switches. An example of such network is TorusNX topology [9] - that mimics the connectivity of a folded torus for transmitting wavelength-parallel messages. Figure 4.2 shows the schematic of a $4 \times 4$ TorusNX topology. Through simulations, the worst-case insertion loss of a $16 \times 16$ insertion loss was found to be $19.93 \mathrm{~dB}$ [2] with an optical power budget of $40 \mathrm{~dB}$.

Figure 4.3 shows the schematic of an $N \times N \times M$ switch that contains $N \times N$ crossbar switch with $M$ wavelengths per input port [22]. Their approach requires only $2 N^{2}$ rings for an an $N \times N \times M$ switch as compared to other approaches that required $M\left(N \cdot \log _{2}(N)-\right.$ $N / 2$ rings. Their switch had a measured off-resonance and on-resonance loss of $0.17 \mathrm{~dB}$ and $1.9 \mathrm{~dB}$ respectively. 


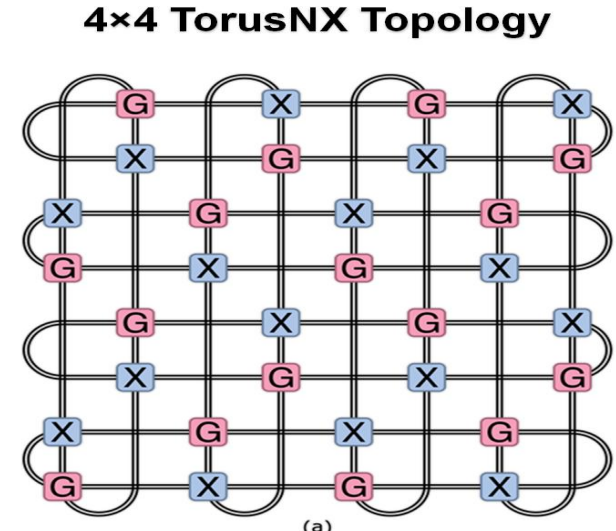

(a)
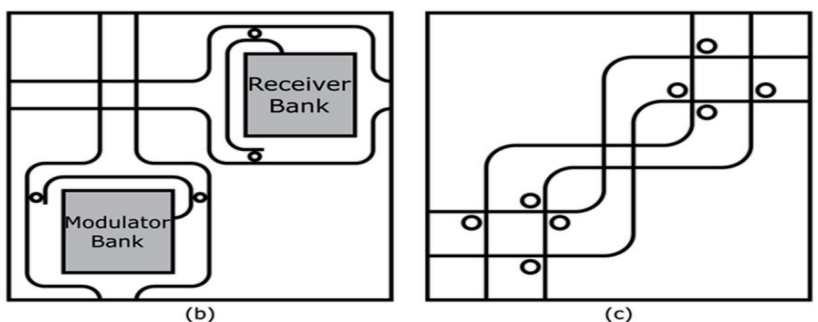

Figure 4.2: (a) A $4 \times 4$ TorusNX topology reprinted from [2]. The $G$ labels represent the gateways and $X$ represent $4 \times 4$ non-blocking switches. (b) Layout of the gateway. (c) Layout of the $4 \times 4$ non-blocking switch.

\section{4 $2 \times 2$ Photonic Switch with Coupled Ring Resonators}

In [35] the authors present a nonvolatile on-chip switch in the telecommunication C-band with record low loss and crosstalk. Their work was based on a new class of O-PCM material $\mathrm{Ge}_{2} \mathrm{Sb}_{2} \mathrm{Te}_{5}$ (GST) which exhibits significantly lower optical loss compared to conventional O-PCM materials such as $\mathrm{Ge}_{2} \mathrm{Sb}_{4} \mathrm{Se}_{2} \mathrm{Te}_{5}$ (GSST). The new O-PCM, Ge-Sb-Se-Te or GSST, is derived from the conventional GST alloy by partially substituting Te with Se. Figure 4.4 compares the optical constants of GSST with GST. Finally, they propose a 2x2 switch with directional coupling mechanism, as shown in Fig 4.5:

Footprint is a disadvantage with directional coupler switches. Although, they were able to achieve very low optical attenuation, it was almost $40 \mu \mathrm{m}$ long with $20 \mathrm{~nm}$ bandwidth. So, we propose a switching device with double ring-resonator structure shown 


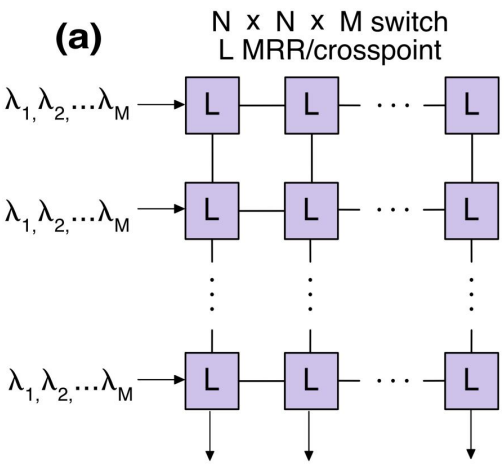

(b) Switch unit cell

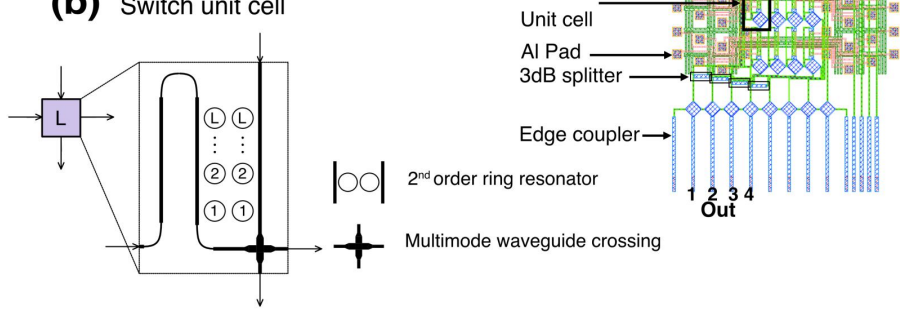

(d)

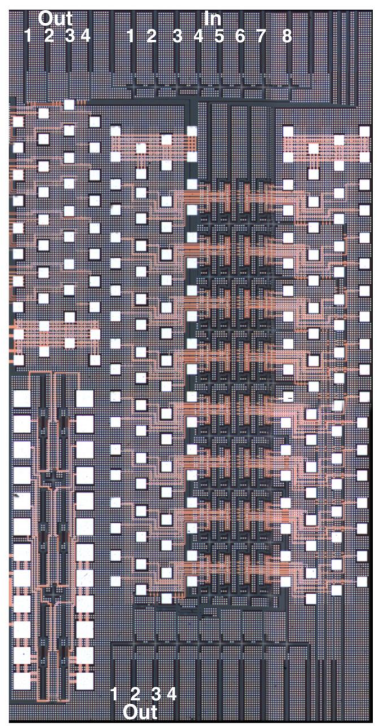

Figure 4.3: (a) Schematic of an $N \times N \times M$ switch with L Micro Ring Resonators per crosspoint (b) Unit cell of the switch (c) Layout of an $8 \times 4$ switch (d) Die Shot of the switch with I/O marked. Reprinted from [22]

in Fig 4.6 with much compact structure. The phase change induced by the GST material is non-volatile and can be freely transitioned from amorphous (low loss) to crystalline (high loss and absorptive). Fig. 4.6 illustrates the cross-state (a) and bar-state (b) of the switch; while (c) shows an example of a wavelength-selective crossbar switch. In Figure 4.7, we present the modal intensity profiles of a Si waveguide loaded with a GST strip in the amorphous and crystalline states. We also show 3D FDTD simulations of the electric field distribution of the $2 \times 2$ switch.

Using the double-ring switches, we present in Fig. 4.9 , a $2^{m} \times 2^{m}(m=2)$ Benes network switch, a simple nonblocking multistage circuit-switching network. 3D FDTD simulations were performed for the proposed switch. Based on the fabrication-feasible dimensions reported in literature, we consider the following geometrical parameters in our simulations: ring resonators of radius $1.5 \mu \mathrm{m}$, waveguide dimensions of $0.45 \times 0.22 \mu \mathrm{m}^{2}$, bus waveguide dimensions of $0.35 \times 0.22 \mu \mathrm{m}^{2}$, bus-to-ring gaps of $50 \mathrm{~nm}$, ring-to-ring gap of $75 \mathrm{~nm}$, GST overcladding of height $10 \mathrm{~nm}$ and length of $170 \mathrm{~nm}$. Our proposed ring 
(a)
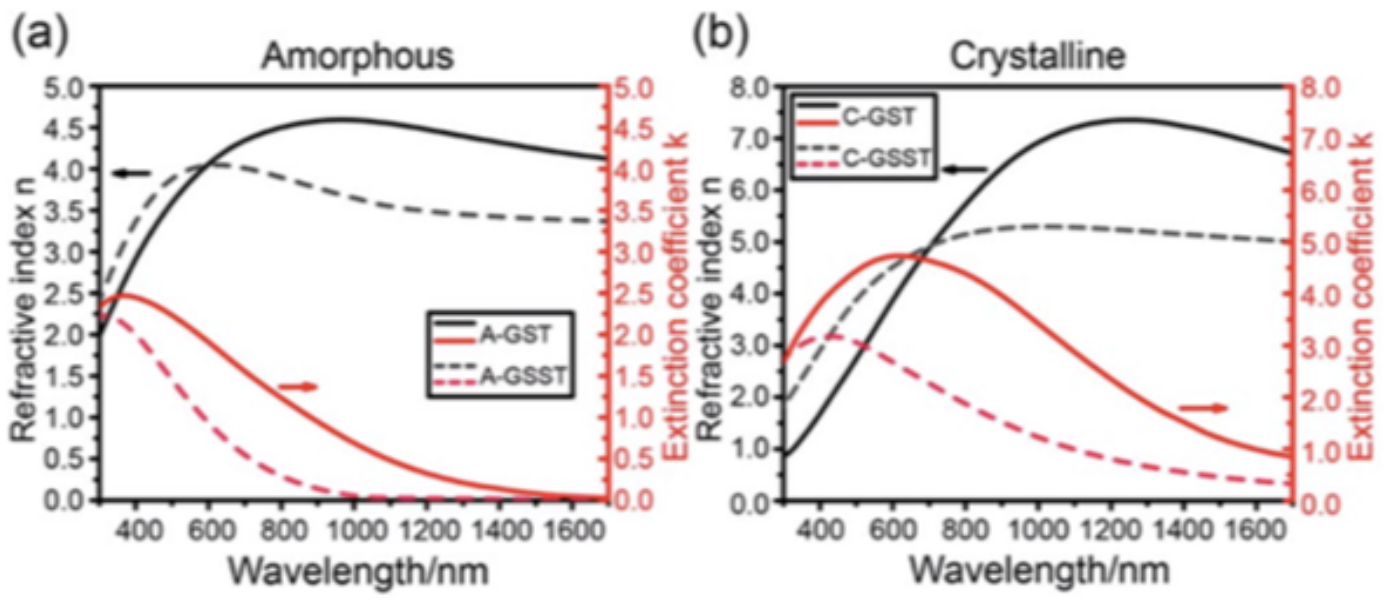

Figure 4.4: Optical constants of (a) amorphous and (b) crystalline phases of GST (solid lines) and GSST (dashed lines) from the visible range to near-infrared. The black lines represent the refractive index $n$, and the red curves are the extinction coefficient $k$. Reprinted [35]

(a)

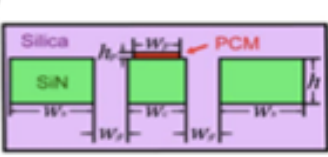

(b)

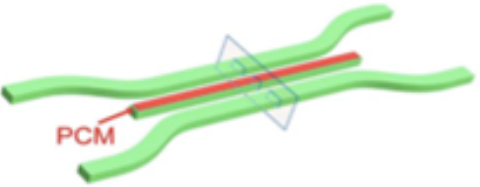

(c)
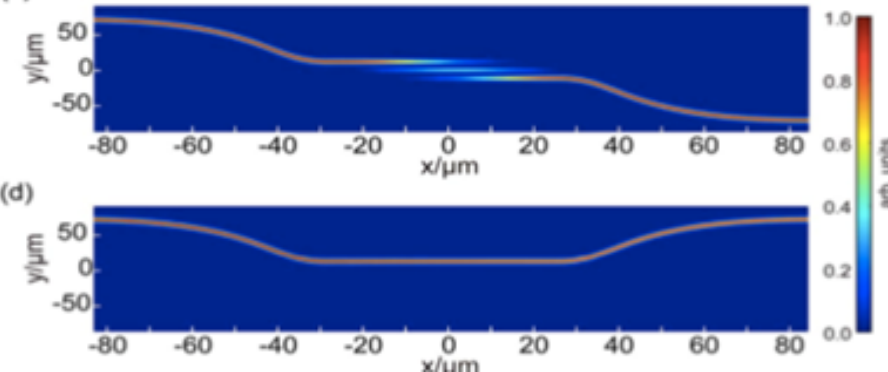

Figure 4.5: Proposed 2x2 switch in [35]: (a) cross section and (b) perspective view of the switch. Here $w_{c}=512 \mathrm{~nm}, w_{s}=730 \mathrm{~nm}, w_{p}=400 \mathrm{~nm}, w_{g}=562 \mathrm{~nm}, \mathrm{~h}=450 \mathrm{~nm}$, and $h_{p}=$ $60 \mathrm{~nm}$; (c) and (d) FDTD simulated field intensity distribution in the device at the (c) amorphous (cross) and (d) crystalline (bar) states.

configuration fits in a footprint of $8 \times 8 \mu \mathrm{m}^{2}$, significantly more compact than the $40 \mu \mathrm{m}$ long directional couplers presented in a similar Benes network switch [35]. For the network switch in Fig. 4.9, we achieve a minimum bar-state and cross-state insertion loss (IL) of $0.63 \mathrm{~dB}$ and $2.81 \mathrm{~dB}$, with a $3-\mathrm{dB}$ bandwidth of $7.2 \mathrm{~nm}$. Furthermore, we extended 


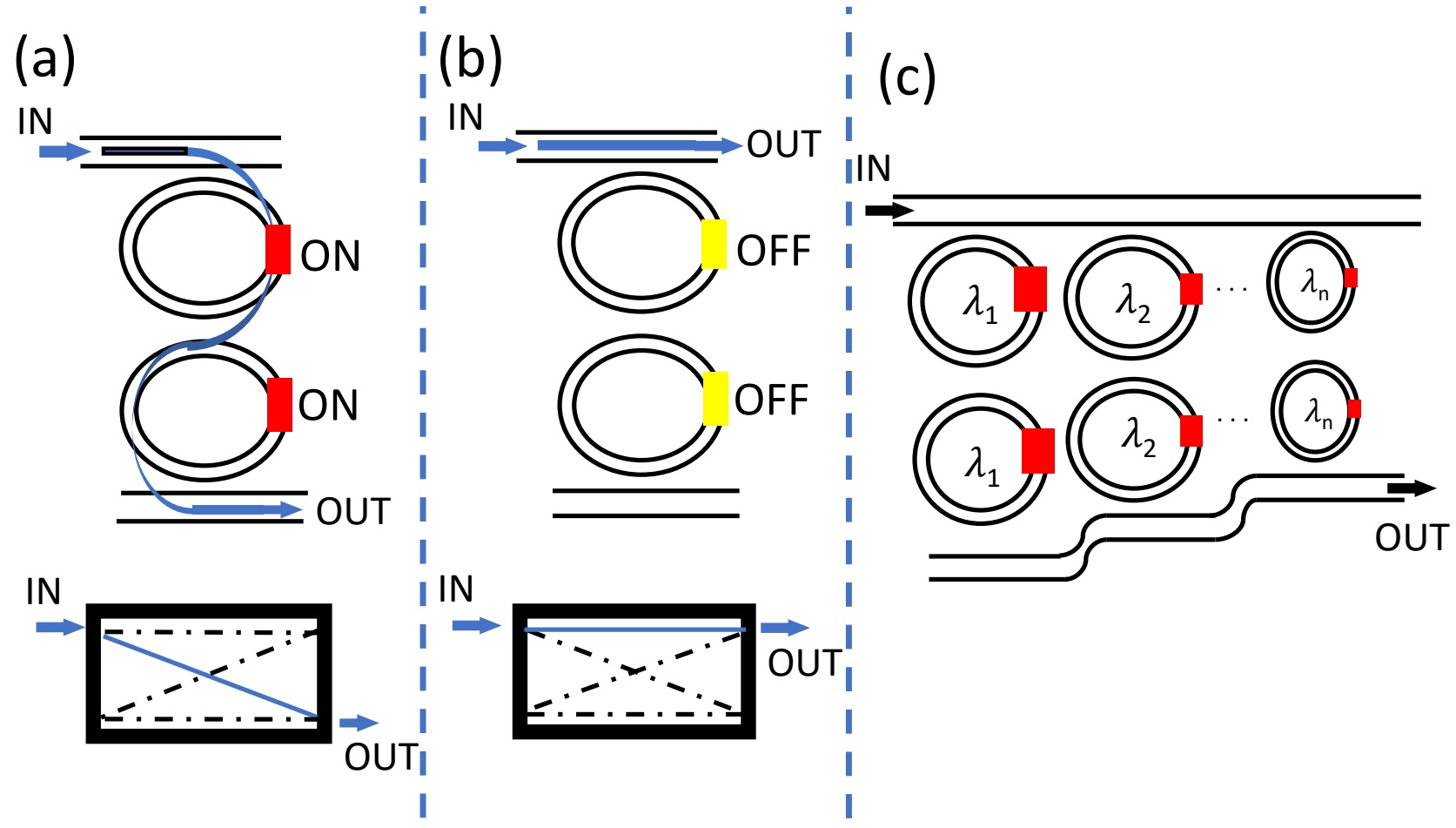

Figure 4.6: Proposed $2 \times 2$ ring resonator-based switch. Red and yellow indicate amorphous and crystalline states of the GST, respectively. (a) and (b): different states of the switch; (c) wavelength-selective crossbar switch.

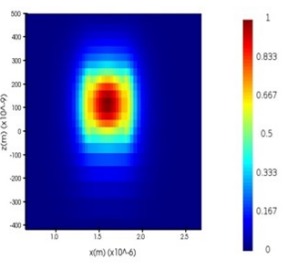

(a)

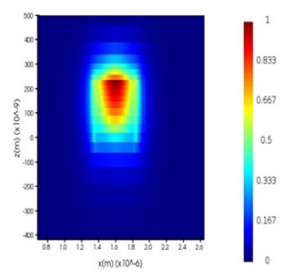

(b)

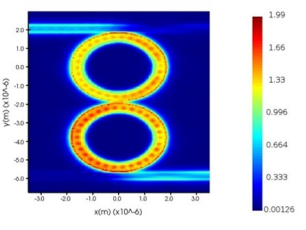

(c)

Figure 4.7: Modal intensity profiles of a Si-waveguide cladded with a GST strip in the (a) amorphous and (b) crystalline states. (c) FDTD simulated field intensity distribution in the amorphous state.

our analysis to GSST overcladding and achieved a bar-state and cross-state insertion loss (IL) of $0.7 \mathrm{~dB}$ and bar-state IL of $0.13 \mathrm{~dB}$ with a 3-dB bandwidth of $1.7 \mathrm{~nm}$. Finally, we use this $2 \times 2$ switch as a building block to assemble a Benes network, a nonblocking switching fabric architecture that can be scaled to realize arbitrary network complexity levels. 


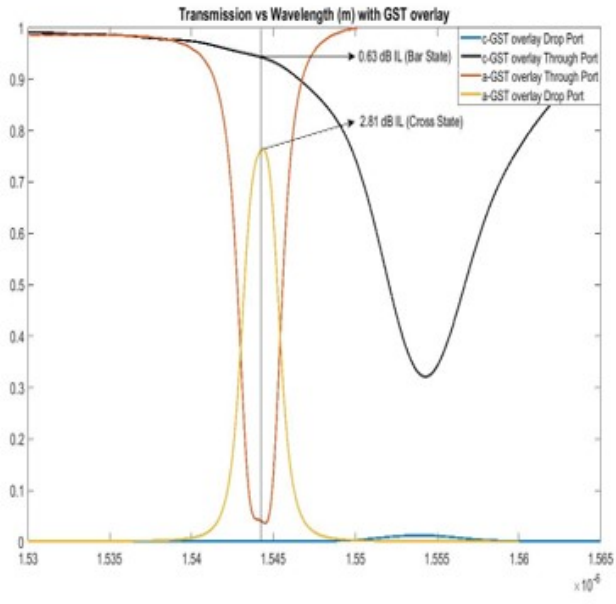

(a)

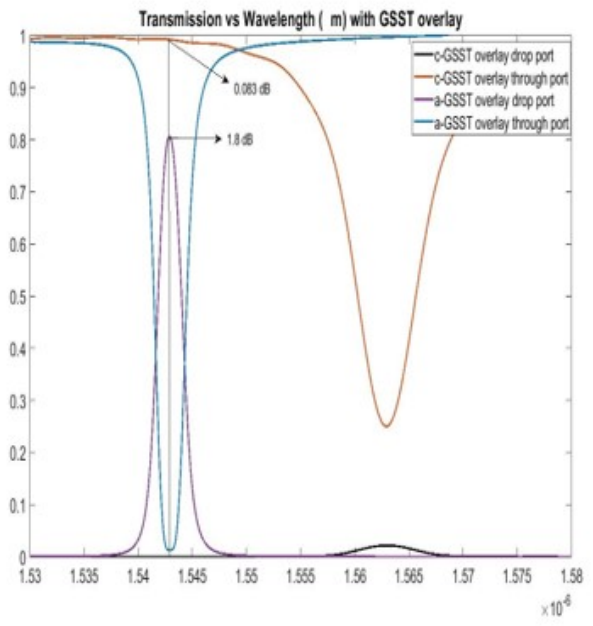

(b)

Figure 4.8: Transmission response with (a) GST and (b) GSST overlays for the device proposed in 4.4

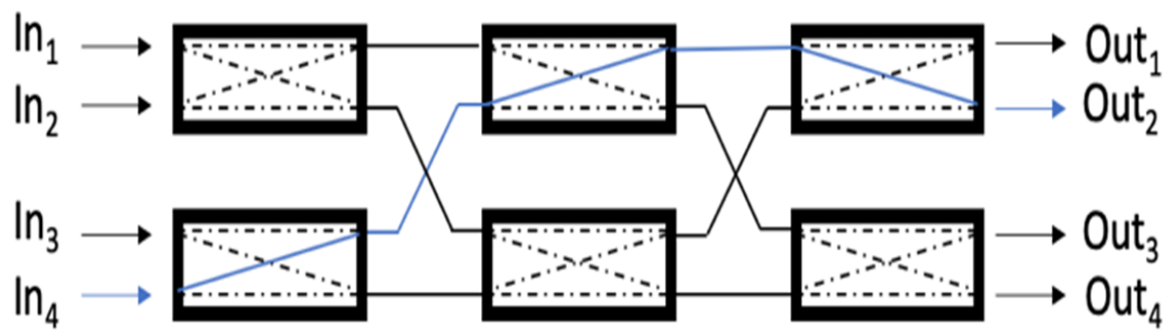

Figure 4.9: Schematic of a possible $2^{m} \times 2^{m}(\mathrm{~m}=2)$ Benes network switch. The blue path indicates a possible all-cross state.

Generally, a $2 \mathrm{~m} \times 2 \mathrm{~m}$ switch consists of $(2 \mathrm{~m}-1)$ rows and $(2 \mathrm{~m}-1)$ columns of $2 \times 2$ switches. Therefore, light always passes through a total of $(2 \mathrm{~m}-1) 2 \times 2$ switches in the fabric. To compute the IL of the entire network, we consider ILs from individual 2x2 switches on the optical path as well as loss due to waveguide crossings. The IL of a waveguide is taken as $0.1 \mathrm{~dB}$, which has been experimentally realized in the C-band. The ILs of the all-bar and all-cross states approximately correspond to the upper and lower bounds of the network IL. For all-bar state, IL will be: 


$$
\left(2^{m}-2\right) \times 0.1 d B+(2 m-1) \times I L_{b a r}
$$

Also, for an all-cross state path (highlighted blue in Fig 7) the corresponding IL is:

$$
\left(3 \times 2^{m}-1-2 m\right) \times 0.1 d B+(2 m-1) \times I L_{\text {cross }}
$$

Fig. 4.10 shows the switching performance of our proposed device as a function of $\mathrm{m}$ in the Benes network switch, demonstrating the potential of the scalability of the switch for both types of overcladding. Table 4.1 provides a performance comparison of two types of

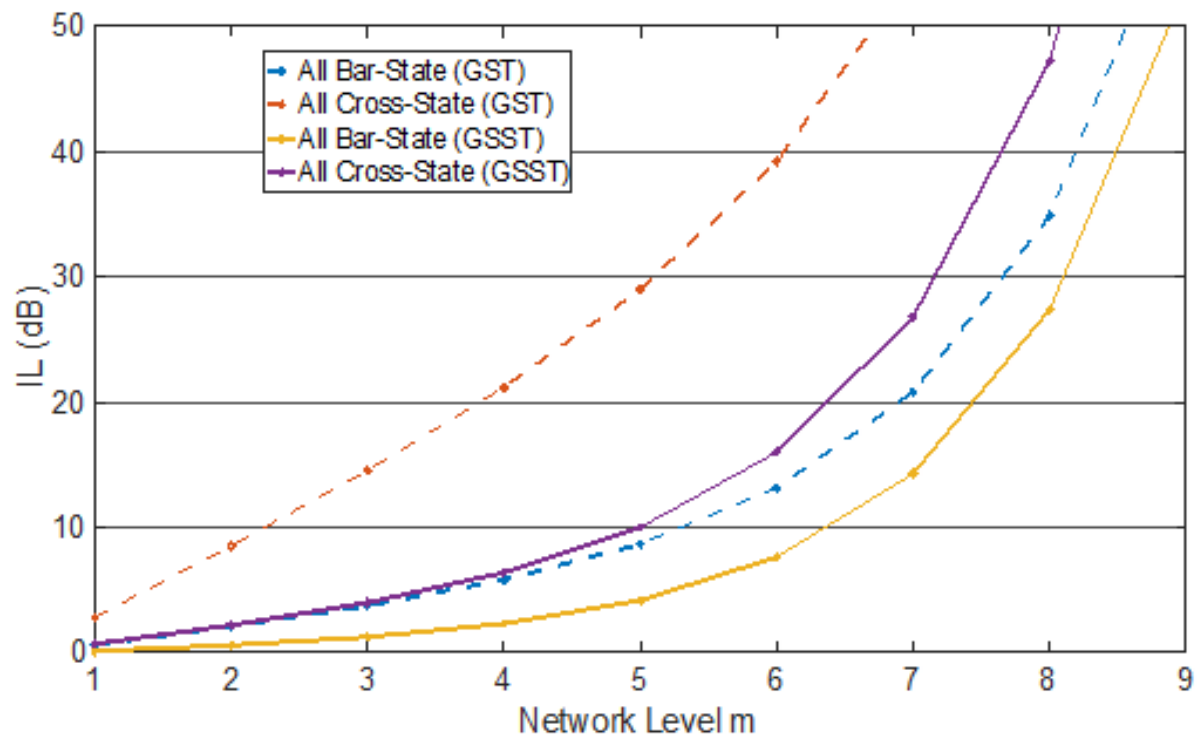

Figure 4.10: Insertion loss of our proposed switch network at $1554 \mathrm{~nm}$.

cladding for our proposed serially coupled ring resonator based 2x2 switch:

\subsection{Summary}

In this chapter, we proposed a double ring resonator based $2 \times 2$ switch with compact footprint. GSST based PCM has very low extinction coefficient. Thus offers low propagation 
Table 4.1: Performance Comparison of 2x2 switch with double ring resonators

\begin{tabular}{|c|l|l|l|l|}
\hline & $\begin{array}{l}\text { Bar-state } \\
(\mathrm{GST})\end{array}$ & $\begin{array}{l}\text { Cross- } \\
\text { state } \\
(\mathrm{GST})\end{array}$ & $\begin{array}{l}\text { Bar-state } \\
\text { (GSST) }\end{array}$ & $\begin{array}{l}\text { Cross- } \\
\text { state } \\
(\mathrm{GSST})\end{array}$ \\
\hline IL (dB) & 0.63 & 2.81 & 0.13 & 0.7 \\
\hline CT $(\mathrm{dB})$ & -28 & -13 & -38 & -23 \\
\hline
\end{tabular}

loss. We look at the scalability of $2^{m} \times 2^{m}$ Benes Network with our switch design. With our GSST overlay design, we predict overall insertion loss of a 32x32 Benes Network to be less than $20 \mathrm{~dB}$. We predict our design to be more compact and power efficient as compared a directional coupler switch at the cost of bandwidth and higher optical loss. For future work more fabrication tolerant design will be considered such as racetrack rings and micro-disks. 


\section{Chapter 5}

\section{Conclusion}

\subsection{Summary of Thesis}

This thesis covered the details of phase control in Photonic Integrated Circuits (PICs) and their applications. Optical phased arrays (OPA) an important PIC device for beamsteering and beamforming requires phase control of its antenna elements. In this thesis work, we present a control mechanism to drive down the phase errors of an OPA. We show that our control algorithm is a faster approach than traditional gradient descent based algorithms since it requires fewer function evaluations and smaller parameter space. We performed an experiment with an 8-element circular OPA and compared the experimental data with our simulations. We verified the frequency doubling effect with applied bias through simulations and experimentation. We also present a methodology to optimize the concentric ring based OPAs. We show that despite routing constraints due to the size of the grating couplers, we can achieve peak side lobe level performance of $-13.2 \mathrm{~dB}$ with $200 \mu m$ and $110 \mu m$ concentric rings. We also show that keeping the total number of elements invariant, we can achieve better side lobe and beam width performance with more rings in the system. We proposed a 4 ring configuration with $-15.3 \mathrm{~dB}$ side lobe performance with a beamwidth of $1^{\circ}$. 
We also looked at the phase control of PICs with relatively new class of phase change materials. Due to high modulation bandwidth phase change materials provide elegant solutions to some problems in optical computing. We propose a reconfigurable optical logic architecture using pn-modulation and optical memory in microdisk resonators. This is the first ever work that proposes the two modulation schemes in the same device. Reconfiguration signal in any logic operation requires large operation bandwidth. The phase change material is an excellent candidate to perform that operation while the pn-modulation can be used for fast optical logic processing. We show that our logic operations can be operated with low energy requirement (Max $10 \mu J)$ and high speed (15 $\mathrm{GHz})$.

Phase control in PIC can also be applied in optical routing. In this thesis, we propose non-volatile switching using phase change material as overlay. We introduce the concepts of wavelength-selective routing and spatial routing and show that we can design devices that take advantage of both techniques. We then propose a 2x2 photonic switch with coupled ring resonators. We show that by trading off the bandwidth of the switch we can design a very low loss and compact switching architecture.

\subsection{Statement of Impact}

Phased Array antenna has been a widely examined device in radio frequencies. Their ability to steer and direct beam without physically moving or altering the antenna has been useful in broadcasting applications, space communications, human-machine interfaces and others. Phased Arrays operating in radio frequencies have limitations in large scale integration. Optical Phased Arrays (OPAs) operating at short wavelengths have the potential for large scale integration with cost-effective approaches. We collaborated with Honeywell Aerospace to optimize the design and in-lab characterization of ElectroOptical Phased Arrays. Electronically steerable optical phased arrays would eliminate the need for complex pointing mechanisms with reduced mass, size and a more reliable oper- 
ation. Our work on optimizing the location of emitters through far field simulations has been important in designing the OPA chip that satisfies the required performance metrics. We also worked on setting up the test bench to characterize the OPA chip. Our control algorithm developed through simulations and experimental results can be useful in steering the collimated beam generated from the OPA. This work has already been adopted at Honeywell Aerospace and we are currently working toward a patent application.

Optical routing and computing are important disciplines in photonic integrated circuits. To the best of our knowledge we proposed the first dual ring based $2 \times 2$ non-volatile photonic switch. Our design was presented in Photonics North 2020 an international conference based in Canada. We also proposed a reconfigurable optical logic architecture that operates with carrier depletion and optical memory in microdisk modulators. This logic device retains the high speed operation of carrier depletion while maintains a wide modulation bandwidth for the recofigurable signal through optical memory. We are currently preparing a manuscript to publish our findings.

The following article has been accepted for publication as a result of the work done for this thesis:

M. A. R. Fatin and W. N. Ye, "Non-volatile Switching for Wavelength-Selective Spatial Routing", submitted to Photonics North, Niagara Falls, Canada, May 2020 (Accepted)

\subsection{Future Works}

\subsubsection{Improving Insertion Loss Performance}

In chapter 4 we proposed a coupled ring resonator based $2 \times 2$ switch with insertion loss performance of less than $20 \mathrm{~dB}$ for a 32x32 Benes network switch. We can improve our performances by using wrapped waveguide microdisks (Figure 5.1) rather than rings since they provide better coupling. Using racetrack resonators instead of rings should also provide better performance. 


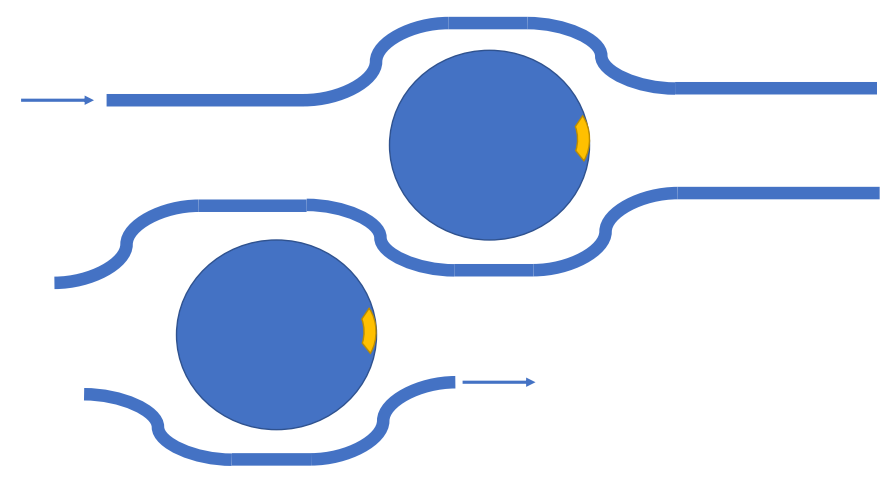

Figure 5.1: Wrapped waveguide dual microdisk switches

\subsubsection{Full demonstration of our proposed control algorithm}

Demonstrating beam steering in an 8 emitter OPA is challenging task owing to high peak sidelobes and low steering range. For future work, full demonstration of the control algorithm with a 100 emitter OPA in telecommunication wavelength can be greatly beneficial.

\subsubsection{Implementing Reconfigurable Switches on a Photonic Neural Net- work Architecture}

Physical realization of Neural Networks in a photonics platform is an exciting field in our era. An artificial neural network requires both linear and non-linear functions where the linear function implements the linear synaptic operation and the nonlinear function is used to sum the weighted inputs [15]. A variety of approaches are available for generating nonlinearity in photonic NNs. We propose a device concept similar to one shown in Fig. 5.2 that can be used to generate a nonlinear response function. The key idea behind our approach is that we can utilize the intermediary states between amorphous and crystalline states by applying varying light pulses on the GST sample and thus can be quasi-continuously tuned. We simulated for the device presented in figure 4.6 for the 


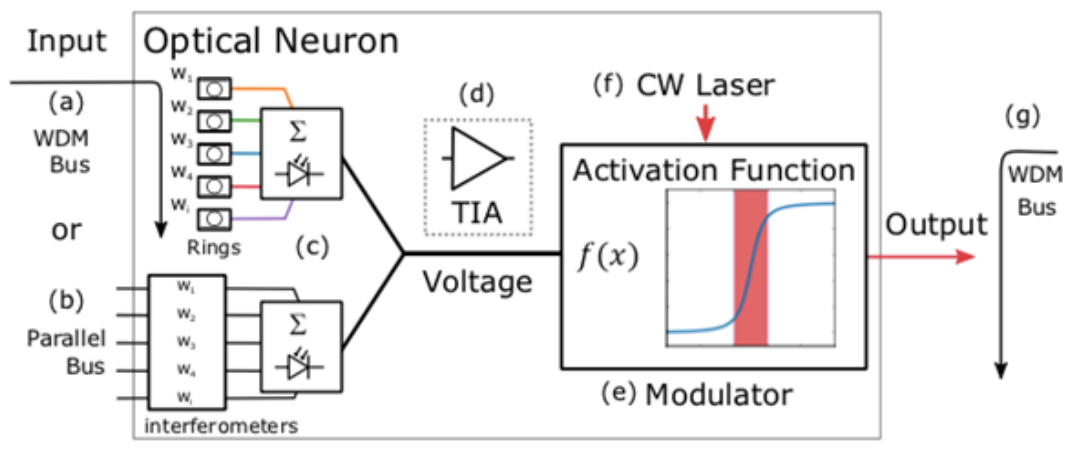

Figure 5.2: An electro-optic neuron concept taken from [15]. They operate taking input from a WDM bus relayed through a set of ring resonators or from a parallel bus containing optical interferometers then converted to an electrical signal from optical signal with a photodiode. The signal then amplified by a TIA modulates a CW laser that produces a non-linear transfer function at the output.

PCM material under different degrees of crystallization using Lumerical's 3D FDTD. Figure 5.3 shows the nonlinear response generated from our device with different degrees of crystallization. For future work, we have the potential to use this nonlinearity to physically realize artificial neural networks. Thus, we can use phase change materials to open a new avenue in the application of photonic neural networks.

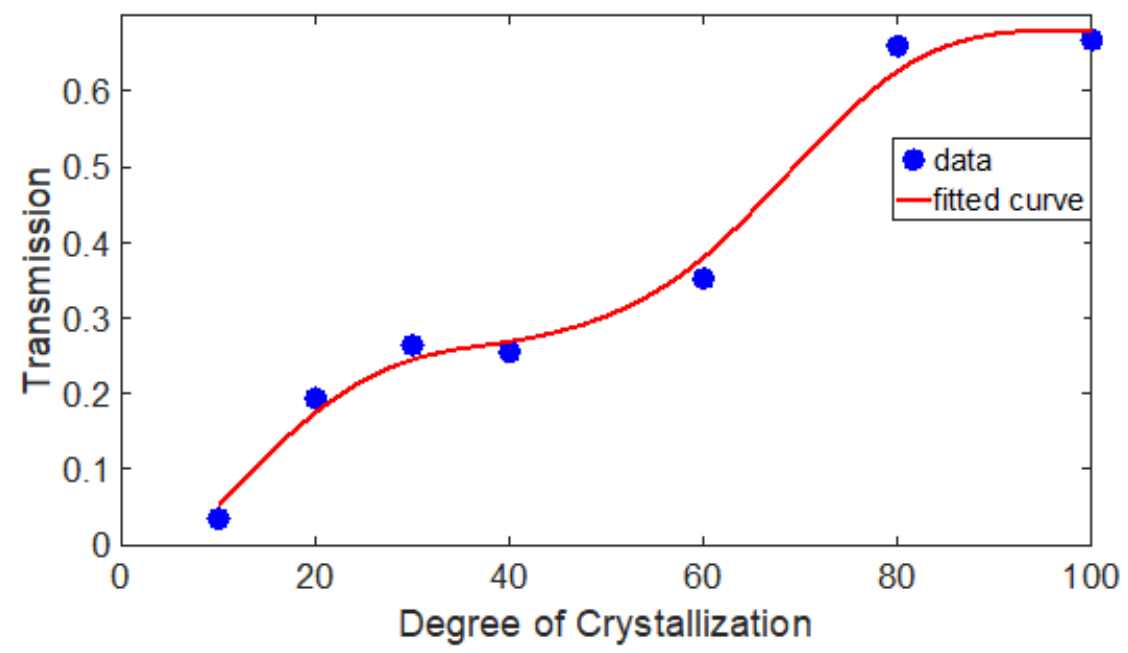

Figure 5.3: Gaussian fit of simulated data points for the device presented in Fig. 4.6. 


\section{Bibliography}

[1] Aflatouni, F., Abiri, B., Rekhi, A., And Hajimiri, A. Nanophotonic projection system. Optics express 23, 16 (2015), 21012-21022.

[2] Bergman, K. Silicon photonics for high performance interconnection networks. In Optical Fiber Communication Conference (2018), Optical Society of America, pp. Tu3F1.

[3] Bergman, K., Carloni, L. P., Biberman, A., Chan, J., And Hendry, G. Photonic network-on-chip design. Springer, 2014.

[4] Bogaerts, W., Pérez, D., Capmany, J., Miller, D. A., Poon, J., Englund, D., Morichetti, F., And Melloni, A. Programmable photonic circuits. Nature 586, 7828 (2020), 207-216.

[5] CaO, T., Fei, Y., Zhang, L., CaO, Y., And Chen, S. Design of a silicon machzehnder modulator with a u-type pn junction. Applied optics 52, 24 (2013), 5941-5948.

[6] Chakraborty, I., SAHa, G., AND Roy, K. Photonic in-memory computing primitive for spiking neural networks using phase-change materials. Physical Review Applied 11, 1 (2019), 014063.

[7] Chakraborty, I., Saha, G., Sengupta, A., And Roy, K. Toward fast neural computing using all-photonic phase change spiking neurons. Scientific reports 8,1 (2018), 1-9. 
[8] Chan, J., and Bergman, K. Photonic interconnection network architectures using wavelength-selective spatial routing for chip-scale communications. IEEE/OSA Journal of Optical communications and networking 4, 3 (2012), 189-201.

[9] Chan, J., Hendry, G., Biberman, A., and Bergman, K. Architectural exploration of chip-scale photonic interconnection network designs using physical-layer analysis. Journal of Lightwave Technology 28, 9 (2010), 1305-1315.

[10] Chan, J., Ophir, N., Lai, C. P., Biberman, A., Lira, H. L., Lipson, M., And BERGMAN, K. Data transmission using wavelength-selective spatial routing for photonic interconnection networks. In 2011 Optical Fiber Communication Conference and Exposition and the National Fiber Optic Engineers Conference (2011), IEEE, pp. 1-3.

[11] Chen, Y., Li, X., Sonnefraud, Y., FernándeZ-Domínguez, A. I., Luo, X., HONG, M., AND MAIER, S. A. Engineering the phase front of light with phasechange material based planar lenses. Scientific reports 5, 1 (2015), 1-7.

[12] Chrostowski, L., Grist, S., Flueckiger, J., Shi, W., Wang, X., Ouellet, E., Yun, H., Webb, M., Nie, B., LiAnG, Z., ET Al. Silicon photonic resonator sensors and devices. In Laser Resonators, Microresonators, and Beam Control XIV (2012), vol. 8236, International Society for Optics and Photonics, p. 823620.

[13] Chrostowski, L., AND HochberG, M. Silicon photonics design: from devices to systems. Cambridge University Press, 2015.

[14] Chu, C. H., Tseng, M. L., Chen, J., Wu, P. C., Chen, Y.-H., Wang, H.-C., Chen, T.-Y., Hsieh, W. T., WU, H. J., SUN, G., ET AL. Active dielectric metasurface based on phase-change medium. Laser \& Photonics Reviews 10, 6 (2016), 986-994.

[15] George, J. K., Mehrabian, A., Amin, R., Meng, J., De Lima, T. F., Tait, A. N., Shastri, B. J., El-Ghazawi, T., Prucnal, P. R., And Sorger, V. J. Neuromorphic photonics with electro-absorption modulators. Optics express 27, 4 (2019), 51815191. 
[16] Goodman, J. W. Introduction to Fourier optics. Roberts and Company Publishers, 2005.

[17] Gostimirovic, D., AND Winnie, N. Y. Ultracompact cmos-compatible optical logic using carrier depletion in microdisk resonators. Scientific Reports 7, 1 (2017), $1-10$.

[18] Guo, E.-J., Guo, H., Lu, H., Jin, K., HE, M., AND YANG, G. Structure and characteristics of ultrathin indium tin oxide films. Applied Physics Letters 98, 1 (2011), 011905.

[19] Harris, N. C., Carolan, J., Bunandar, D., Prabhu, M., Hochberg, M., Baehr-Jones, T., Fanto, M. L., Smith, A. M., Tison, C. C., Alsing, P. M., ET AL. Linear programmable nanophotonic processors. Optica 5, 12 (2018), 16231631.

[20] Hum, S. V. Radio and microwave wireless systems. https://www.waves.utoronto.ca/prof/svhum/ece422.html.

[21] Hutchison, D. N., Sun, J., Doylend, J. K., Kumar, R., Heck, J., Kim, W., Phare, C. T., Feshali, A., AND Rong, H. High-resolution aliasing-free optical beam steering. Optica 3, 8 (2016), 887-890.

[22] Khope, A. S., Saeidi, M., Yu, R., Wu, X., Netherton, A. M., LiU, Y., Zhang, Z., XiA, Y., Fleeman, G., SPOtT, A., ET AL. Multi-wavelength selective crossbar switch. Optics express 27, 4 (2019), 5203-5216.

[23] Miscuglio, M., Meng, J., Yesiliurt, O., Zhang, Y., Prokopeva, L. J., MehraBian, A., Hu, J., Kildishev, A. V., And Sorger, V. J. Artificial synapse with mnemonic functionality using gsst-based photonic integrated memory. In 2020 International Applied Computational Electromagnetics Society Symposium (ACES) (2020), IEEE, pp. 1-3. 
[24] RaOuX, S., XiOng, F., Wuttig, M., AND PoP, E. Phase change materials and phase change memory. MRS bulletin 39, 8 (2014), 703-710.

[25] Reed, G. T., AND KNIGHTS, A. P. Silicon photonics: an introduction. John Wiley \& Sons, 2004.

[26] Ríos, C., Stegmaier, M., Hosseini, P., Wang, D., Scherer, T., Wright, C. D., Bhaskaran, H., AND Pernice, W. H. Integrated all-photonic non-volatile multilevel memory. Nature Photonics 9, 11 (2015), 725-732.

[27] Sethi, P., AND Roy, S. All-optical ultrafast switching in $2 \times 2$ silicon microring resonators and its application to reconfigurable demux/mux and reversible logic gates. Journal of lightwave technology 32, 12 (2014), 2173-2180.

[28] Shen, Y., Harris, N. C., Skirlo, S., Prabhu, M., Baehr-Jones, T., HochberG, M., Sun, X., Zhao, S., Larochelle, H., Englund, D., ET Al. Deep learning with coherent nanophotonic circuits. Nature Photonics 11, 7 (2017), 441.

[29] Soref, R. A., AND Little, B. E. Proposed n-wavelength m-fiber wdm crossconnect switch using active microring resonators. IEEE Photonics Technology Letters 10, 8 (1998), 1121-1123.

[30] Sun, J., Kumar, R., SAKib, M., Driscoll, J. B., JayATilleka, H., AND Rong, H. A $128 \mathrm{gb} / \mathrm{s}$ pam4 silicon microring modulator with integrated thermo-optic resonance tuning. Journal of Lightwave Technology 37, 1 (2019), 110-115.

[31] TREES, H. Optimum array processing (detection, estimation, and modulation theory, part iv) wiley, 2002.

[32] Wang, J., Paesani, S., Ding, Y., Santagati, R., Skrzypczyk, P., Salavrakos, A., Tura, J., Augusiak, R., MANČInSKa, L., BACCO, D., ET Al. Multidimensional quantum entanglement with large-scale integrated optics. Science 360, 6386 (2018), 285-291. 
[33] WANG, L., Wen, J., YANG, C., AND XiONG, B. Potential of ito thin film for electrical probe memory applications. Science and technology of advanced materials 19, 1 (2018), 791-801.

[34] Xie, W., Komljenovic, T., Huang, J., Tran, M., Davenport, M., Torres, A., Pintus, P., AND Bowers, J. Heterogeneous silicon photonics sensing for autonomous cars. Optics express 27, 3 (2019), 3642-3663.

[35] Zhang, Q., Zhang, Y., LI, J., SORef, R., GU, T., AND HU, J. Broadband nonvolatile photonic switching based on optical phase change materials: beyond the classical figure-of-merit. Optics letters 43, 1 (2018), 94-97.

[36] Zhang, Y., Chou, J. B., Li, J., Li, H., Du, Q., YADAV, A., Zhou, S., Shalaginov, M. Y., FANG, Z., ZHONG, H., ET AL. Broadband transparent optical phase change materials for high-performance nonvolatile photonics. Nature communications 10, 1 (2019), 1-9.

[37] Zheng, J., Khanolkar, A., Xu, P., Colburn, S., Deshmukh, S., Myers, J., Frantz, J., Pop, E., Hendrickson, J., Doylend, J., ET Al. Gst-on-silicon hybrid nanophotonic integrated circuits: a non-volatile quasi-continuously reprogrammable platform. Optical Materials Express 8, 6 (2018), 1551-1561. 